\title{
A FUNCTIONAL CENTRAL LIMIT THEOREM FOR SPATIAL BIRTH AND DEATH PROCESSES
}

\author{
XIN QI,* University of Wisconsin-Madison
}

\begin{abstract}
We give a functional central limit theorem for spatial birth and death processes based on the representation of such processes as solutions of stochastic equations. For any bounded and integrable function in Euclidean space, we define a family of processes which is obtained by integrals of this function with respect to the centered and scaled spatial birth and death process with constant death rate. We prove that this family converges weakly to a Gaussian process as the scale parameter goes to infinity. We do not need the birth rates to have a finite range of interaction. Instead, we require that the birth rates have a range of interaction that decays polynomially. In order to show the convergence of the finitedimensional distributions of the above processes, we extend Penrose's multivariate spatial central limit theorem. An example of the asymptotic normalities of the time-invariance estimators for the birth rates of spatial point processes is given.
\end{abstract}

Keywords: Spatial birth and death process; Poisson random measure; stochastic equation; functional central limit theorem

2000 Mathematics Subject Classification: Primary 60G55

Secondary 60F $17 ; 60 \mathrm{H} 20 ; 60 \mathrm{~K} 35$

\section{Introduction}

Central limit theorems (CLTs) for interacting particle systems have a long history. Holley and Stroock (1979) investigated large-scale behavior of certain classes of interacting particle systems on $\mathbb{Z}^{d}$ and proved that, upon being averaged over increasingly large blocks of sites, these processes converge weakly to Gaussian processes. Kesten and Lee (1996) proved a CLT for weighted minimal spanning trees on random points. They applied a martingale CLT to a sum of martingale differences. One of the key points in their approach was the use of the monotonicity property for minimal spanning trees, which was extended to the stabilization property in Lee (1997). The stabilization property states that if a homogeneous rate 1 Poisson process is constructed by successively adding Poisson points in annuli centered at the origin then the local structure of the successive minimal spanning trees eventually stabilizes. The martingale method and the stabilization property were developed to give a CLT for functionals of lattice-indexed white noise in Penrose (2001). Let $X=\left(X_{x}, x \in \mathbb{Z}^{d}\right)$ be a family of independent, identically distributed white noises. Penrose considered a family of random variables $\{H(X, B): B$ over all the finite subsets of integer points $\}$. For each finite subset $B$ of $\mathbb{Z}^{d}, H(X, B)$ is a function of $\left(X_{x}, x \in B\right)$. He gave the CLT for $H(X, B)$ as the set $B$ became large. Roughly speaking, his stabilization condition is that the effect on the functionals of changing the value of the lattice system at a single site is local. Penrose (2005) established a multivariate CLT for a general lattice system. The basic setting was similar to Penrose (2001),

Received 30 July 2007; revision received 15 May 2008.

* Current address: 1199 Whitney Avenue, Apt. 507, Hamden, CT 06517, USA. Email address: xinqi@ stat.wisc.edu

Supported in part by the National Science Foundation under grant DMS 05-03983. 
but $X_{x}$ was not assumed to be Gaussian, and $B$ was over all bounded subsets in $\mathbb{R}^{d}$ with zero Lebesgue measure boundary. A novel feature of Penrose (2005) is that one more stabilization condition was added. However, he used the assumption that the random variables $H(X, B)$ depend only on a finite collection of $X_{x}$. Since we are mainly concerned with functionals of spatial birth and death processes, which do not satisfy the assumption, we prove a multivariate CLT without it by adding the third stabilization condition.

In this paper we consider spatial birth and death processes in $\mathbb{R}^{d}$, where $d$ is a positive integer. We identify the countable subset of points in $\mathbb{R}^{d}$ with the counting measure $\eta$ given by assigning unit mass to each point, that is, $\eta(B)$ denotes the number of points in a set $B \in \mathcal{B}\left(\mathbb{R}^{d}\right)$ (where $\mathscr{B}\left(\mathbb{R}^{d}\right)$ denotes the Borel subsets of $\mathbb{R}^{d}$ ). In Garcia and Kurtz (2006) spatial birth and death processes were obtained as solutions of a system of stochastic equations. The processes are required to be locally finite, but may involve an infinite population over the full (noncompact) type space. A key point in this paper is that we apply our CLT to the stochastic equations of Garcia and Kurtz (2006) to obtain functional CLTs for integrals of all bounded and integrable functions with respect to the spatial birth and death processes.

After having completed this paper, we noticed the recent paper of Penrose (2007). There is an overlap between these two papers. Penrose considered more general processes and also handled processes with nonconstant death rate and even with immigration. However, in Penrose (2007) the assumption of finite-range interaction was essential, which is used to prove the existence of the Markov processes and to prove the CLT. In this paper we do not require finite-range interaction, instead we assume a polynomial rate of decay of dependence.

The two main theorems are stated in Section 2 and their proofs are given in Sections 3 and 4. In Section 5 we present an example of the asymptotic normalities of the time-invariance estimators for the birth rates of spatial point processes.

\section{Definitions and main results}

First we introduce some notation from Penrose (2005) which we will use throughout this paper. For $x=\left(x_{1}, \ldots, x_{d}\right) \in \mathbb{R}^{d}$, write $|x|$ for the Euclidean norm of $x$ and $\|x\|:=$ $\max _{1 \leq i \leq d}\left|x_{i}\right|$. For $A \subseteq \mathbb{R}^{d}, l \in \mathbb{R}$, and $y \in \mathbb{R}^{d}$, let $l A$ denote the scaled set $\{l x: x \in A\}$, and let $\tau_{y}(A)$ denote the translated set $\{y+x: x \in A\}$. Let $\partial(A)$ denote the boundary of $A$. If $A$ is Lebesgue measurable, write $|A|$ for its Lebesgue measure. Let $\tilde{A}$ denote the discretization of $A$ given by $\tilde{A}:=\left\{z \in \mathbb{Z}^{d}: B_{\rho}(z) \cap A \neq \varnothing\right\}$, where $\rho \geq \sqrt{d}$ is a constant and $B_{\rho}(z)$ denotes the open ball with center $z$ and radius $\rho$.

Let $\mathcal{R}\left(\mathbb{R}^{d}\right)$ denote the collection of Riemann measurable subsets (bounded measurable subsets of $\mathbb{R}^{d}$ with zero Lebesgue boundary) of $\mathbb{R}^{d}$. Let

$$
X=\left(X_{z}, z \in \mathbb{Z}^{d}\right)
$$

be a family of independent, identically distributed random elements, and let

$$
H=\left\{H_{l}(X, A): l \geq 1, A \in \mathcal{R}\left(\mathbb{R}^{d}\right)\right\}
$$

be a collection of random variables where, for each $l \geq 1$ and $A \in \mathcal{R}\left(\mathbb{R}^{d}\right), H_{l}(X, A)$ is a function of $\left(X_{z}, z \in \mathbb{Z}^{d}\right)$. We call $H$ a random set function. We write $H_{l}(X, A)$ as $H_{l}(A)$ whenever there is no possibility of confusion. For example, in this paper $H_{l}(A)=\eta_{t}(l A)$. We are interested in the weak convergence to a normal distribution of the family

$$
l^{-d / 2}\left(H_{l}(A)-\mathrm{E}\left[H_{l}(A)\right]\right), \quad l \geq 1 .
$$


Penrose (2005) considered the case in which $H_{l}(A)$ was a function of $\left(X_{z}, z \in \widetilde{l C_{0}}\right)$, where $C_{0}$ is a Riemann measurable subset of $\mathbb{R}^{d}$. Hence, $H_{l}(A)$ depends only on a finite collection of elements in $\left(X_{z}, z \in \mathbb{Z}^{d}\right)$. But the spatial birth and death processes in this paper do not satisfy this condition. Hence, we extend the CLT to the case in which $H_{l}(A)$ depends on the whole system. We add one more condition to the conditions given in Penrose (2005).

Let $X_{*}$ be a copy of $X_{o}$ and independent of the family $X$, where $o$ is the origin of $\mathbb{Z}^{d}$. Let $H=\left\{H_{l}(A)=H_{l}(X, A): l \geq 1, A \in \mathcal{R}\left(\mathbb{R}^{d}\right)\right\}$ be a random set function. For $y, z \in \mathbb{Z}^{d}$, write $y \prec z$ if $y$ precedes $z$ in the lexicographic ordering on $\mathbb{Z}^{d}$, and write $y \preccurlyeq z$ if either $y \prec z$ or $y=z$. Let $\mathcal{F}_{y}=\bigvee_{z \in \mathbb{Z}^{d}, z \preccurlyeq y} \sigma\left\{X_{z}\right\}$. For $l \geq 1$ and $y \in \mathbb{Z}^{d}$, define $H_{l, y}(A):=H_{l}\left(\tau_{y} X, A\right)$, where $\tau_{y} X$ denotes the shifted family $\left(X_{z+y}, z \in \mathbb{Z}^{d}\right)$. For any $y \in \mathbb{Z}^{d}$, define $X^{y}$ to be the family $X$ with the value $X_{y}$ at $y$ replaced by the independent copy $X_{*}$, but with the values at all the other sites the same. Let $\Delta_{l, y}^{H}(A)=H_{l, y}(X, A)-H_{l, y}\left(X^{o}, A\right)$.

Condition 2.1. (Stabilization conditions.) There exists a random variable $\Delta_{\infty}^{H}$ such that, for any sequence $\left\{\left(l_{n}, y_{n}\right) \mid l_{n} \geq 1, y_{n} \in \mathbb{Z}^{d}\right\}$ and any $A \in \mathcal{R}\left(\mathbb{R}^{d}\right)$,

$$
\begin{aligned}
& \Delta_{l_{n}, y_{n}}^{H}(A) \stackrel{\mathrm{P}}{\rightarrow} \Delta_{\infty}^{H} \text { if } \liminf _{n \rightarrow \infty}\left(\tau_{y_{n}}\left(l_{n} A\right)\right)=\mathbb{R}^{d}, \\
& \Delta_{l_{n}, y_{n}}^{H}(A) \stackrel{\mathrm{P}}{\rightarrow} 0 \text { if } \quad \liminf _{n \rightarrow \infty}\left(\tau_{y_{n}}\left(l_{n}\left(A^{\mathrm{c}}\right)\right)\right)=\mathbb{R}^{d},
\end{aligned}
$$

where $\stackrel{\text { P }}{\rightarrow}$ 'denotes convergence in probability, and, for each $A \in \mathcal{R}\left(\mathbb{R}^{d}\right)$, there exists $K_{A}>0$ (depending on A) such that

$$
\lim _{l \rightarrow \infty} \frac{1}{l^{d}} \sum_{\substack{y \in \mathbb{Z}^{d} \\\|y\| \geq l K_{A}}} \mathrm{E}\left[\left(\Delta_{l, y}^{H}(A)\right)^{2}\right]=0 .
$$

Condition 2.2. (Moment condition.) There exists $\gamma>2$ such that

$$
\sup \left\{\mathrm{E}\left[\left|\Delta_{l,-y}^{H}(A)\right|^{\gamma}\right]: A \in \mathcal{R}\left(\mathbb{R}^{d}\right), l \geq 1, y \in \mathbb{Z}^{d}\right\}<\infty .
$$

Theorem 2.1. Suppose that $H^{1}, \ldots, H^{k}$ are random set functions and are integrable for each $l \geq 1$ and $A \in \mathcal{R}\left(\mathbb{R}^{d}\right)$. Each of them satisfies Conditions 2.1 and 2.2 for some $\gamma>2$. Let the $k \times k$ matrix $\left(\sigma_{i j}^{*}\right)_{i, j=1}^{k}$ be given by

$$
\sigma_{i, j}^{*}:=\mathrm{E}\left[\mathrm{E}\left[\Delta_{\infty}^{H^{i}} \mid \mathcal{F}_{o}\right] \mathrm{E}\left[\Delta_{\infty}^{H^{j}} \mid \mathcal{F}_{o}\right]\right],
$$

where $o$ is the origin in $\mathbb{Z}^{d}$. Then, if $A_{1}, \ldots, A_{k} \in \mathcal{R}\left(\mathbb{R}^{d}\right)$, we have

$$
\lim _{l \rightarrow \infty} l^{-d} \operatorname{cov}\left(H_{l}^{i}\left(A_{i}\right), H_{l}^{j}\left(A_{j}\right)\right)=\sigma_{i, j}^{*}\left|A_{i} \cap A_{j}\right|,
$$

and, as $l \rightarrow \infty$,

$$
\left(l^{-d / 2}\left(H_{l}^{i}\left(A_{i}\right)-\mathrm{E}\left[H_{l}^{i}\left(A_{i}\right)\right]\right)\right)_{i=1}^{k} \stackrel{\mathrm{D}}{\rightarrow} \mathcal{N}\left(o, \sigma_{i, j}^{*}\left|A_{i} \cap A_{j}\right|\right)_{i, j=1}^{k},
$$

where $\stackrel{\mathrm{D}}{\rightarrow}$ ' denotes convergence in distribution.

Remarks 2.1. (a) In Penrose (2005), Condition 2.1 contained only (2.1) and (2.2). However, the $H_{l}(A)$ were required to depend only on a finite number of random elements in the family $\left(X_{z}, z \in \mathbb{Z}^{d}\right)$. We do not require this restriction here. 
(b) Suppose that $H$ satisfies, for every $l \geq 1, y \in \mathbb{R}^{d}$, and $A \in \mathcal{R}\left(\mathbb{R}^{d}\right), H_{l}(X, A)=F(X, l A)$, where $\left\{F(X, A): A \in \mathcal{R}\left(\mathbb{R}^{d}\right)\right\}$ is a collection of random variables, each of which is a function of $X$. If $F$ is translation invariant, that is, $F\left(\tau_{y}(X), A\right)=F\left(X, \tau_{y}(A)\right)$, then the following conditions imply (2.1) and (2.2). There exists a random variable $\Delta_{\infty}^{H}$ such that, for any sequence $\left\{A_{n} \in \mathcal{R}\left(\mathbb{R}^{d}\right): n=1,2, \ldots\right\}$,

$$
\begin{gathered}
F\left(X, A_{n}\right)-F\left(X^{o}, A_{n}\right) \stackrel{\mathrm{P}}{\rightarrow} \Delta_{\infty}^{H} \quad \text { if } \quad \liminf _{n \rightarrow \infty}\left(A_{n}\right)=\mathbb{R}^{d}, \\
F\left(X, A_{n}\right)-F\left(X^{o}, A_{n}\right) \stackrel{\mathrm{P}}{\rightarrow} 0 \text { if } \quad \liminf _{n \rightarrow \infty}\left(A_{n}^{\mathrm{c}}\right)=\mathbb{R}^{d} .
\end{gathered}
$$

The result follows from the observation that

$$
\Delta_{l, y}^{H}(A)=H_{l, y}(X, A)-H_{l, y}\left(X^{o}, A\right)=F\left(X, \tau_{y}(l A)\right)-F\left(X^{0}, \tau_{y}(l A)\right) .
$$

In this paper we take $H_{l}(A)=\eta_{t}(l A)$, which satisfies the above conditions.

Let $\mathcal{N}\left(\mathbb{R}^{d}\right)$ be the collection of counting measures on $\mathbb{R}^{d}$. The state space of the spatial birth and death processes will be some subset of $\mathcal{N}\left(\mathbb{R}^{d}\right)$. All processes and random variables are defined on a complete probability space $(\Omega, \mathcal{F}, \mathrm{P})$. The spatial birth and death process is specified in terms of nonnegative functions $\lambda: \mathbb{R}^{d} \times \mathcal{N}\left(\mathbb{R}^{d}\right) \rightarrow[0,+\infty)$ and $b: \mathbb{R}^{d} \times \mathcal{N}\left(\mathbb{R}^{d}\right) \rightarrow$ $[0,+\infty)$. Here $\lambda(x, \eta)$ denotes the birth rate and $b(x, \eta)$ denotes the death rate. If the point configuration at time $t$ is $\eta \in \mathcal{N}\left(\mathbb{R}^{d}\right)$ then the probability that a point in a set $A \subset \mathbb{R}^{d}$ is added to the configuration in the next time interval of length $\Delta t$ is approximately $\int_{A} \lambda(x, \eta) \mathrm{d} x \Delta t$, and the probability that a point $x \in \eta$ is deleted from the configuration in the next time interval of length $\Delta t$ is approximately $b(x, \eta) \Delta t$. Under these assumptions, the generator of the process should be of the form

$$
\mathcal{A} f(\eta)=\int_{\mathbb{R}^{d}}\left(f\left(\eta+\delta_{x}\right)-f(\eta)\right) \lambda(x, \eta) \mathrm{d} u+\int_{\mathbb{R}^{d}}\left(f\left(\eta-\delta_{x}\right)-f(\eta)\right) b(x, \eta) \eta(\mathrm{d} x)
$$

for $f$ in an appropriate domain, where $\delta_{x}$ denotes the point set which contains only one point at $x$. Now we give our main theorem. For $l \geq 1$, define

$$
Y^{(l)}(f, t)=\frac{1}{l^{d / 2}}\left(\int_{\mathbb{R}^{d}} f\left(\frac{x}{l}\right) \eta_{t}(\mathrm{~d} x)-\mathrm{E}\left[\int_{\mathbb{R}^{d}} f\left(\frac{x}{l}\right) \eta_{t}(\mathrm{~d} x)\right]\right)
$$

for $t \geq 0$ and $f \in L^{1}\left(\mathbb{R}^{d}\right) \cap L^{\infty}\left(\mathbb{R}^{d}\right)$. Suppose that there exists a measurable function $a(x, y)$ on $\mathbb{R}^{\bar{d}} \times \mathbb{R}^{d}$ satisfying

$$
a(x, y) \geq \sup _{\eta}\left|\lambda\left(x, \eta+\delta_{y}\right)-\lambda(x, \eta)\right| .
$$

Theorem 2.2. Assume that the birth rate $\lambda$ is bounded by some positive number $L$ and that it is translation invariant. Suppose that $\eta_{0}$ is a Poisson random measure on $\mathbb{R}^{d}$ with constant intensity $\mu_{1}$. Suppose that

$$
a(x, y) \leq \frac{b}{1+|x-y|^{2 d+\delta}}
$$

for some constants $b>0$ and $\delta>0$. Assume that there exists a positive function $c(x)$ such that

$$
M=\sup _{x \in \mathbb{R}^{d}} \int_{\mathbb{R}^{d}} \frac{c(x) a(x, y)}{c(y)} \mathrm{d} y<\infty
$$


and that $c(x)$ is bounded in a neighborhood of the origin in $\mathbb{R}^{d}$, i.e. $\int_{\mathbb{R}^{d}}\left(1 / c(x)^{1 / 3}\right) \mathrm{d} x<\infty$. Then $Y^{(l)}$ converges to a Gaussian process $W$ indexed by $\left(L^{1}\left(\mathbb{R}^{d}\right) \cap L^{\infty}\left(\mathbb{R}^{d}\right)\right) \times[0, \infty)$ in the sense that, for any finite collection $\left\{f_{1}, \ldots, f_{n}\right\}$ of the functions in $L^{1}\left(\mathbb{R}^{d}\right) \cap L^{\infty}\left(\mathbb{R}^{d}\right)$,

$$
\left(Y^{(l)}\left(f_{1}, \cdot\right), \ldots, Y^{(l)}\left(f_{n}, \cdot\right)\right) \stackrel{\mathrm{D}}{\rightarrow}\left(W\left(f_{1}, \cdot\right), \ldots, W\left(f_{n}, \cdot\right)\right)
$$

as $l \rightarrow \infty$ in $D_{\mathbb{R}^{n}}[0, \infty)$. In addition, for each $f \in L^{1}\left(\mathbb{R}^{d}\right) \cap L^{\infty}\left(\mathbb{R}^{d}\right), W(f, \cdot)$ has continuous sample paths.

Remarks. (a) If inequality (2.5) is satisfied for some constant $\delta>d$, all the conditions on $c(x)$ can be derived from (2.5). In fact, we can take $c(x)=1+|x|^{2 d+\delta}$. Then $1 / c(x)^{1 / 3}$ is integrable and

$$
\begin{aligned}
M & =\sup _{x \in \mathbb{R}^{d}} \int_{\mathbb{R}^{d}} \frac{c(x) a(x, y)}{c(y)} \mathrm{d} y \\
& =\sup _{x \in \mathbb{R}^{d}} \int_{\mathbb{R}^{d}} \frac{\left(1+|x|^{2 d+\delta}\right) a(x, y)}{1+|y|^{2 d+\delta}} \mathrm{d} y \\
& =\sup _{x \in \mathbb{R}^{d}} \int_{\mathbb{R}^{d}} \frac{\left(1+|x-y+y|^{2 d+\delta}\right) a(x, y)}{1+|y|^{2 d+\delta}} \mathrm{d} y \\
& \leq \sup _{x \in \mathbb{R}^{d}} \int_{\mathbb{R}^{d}} \frac{b\left(1+2^{2 d+\delta-1}\left(|y|^{2 d+\delta}+|x-y|^{2 d+\delta}\right)\right)}{\left(1+|y|^{2 d+\delta}\right)\left(1+|x-y|^{2 d+\delta}\right)} \mathrm{d} y \\
& \leq \sup _{x \in \mathbb{R}^{d}} \int_{\mathbb{R}^{d}} \frac{2^{2 d+\delta-1} b\left(1+|y|^{2 d+\delta}+1+|x-y|^{2 d+\delta}\right)}{\left(1+|y|^{2 d+\delta}\right)\left(1+|x-y|^{2 d+\delta}\right)} \mathrm{d} y \\
& \leq \sup _{x \in \mathbb{R}^{d}} \int_{\mathbb{R}^{d}} \frac{2^{2 d+\delta-1} b}{1+|x-y|^{2 d+\delta}} \mathrm{d} y+\int_{\mathbb{R}^{d}} \frac{2^{2 d+\delta-1} b}{1+|y|^{2 d+\delta}} \mathrm{d} y \\
& \leq 2^{2 d+\delta} b \int_{\mathbb{R}^{d}} \frac{1}{1+|y|^{2 d+\delta}} \mathrm{d} y \\
& <\infty .
\end{aligned}
$$

If the birth rate has a finite-interaction range, that is, $a(x, y)=0$ if $|x-y|$ is bigger than some fixed constant which does not depend on $x$ and $y$, then it satisfies (2.5) for any $\delta>0$.

(b) If $c(x)$ is a positive function satisfying the conditions in Theorem 2.2 and $a(x, y)$ satisfies (2.5), then $c(x) \vee 1=\max \{c(x), 1\}$ also satisfies the conditions in Theorem 2.2. In fact, defining $\tilde{c}(x)=c(x) \vee 1$, we have

$$
\int_{\mathbb{R}^{d}} \frac{1}{\tilde{c}(x)^{1 / 3}} \mathrm{~d} x \leq \int_{\mathbb{R}^{d}} \frac{1}{c(x)^{1 / 3}} \mathrm{~d} x<\infty
$$

and

$$
\begin{aligned}
\sup _{x \in \mathbb{R}^{d}} \int_{\mathbb{R}^{d}} \frac{\tilde{c}(x) a(x, y)}{\tilde{c}(y)} \mathrm{d} y & \leq \sup _{x \in \mathbb{R}^{d}} \int_{\mathbb{R}^{d}} \frac{(c(x)+1) a(x, y)}{\tilde{c}(y)} \mathrm{d} y \\
& \leq \sup _{x \in \mathbb{R}^{d}} \int_{\mathbb{R}^{d}} \frac{c(x) a(x, y)}{\tilde{c}(y)} \mathrm{d} y+\sup _{x \in \mathbb{R}^{d}} \int_{\mathbb{R}^{d}} \frac{a(x, y)}{\tilde{c}(y)} \mathrm{d} y \\
& \leq \sup _{x \in \mathbb{R}^{d}} \int_{\mathbb{R}^{d}} \frac{c(x) a(x, y)}{c(y)} \mathrm{d} y+\sup _{x \in \mathbb{R}^{d}} \int_{\mathbb{R}^{d}} a(x, y) \mathrm{d} y
\end{aligned}
$$




$$
\begin{aligned}
& \leq \sup _{x \in \mathbb{R}^{d}} \int_{\mathbb{R}^{d}} \frac{c(x) a(x, y)}{c(y)} \mathrm{d} y+\int_{\mathbb{R}^{d}} \frac{b}{1+|y|^{2 d+\delta}} \mathrm{d} y \\
& <\infty
\end{aligned}
$$

Hence, we can choose $c(x)$ to be a function bounded from below by a positive number; so $1 / c(x)$ is bounded and, hence, $1 / c(x)^{\alpha}$ is integrable for all $\alpha \geq \frac{1}{3}$.

Let $E$ be the Schwartz space in $\mathbb{R}^{d}$. Let $E^{\prime}$ be its dual space.

Corollary 2.1. Suppose that the conditions of Theorem 2.2 are satisfied. Define a family of $E^{\prime}$-valued processes $\left\{\xi_{t}^{(l)}: t \geq 0, l \geq 1\right\}$. For any $f \in E$, the value of $\xi_{t}^{(l)}$ at $f$ is given by

$$
\xi_{t}^{(l)}(f)=\frac{1}{l^{d / 2}}\left(\int_{\mathbb{R}^{d}} f\left(\frac{x}{l}\right) \eta_{t}(\mathrm{~d} x)-\mathrm{E}\left[\int_{\mathbb{R}^{d}} f\left(\frac{x}{l}\right) \eta_{t}(\mathrm{~d} x)\right]\right) .
$$

Then the $\xi^{(l)}$ are $E^{\prime}$-valued processes with sample paths in $D_{E^{\prime}}[0, \infty)$ and there exists an $E^{\prime}$-valued process $\xi$ with sample paths in $C_{E^{\prime}}[0, \infty)$ such that $\xi^{(l)} \rightarrow \xi$ weakly.

Proof. The result follows immediately from Theorem 2.2 and Theorem 5.3 of Mitoma (1983).

\section{Proof of Theorem 2.1}

Let $\mathscr{H}_{k}=\bigvee_{y \in \mathbb{Z}^{d},\|y\| \geq k} \sigma\left\{X_{y}\right\}$, where $k$ is a nonnegative integer. Then $\mathscr{H}_{0}$ is the $\sigma$-field generated by all $\left(X_{z}\right)_{z \in \mathbb{Z}^{d}}$.

In Penrose (2005) the $H_{l}(A)$ were assumed to depend only on a finite collection of $X_{y}$; hence, $H_{l}(A)-\mathrm{E}\left[H_{l}(A)\right]$ could be written as a sum of finite martingale differences. In this paper we do not make this assumption. We write $H_{l}(A)-\mathrm{E}\left[H_{l}(A)\right]$ as the sum of $H_{l}(A)-\mathrm{E}\left[H_{l}(A) \mid \mathscr{H}_{\left[l K_{A}\right]}\right]$ and $\mathrm{E}\left[H_{l}(A) \mid \mathcal{H}_{\left[l K_{A}\right]}\right]-\mathrm{E}\left[H_{l}(A)\right]$, where $K_{A}$ is a fixed positive number and $\left[l K_{A}\right]$ denotes the largest integer number less than or equal to $l K_{A}$. The second terms will be shown to converge to 0 in $L^{2}(\mathrm{P})$ and the first terms can be written as a sum of finite martingale differences. Then we follow the steps of the proof of Theorem 2.1 of Penrose (2005) to show the weak convergence of the first terms. The following two technical lemmas are needed in our proof.

Lemma 3.1. Let $(\Omega, \mathcal{F}, \mathrm{P})$ be a probability space, and let $\left(\mathscr{D}_{n}, n \geq 0\right)$ be a decreasing sequence of sub- $\sigma$-fields of $\mathcal{F}$. If $\mathcal{E}$ is another sub- $\sigma$-field independent of $\mathscr{D}_{0}$ then

$$
\bigcap_{n}\left(\mathscr{E} \vee \mathscr{D}_{n}\right)=\mathscr{E} \vee \bigcap_{n} \mathscr{D}_{n}
$$

up to $\mathrm{P}$-negligible sets.

Proof. It is easy to see that $\varepsilon \vee \bigcap_{n} \mathscr{D}_{n} \subset \bigcap_{n}\left(\varepsilon \vee D_{n}\right)$. We prove the other direction. For any $C \in \mathscr{E}$ and $D \in \mathscr{D}_{0}$, define a bounded backward martingale $Y_{n}=\mathrm{E}\left[\mathbf{1}_{C \cap D} \mid \mathcal{E}\right.$ $\left.\vee D_{n}\right]=\mathbf{1}_{C} \mathrm{E}\left[\mathbf{1}_{D} \mid \mathscr{D}_{n}\right]$, where $\mathbf{1}_{C}$ is the indicator function of $C$. Since $Y_{n}$ converges to $\mathrm{E}\left[\mathbf{1}_{C \cap D} \mid \bigcap_{n}\left(\mathscr{E} \vee \mathscr{D}_{n}\right)\right]$ almost surely (a.s.) and $\mathrm{E}\left[\mathbf{1}_{D} \mid \mathscr{D}_{n}\right]$ converges to $\mathrm{E}\left[\mathbf{1}_{D} \mid \bigcap_{n} \mathscr{D}_{n}\right]$ a.s., we have $\mathrm{E}\left[\mathbf{1}_{C \cap D} \mid \bigcap_{n}\left(\mathscr{E} \vee \mathscr{D}_{n}\right)\right]=\mathbf{1}_{C} \mathrm{E}\left[\mathbf{1}_{D} \mid \bigcap_{n} \mathscr{D}_{n}\right]$ a.s., that is, $\mathrm{E}\left[\mathbf{1}_{C \cap D} \mid \bigcap_{n}(\mathcal{E} \vee\right.$ $\left.\mathscr{D}_{n}\right)$ ] has a version which is $\mathscr{E} \vee \bigcap_{n} \mathscr{D}_{n}$-measurable. By the $\pi-\lambda$ theorem, we can show that, for any $B \in \mathscr{E} \vee \mathscr{D}_{0}, \mathrm{E}\left[\mathbf{1}_{B} \mid \bigcap_{n}\left(\mathscr{E} \vee \mathscr{D}_{n}\right)\right]$ has an $\mathscr{E} \vee \bigcap_{n} \mathscr{D}_{n}$-measurable version. Therefore, $B \in \bigcap_{n}\left(\mathscr{E} \vee \mathscr{D}_{n}\right)$ differs from an $\mathcal{E} \vee \bigcap_{n} \mathscr{D}_{n}$-measurable set by a negligible set. This completes the proof. 
Lemma 3.2. The $\sigma$-field $\bigcap_{k=1}^{\infty} \mathscr{H}_{k}$ is trivial, that is, each of the sets in $\bigcap_{k=1}^{\infty} \mathscr{H}_{k}$ has probability either 0 or 1 .

Proof. The result is essentially Kolmogorov's zero-one law.

Proof of Theorem 2.1. First we prove the theorem for the one-dimensional case. We can see that if (2.3) is true for some $K_{A}>0$ then it is true for any number bigger than $K_{A}$; hence, we can choose $K_{A}>0$ such that (2.3) is true for $K_{A}$ and

$$
A \subset\left\{x \in \mathbb{R}^{d} \mid\|x\|<\frac{K_{A}}{2}\right\} .
$$

Since

$$
\begin{aligned}
l^{-d / 2} & \left(H_{l}(A)-\mathrm{E}\left[H_{l}(A)\right]\right) \\
& =l^{-d / 2}\left(H_{l}(A)-\mathrm{E}\left[H_{l}(A) \mid \mathscr{H}_{\left[l K_{A}\right]}\right]\right)+l^{-d / 2}\left(\mathrm{E}\left[H_{l}(A) \mid \mathscr{H}_{\left[l K_{A}\right]}\right]-\mathrm{E}\left[H_{l}(A)\right]\right) .
\end{aligned}
$$

If we can show that the second term on the right-hand side of the above equality converges to 0 in $L^{2}(\mathrm{P})$ and that the first term converges weakly to the required normal distribution, then we are done.

For each $y \in \mathbb{Z}^{d}$, let $\Delta_{l, y}(A)=\Delta_{l, y}(X, A):=H_{l}\left(\tau_{y}(X), A\right)-H_{l}\left(\tau_{y}\left(X^{o}\right), A\right)$ and $\tilde{\Delta}_{l, y}(A)=H_{l}(X, A)-H_{l}\left(X^{y}, A\right)$. Then $\tilde{\Delta}_{l, y}(A)=\Delta_{l,-y}\left(\tau_{y}(X), A\right)$. By $(2.1)$, there exists a random variable $\Delta_{\infty, y}$ such that, for any sequence $\left\{\left(l_{n}\right) \geq 1\right\}$ and any $B \in \mathcal{R}\left(\mathbb{R}^{d}\right)$,

$$
\tilde{\Delta}_{l_{n}, y}(B)=\Delta_{l_{n}, y}\left(\tau_{y}(X), B\right) \stackrel{\mathrm{P}}{\rightarrow} \Delta_{\infty, y} \quad \text { if } \quad \liminf _{n \rightarrow \infty}\left(\tau_{-y}\left(l_{n} B\right)\right)=\mathbb{R}^{d},
$$

and the definition of $\Delta_{\infty, y}$ in terms of $\tau_{y}(X)$ is the same as that of $\Delta_{\infty}$ in terms of $X$.

Firstly, we show that $l^{-d / 2}\left(\mathrm{E}\left[H_{l}(A) \mid \mathscr{H}_{\left[l K_{A}\right]}\right]-\mathrm{E}\left[H_{l}(A)\right]\right)$ converges to 0 in $L^{2}(\mathrm{P})$ as $l \rightarrow \infty$. Fix $l$. For any $n \geq\left[l K_{A}\right]$, let $\left\{y_{1}^{(n)}, \ldots, y_{m_{n}}^{(n)}\right\}$ be the collection of all the points $y \in \mathbb{Z}^{d}$ such that $\|y\|=n$. Then

$$
\begin{aligned}
\mathrm{E}\left[\left(\mathrm{E}\left[H_{l}(A) \mid \mathcal{H}_{n}\right]-\mathrm{E}\left[H_{l}(A) \mid \mathscr{H}_{n+1}\right]\right)^{2}\right] & \\
= & \sum_{i=1}^{m_{n}} \mathrm{E}\left[\left(\mathrm{E}\left[H_{l}(A) \mid \mathscr{H}_{n+1} \bigvee_{1 \leq j \leq i} \sigma\left\{X_{y_{j}^{(n)}}\right\}\right]\right.\right. \\
& \left.\left.\quad-\mathrm{E}\left[H_{l}(A) \mid \mathscr{H}_{n+1} \bigvee_{1 \leq j \leq i-1} \sigma\left\{X_{y_{j}^{(n)}}\right\}\right]\right)^{2}\right] \\
= & \sum_{i=1}^{m_{n}} \mathrm{E}\left[\left(\mathrm{E}\left[H_{l}(X, A)-H_{l}\left(X^{y_{i}^{(n)}}, A\right) \mid \mathscr{H}_{n+1} \bigvee_{1 \leq j \leq i} \sigma\left\{X_{y_{j}^{(n)}}\right\}\right]\right)^{2}\right] \\
= & \sum_{i=1}^{m_{n}} \mathrm{E}\left[\left(\mathrm{E}\left[\tilde{\Delta}_{l, y_{i}^{(n)}}(A) \mid \mathscr{H}_{n+1} \bigvee_{1 \leq j \leq i} \sigma\left\{X_{y_{j}^{(n)}}\right\}\right]\right)^{2}\right] \\
\leq & \sum_{i=1}^{m_{n}} \mathrm{E}\left[\left(\tilde{\Delta}_{l, y_{i}^{(n)}}(A)\right)^{2}\right] \\
= & \sum_{i=1}^{m_{n}} \mathrm{E}\left[\left(\Delta_{l,-y_{i}^{(n)}}\left(\tau_{y_{i}^{(n)}}(X), A\right)\right)^{2}\right]
\end{aligned}
$$




$$
\begin{aligned}
& =\sum_{i=1}^{m_{n}} \mathrm{E}\left[\left(\Delta_{l,-y_{i}^{(n)}}(A)\right)^{2}\right] \\
& =\sum_{i=1}^{m_{n}} \mathrm{E}\left[\left(\Delta_{l, y_{i}^{(n)}}(A)\right)^{2}\right],
\end{aligned}
$$

where the first equality follows from the martingale property and the last equality is true because the two sets $\left\{y_{1}^{(n)}, \ldots, y_{m_{n}}^{(n)}\right\}$ and $\left\{-y_{1}^{(n)}, \ldots,-y_{m_{n}}^{(n)}\right\}$ are the same. Since

$$
\left\{l^{-d / 2} \mathrm{E}\left[H_{l}(A) \mid \mathscr{H}_{n}\right]: n \geq\left[l K_{A}\right]\right\}
$$

is a backward martingale with respect to $\left\{\mathscr{H}_{n}: n \geq\left[l K_{A}\right]\right\}$ and converges to

$$
l^{-d / 2} \mathrm{E}\left[H_{l}(A) \mid \bigcap_{n} \mathscr{H}_{n}\right] \text { a.s., }
$$

which is equal to $l^{-d / 2} \mathrm{E}\left[H_{l}(A)\right]$ by Lemma 3.2, we have

$$
\begin{aligned}
l^{-d} \mathrm{E}\left[\left(\mathrm{E}\left[H_{l}(A) \mid \mathcal{H}_{\left[l K_{A}\right]}\right]-\mathrm{E}\left[H_{l}(A)\right]\right)^{2}\right] \\
\leq \lim _{n \rightarrow \infty} l^{-d} \mathrm{E}\left[\left(\mathrm{E}\left[H_{l}(A) \mid \mathscr{H}_{\left[l K_{A}\right]}\right]-\mathrm{E}\left[H_{l}(A) \mid \mathscr{H}_{n}\right]\right)^{2}\right] \\
=\lim _{n \rightarrow \infty} l^{-d} \sum_{j=\left[l K_{A}\right]}^{n-1} \mathrm{E}\left[\left(\mathrm{E}\left[H_{l}(A) \mid \mathscr{H}_{j}\right]-\mathrm{E}\left[H_{l}(A) \mid \mathscr{H}_{j+1}\right]\right)^{2}\right] \\
\leq l^{-d} \sum_{n=\left[l K_{A}\right]}^{\infty} \sum_{i=1}^{m_{n}} \mathrm{E}\left[\left(\Delta_{l, y_{i}^{(n)}}^{H}(A)\right)^{2}\right] \\
=\frac{1}{l^{d}} \sum_{\substack{y \in \mathbb{Z}^{d} \\
\|y\| \geq l K_{A}}} \mathrm{E}\left[\left(\Delta_{l, y}^{H}(A)\right)^{2}\right],
\end{aligned}
$$

which goes to zero by (2.3). The first inequality follows from Fatou's Lemma. Therefore, $l^{-d / 2}\left(\mathrm{E}\left[H_{l}(A) \mid \mathscr{H}_{\left[l K_{A}\right]}\right]-\mathrm{E}\left[H_{l}(A)\right]\right) \rightarrow 0$ in $L^{2}(\mathrm{P})$.

Now we use Penrose's idea to prove that $l^{-d / 2}\left(H_{l}(A)-\mathrm{E}\left[H_{l}(A) \mid \mathscr{H}_{\left[l K_{A}\right]}\right]\right)$ converges to a normal distribution. Let $\left\{z_{1}, \ldots, z_{n_{l}}\right\}$ be the set of all the points $z \in \mathbb{Z}^{d}$ such that $\|z\|<\left[l K_{A}\right]$ is ordered by lexicographic ordering on $\mathbb{Z}^{d}$ from the smallest to the largest. Then

$$
\begin{aligned}
H_{l}(A) & -\mathrm{E}\left[H_{l}(A) \mid \mathscr{H}_{\left[l K_{A}\right]}\right] \\
= & \sum_{i=1}^{n_{l}}\left(\mathrm{E}\left[H_{l}(A) \mid \mathscr{H}_{\left[l K_{A}\right]} \bigvee_{1 \leq j \leq i} \sigma\left\{X_{z_{j}}\right\}\right]-\mathrm{E}\left[H_{l}(A) \mid \mathscr{H}_{\left[l K_{A}\right]} \bigvee_{1 \leq j \leq i-1} \sigma\left\{X_{z_{j}}\right\}\right]\right) \\
= & \sum_{i=1}^{n_{l}} \mathrm{E}\left[\tilde{\Delta}_{l, z_{i}}(A) \mid \mathscr{H}_{\left[l K_{A}\right]} \bigvee_{1 \leq j \leq i} \sigma\left\{X_{z_{j}}\right\}\right] \\
= & \sum_{i=1}^{n_{l}} \mathrm{E}\left[\tilde{\Delta}_{l, z_{i}}(A) \mid \mathscr{H}_{\left[l K_{A}\right]} \vee \mathcal{F}_{z_{i}}\right] .
\end{aligned}
$$


Let $D_{z_{i}}^{l}=\mathrm{E}\left[\tilde{\Delta}_{l, z_{i}}(A) \mid \mathcal{H}_{\left[l K_{A}\right]} \vee \mathcal{F}_{z_{i}}\right]$, and let $D_{z}=\mathrm{E}\left[\Delta_{\infty, z} \mid \mathcal{F}_{z}\right]$ for each $z \in \mathbb{Z}^{d}$. Then $\left\{D_{z_{i}}^{l} \mid i=1, \ldots, n_{l}\right\}$ are martingale differences and the definition of $D_{z}$ in terms of $\tau_{z}(X)$ is the same as that of $D_{o}$ in terms of $X$.

Now we show that

$$
l^{-d} \sum_{i=1}^{n_{l}}\left(D_{z_{i}}^{l}\right)^{2} \stackrel{L^{1}}{\longrightarrow} \mathrm{E}\left[\mathrm{E}\left[\left(\Delta_{\infty} \mid \mathcal{F}_{o}\right)^{2}\right]\right]|A|
$$

Set

$$
\sup \left\{\mathrm{E}\left[\left|\Delta_{l,-y}^{H}(B)\right|^{\gamma}\right]: B \in \mathcal{R}\left(\mathbb{R}^{d}\right), l \geq 1, y \in \mathbb{Z}^{d}\right\}=M_{1}<\infty,
$$

and note that

$$
l^{-d} \sum_{i=1}^{n_{l}}\left(D_{z_{i}}^{l}\right)^{2}=l^{-d}\left(\sum_{\substack{z_{i} \in(l A) \\\left|z_{i}-\partial(l A)\right|>\sqrt{l}}}\left(D_{z_{i}}^{l}\right)^{2}+\sum_{\substack{z_{i} \notin(l A) \\\left|z_{i}-\partial(l A)\right|>\sqrt{l}}}\left(D_{z_{i}}^{l}\right)^{2}+\sum_{\left|z_{i}-\partial(l A)\right| \leq \sqrt{l}}\left(D_{z_{i}}^{l}\right)^{2}\right) .
$$

First, we show that

$$
\lim _{l \rightarrow \infty} \sup _{\substack{z_{i} \in(l A) \\\left|z_{i}-\partial(l A)\right|>\sqrt{l}}}\left|\mathrm{E}\left[\left(D_{z_{i}}^{l}\right)^{2}\right]-\mathrm{E}\left[\left(D_{z_{i}}\right)^{2}\right]\right|=0 \quad \text { and } \lim _{l \rightarrow \infty} \sup _{\substack{z_{i} \notin(l A) \\\left|z_{i}-\partial(l A)\right|>\sqrt{l}}}\left|\mathrm{E}\left[\left(D_{z_{i}}^{l}\right)^{2}\right]\right|=0 .
$$

Let $\left\{\left(l_{n}, z_{i_{n}}^{n}\right) \mid l_{n} \geq 1, z_{i_{n}}^{n} \in \mathbb{Z}^{d}, n \geq 1\right\}$ be a sequence such that $\lim _{n \rightarrow \infty} l_{n}=\infty, z_{i_{n}}^{n} \in$ $l_{n} A$, and $\left|z_{i_{n}}^{n}-\partial\left(l_{n} A\right)\right|>\sqrt{l_{n}}$. Because $\left\{y \in \mathbb{R}^{d}:\left|y-z_{i_{n}}^{n}\right| \leq \sqrt{l_{n}}\right\} \subset l_{n} A$, we have $\{y \in$ $\left.\mathbb{R}^{d}:|y| \leq \sqrt{l_{n}}\right\} \subset l_{n} A-z_{i_{n}}^{n}$ and, hence, $\liminf _{n \rightarrow \infty}\left(l_{n} A-z_{i_{n}}^{n}\right)=\mathbb{R}^{d}$. By (2.1) and (2.4), $\mathrm{E}\left[\left(\Delta_{l_{n},-z_{i_{n}}^{n}}(A)-\Delta_{\infty}\right)^{2}\right] \rightarrow 0$. Now we compute

$$
\begin{aligned}
& \mathrm{E}\left[\left(D_{z_{i_{n}}^{n}}^{l_{n}}-D_{z_{i_{n}}^{n}}\right)^{2}\right] \\
& =\mathrm{E}\left[\left(\mathrm{E}\left[\tilde{\Delta}_{l_{n}, z_{i_{n}}^{n}}(A) \mid \mathscr{H}_{\left[l_{n} K_{A}\right]} \vee \mathcal{F}_{z_{i_{n}}^{n}}\right]-\mathrm{E}\left[\Delta_{\infty, z_{i_{n}}^{n}} \mid \mathcal{F}_{z_{i_{n}}^{n}}\right]\right)^{2}\right] \\
& \leq \mathrm{E}\left[\left(\mathrm{E}\left[\tilde{\Delta}_{l_{n}, z_{i_{n}}^{n}}(A) \mid \mathscr{H}_{\left[l_{n} K_{A}\right]} \vee \mathcal{F}_{z_{i_{n}}^{n}}\right]-\mathrm{E}\left[\Delta_{\infty, z_{i n}^{n}} \mid \mathscr{H}_{\left[l_{n} K_{A}\right]} \vee \mathcal{F}_{z_{i_{n}}^{n}}\right]\right.\right. \\
& \left.\left.+\mathrm{E}\left[\Delta_{\infty, z_{i n}^{n}} \mid \mathcal{H}_{\left[l_{n} K_{A}\right]} \vee \mathcal{F}_{z_{i n}^{n}}\right]-\mathrm{E}\left[\Delta_{\infty, z_{i n}^{n}} \mid \mathcal{F}_{z_{i n}^{n}}\right]\right)^{2}\right] \\
& \leq 2 \mathrm{E}\left[\left(\mathrm{E}\left[\tilde{\Delta}_{l_{n}, z_{i_{n}}^{n}}(A)-\Delta_{\infty, z_{i_{n}}^{n}} \mid \mathcal{H}_{\left[l_{n} K_{A}\right]} \vee \mathcal{F}_{z_{i_{n}}^{n}}\right]\right)^{2}\right] \\
& +2 \mathrm{E}\left[\left(\mathrm{E}\left[\Delta_{\infty, z_{i n}^{n}} \mid \mathscr{H}_{\left[l_{n} K_{A}\right]} \vee \mathcal{F}_{z_{i n}^{n}}\right]-\mathrm{E}\left[\Delta_{\infty, z_{i n}^{n}} \mid \mathcal{F}_{z_{i n}^{n}}\right]\right)^{2}\right] \\
& \leq 2 \mathrm{E}\left[\left(\tilde{\Delta}_{l_{n}, z_{i_{n}}^{n}}(A)-\Delta_{\infty, z_{i_{n}}^{n}}\right)^{2}\right] \\
& +2 \mathrm{E}\left[\left(\mathrm{E}\left[\Delta_{\infty, z_{i n}^{n}} \mid \mathscr{H}_{\left[l_{n} K_{A}\right]} \vee \mathcal{F}_{z_{i_{n}}^{n}}\right]-\mathrm{E}\left[\Delta_{\infty, z_{i_{n}}^{n}} \mid \mathcal{F}_{z_{i_{n}}^{n}}\right]\right)^{2}\right] \\
& =2 \mathrm{E}\left[\left(\Delta_{l_{n},-z_{i_{n}}^{n}}\left(\tau_{z_{i_{n}}^{n}}(X), A\right)-\Delta_{\infty}\left(\tau_{z_{i_{n}}^{n}}(X)\right)\right)^{2}\right] \\
& +2 \mathrm{E}\left[\left(\mathrm{E}\left[\Delta_{\infty, z_{i n}^{n}} \mid \mathscr{H}_{\left[l_{n} K_{A}\right]} \vee \mathcal{F}_{z_{i_{n}}^{n}}\right]-\mathrm{E}\left[\Delta_{\infty, z_{i n}^{n}} \mid \mathcal{F}_{z_{i_{n}}^{n}}\right]\right)^{2}\right] \\
& =2 \mathrm{E}\left[\left(\Delta_{l_{n},-z_{i n}^{n}}(A)-\Delta_{\infty}\right)^{2}\right]+2 \mathrm{E}\left[\left(\mathrm{E}\left[\Delta_{\infty} \mid g_{n} \vee \mathcal{F}_{o}\right]-\mathrm{E}\left[\Delta_{\infty} \mid \mathcal{F}_{o}\right]\right)^{2}\right],
\end{aligned}
$$

where $g_{n}=\sigma\left\{X_{y} \mid 0 \prec y,\left\|y+z_{i_{n}}^{n}\right\| \geq\left[l_{n} K_{A}\right]\right\}$ is independent of $\mathcal{F}_{o}$. Since $z_{i_{n}}^{n} \in l_{n} A$, by (3.1) we have $\left\|z_{i_{n}}^{n}\right\|<\left[l_{n} K_{A}\right] / 2$. Hence, if $\left\|y+z_{i_{n}}^{n}\right\| \geq\left[l_{n} K_{A}\right]$ then $\|y\| \geq\left[l_{n} K_{A}\right] / 2 \geq\left[l_{n} K_{A} / 2\right]$, and we have $g_{n} \subset \mathscr{H}_{\left[l_{n} K_{A} / 2\right]} \cap \sigma\left\{X_{y} \mid 0 \prec y\right\}$. On the other hand, if $\|y\| \geq 2\left[l_{n} K_{A}\right]$ then 
$\left\|y+z_{i_{n}}^{n}\right\| \geq\left[l_{n} K_{A}\right]$, and we have $\mathscr{H}_{2\left[l_{n} K_{A}\right]} \cap \sigma\left\{X_{y} \mid 0 \prec y\right\} \subset g_{n}$. Because $l_{n} \rightarrow \infty$, we can choose a subsequence of $g_{n}$, which is decreasing. Without loss of generality, we assume that $g_{n}$ is itself a decreasing sequence. By Lemma $3.2, \bigcap_{n} g_{n} \subset \bigcap_{n} \mathscr{H}_{\left[l_{n} K_{A} / 2\right]}$ is trivial; hence, $\bigcap_{n}\left(g_{n} \vee \mathcal{F}_{o}\right)=\left(\bigcap_{n} g_{n}\right) \vee \widetilde{F}_{o}=\mathscr{F}_{o}$ up to negligible sets, by Lemma 3.1. Therefore, we have $\mathrm{E}\left[\left(\mathrm{E}\left[\Delta_{\infty} \mid g_{n} \vee \mathcal{F}_{o}\right]-\mathrm{E}\left[\Delta_{\infty} \mid \mathcal{F}_{o}\right]\right)^{2}\right] \rightarrow 0$, by the backward martingale theorem. Now we have $\mathrm{E}\left[\left(D_{z_{i_{n}}^{n}}^{l_{n}}-D_{z_{i_{n}}^{n}}\right)^{2}\right] \rightarrow 0$ and

$$
\begin{aligned}
\left|\mathrm{E}\left[\left(D_{z_{i n}}^{l_{n}}\right)^{2}\right]-\mathrm{E}\left[\left(D_{z_{i_{n}}^{n}}\right)^{2}\right]\right| & =\left|\mathrm{E}\left[\left(D_{z_{i_{n}}}^{l_{n}}-D_{z_{i_{n}}^{n}}\right)\left(D_{z_{i_{n}}}^{l_{n}}+D_{z_{i_{n}}^{n}}\right)\right]\right| \\
& \leq\left(\mathrm{E}\left[\left(D_{z_{i_{n}}}^{l_{n}}-D_{z_{i_{n}}^{n}}\right)^{2}\right] \mathrm{E}\left[\left(D_{z_{i_{n}}}^{l_{n}}+D_{z_{i_{n}}^{n}}\right)^{2}\right]\right)^{1 / 2} \\
& \leq 2 M_{1}^{1 / \gamma}\left(\mathrm{E}\left[\left(D_{z_{i_{n}}}^{l_{n}}-D_{z_{i_{n}}^{n}}\right)^{2}\right]\right)^{1 / 2} \\
& \rightarrow 0,
\end{aligned}
$$

where the last inequality follows from Condition 2.2 and Jensen's inequality. Hence,

$$
\lim _{l \rightarrow \infty} \sup _{\substack{z_{i} \in l A \\\left|z_{i}-\partial(l A)\right|>\sqrt{l}}}\left|\mathrm{E}\left[\left(D_{z_{i}}^{l}\right)^{2}\right]-\mathrm{E}\left[\left(D_{z_{i}}\right)^{2}\right]\right|=0
$$

and, similarly, we can show that

$$
\lim _{l \rightarrow \infty} \sup _{\substack{z_{i} \notin l A \\\left|z_{i}-\partial(l A)\right|>\sqrt{l}}}\left|\mathrm{E}\left[\left(D_{z_{i}}^{l}\right)^{2}\right]\right|=0 .
$$

Now we have

$$
\begin{gathered}
l^{-d} \sum_{\substack{z_{i} \in l A \\
\left|z_{i}-\partial(l A)\right|>\sqrt{l}}}\left|\mathrm{E}\left[\left(D_{z_{i}}^{l}\right)^{2}\right]-\mathrm{E}\left[\left(D_{z_{i}}\right)^{2}\right]\right| \rightarrow 0, \\
l^{-d} \sum_{\substack{z_{i} \notin l A \\
\left|z_{i}-\partial(l A)\right|>\sqrt{l}}} \mathrm{E}\left[\left(D_{z_{i}}^{l}\right)^{2}\right] \rightarrow 0, \\
l^{-d} \sum_{\left|z_{i}-\partial(l A)\right| \leq \sqrt{l}} \mathrm{E}\left[\left(D_{z_{i}}^{l}\right)^{2}\right] \rightarrow 0 .
\end{gathered}
$$

Equation (3.4) holds by the moment condition and the fact that the Lebesgue measure of $\partial A$ is 0 . By the ergodic theorem,

$$
l^{-d} \sum_{\substack{z_{i} \in l A \\\left|z_{i}-\partial(l A)\right|>\sqrt{l}}}\left(D_{z_{i}}\right)^{2} \stackrel{L^{1}}{\longrightarrow} \mathrm{E}\left[\mathrm{E}\left[\left(\Delta_{\infty} \mid \mathcal{F}_{0}\right)^{2}\right]\right]|A|,
$$

and by (3.2)-(3.4) we have

$$
l^{-d} \sum_{i=1}^{n_{l}}\left(D_{z_{i}}^{l}\right)^{2} \stackrel{L^{1}}{\longrightarrow} \mathrm{E}\left[\mathrm{E}\left[\left(\Delta_{\infty} \mid \mathcal{F}_{0}\right)^{2}\right]\right]|A| .
$$

Because

$$
\mathrm{E}\left[\left(\max _{1 \leq i \leq n_{l}}\left(l^{-d / 2}\left|D_{z_{i}}^{l}\right|\right)\right)^{\gamma}\right] \leq l^{-\gamma d / 2} \mathrm{E}\left[\sum_{i=1}^{n_{l}}\left|D_{z_{i}}^{l}\right|^{\gamma}\right] \leq \frac{\left(2\left[l K_{A}\right]+1\right)^{d} M_{1}}{l^{\gamma d / 2}} \rightarrow 0 \quad \text { as } l \rightarrow \infty
$$


we have

$$
\begin{gathered}
\sup _{l \geq 1} \mathrm{E}\left[\max _{1 \leq i \leq n_{l}}\left(l^{-d / 2}\left|D_{z_{i}}^{l}\right|\right)^{2}\right] \leq \sup _{l \geq 1}\left[\mathrm{E}\left[\left(\max _{1 \leq i \leq n_{l}}\left(l^{-d / 2}\left|D_{z_{i}}^{l}\right|\right)\right)^{\gamma}\right]\right]^{2 / \gamma}<\infty, \\
l^{-d / 2} \max _{1 \leq i \leq n_{l}}\left|D_{z_{i}}^{l}\right| \rightarrow 0 \quad \text { in probability. }
\end{gathered}
$$

Hence, by (3.5)-(3.7) and Theorem (2.3) of McLeish (1974), we have the required CLT. For the multidimensional case, we can prove the CLT for any linear combination by the same martingale argument as the one-dimensional case. Then by the Cramer-Wold device we can obtain the multidimensional CLT.

\section{Proof of Theorem 2.2}

We will prove our functional CLT by the following steps. Firstly, we will prove the CLT for $\eta_{t}(l A)$ as $l \rightarrow \infty$, where $A$ is any Riemann measurable subset, and $t \geq 0$. In order to do this, we apply Theorem 2.1 to the stochastic equations of Garcia and Kurtz (2006). Note that $\eta_{t}(l A)$ can be written as $\int_{\mathbb{R}^{d}} \mathbf{1}_{A}(x / l) \eta_{t}(\mathrm{~d} x)$. Hence, by using the linear combinations of indicator functions of Riemann measurable subsets to approximate a general bounded and integrable function $f$, we can prove the weak convergence of finite-dimensional distributions of $Y^{(l)}(f, t)$. Secondly, we will prove the relative compactness of

$$
\left\{\left(Y^{(l)}\left(f_{1}, t\right), \ldots, Y^{(l)}\left(f_{n}, t\right)\right): l \geq 1\right\} .
$$

In Garcia and Kurtz (2006) spatial birth and death processes were obtained as solutions of a system of stochastic equations. The processes were required to be locally finite, that is, the number of points in each compact subset was finite. But there could be infinitely many points over the full (noncompact) space. The state space and its topology for the processes are given as follows. We will use them in this paper.

Let $F_{1} \subset F_{2} \subset \cdots$ satisfy $\bigcup_{k} F_{k}=\mathbb{R}^{d}$, and let $c_{k}$ be bounded continuous functions on $\mathbb{R}^{d}$ which satisfy $c_{k} \geq 0$ and $\inf _{x \in F_{k}} c_{k}(x)>0$. Define the state space

$$
\S=\left\{\xi \in \mathcal{N}\left(\mathbb{R}^{d}\right): \int_{\mathbb{R}^{d}} c_{k}(x) \xi(\mathrm{d} x)<\infty, k=1,2, \ldots\right\} .
$$

Assume that $c_{1} \leq c_{2} \leq \cdots$. Let

$$
\mathcal{C}=\left\{f \in \bar{C}\left(\mathbb{R}^{d}\right):|f| \leq a c_{k} \text { for some } k \text { and } a>0\right\},
$$

and topologize $\delta$ by the weak topology generated by $\mathcal{C}$. Then $\delta$ is a separable metric space. Here $D_{\S}[0, \infty)$ will denote the space of càdlàg $\&$-valued functions, i.e. those which are right continuous and have left limits, with the Skorokhod $\left(J_{1}\right)$ topology.

In this paper we assume that the death rate is equal to a nonnegative constant $b_{0}$. If $b_{0}=0$, the process is the spatial pure-birth process. Let $N$ denote a Poisson random measure on $\mathbb{R}^{d} \times[0, \infty)^{3}$ with mean measure $\mathrm{d} x \times \mathrm{d} s \times \mathrm{e}^{-r} \mathrm{~d} r \times \mathrm{d} u$ independent of $\eta_{0}$. Here $\eta_{0}$ is the initial point process in Theorem 2.2. Let $\hat{\eta}_{0}$ be the point process on $\mathbb{R}^{d} \times[0, \infty)$ obtained by associating to each 'count' in $\eta_{0}$ an independent, unit exponential random variable. Then the spatial birth and death process $\eta_{t}$ satisfies a stochastic equation of the form

$$
\begin{aligned}
\eta_{t}(A)= & \int_{A \times[0, t] \times[0,+\infty)^{2}} \mathbf{1}_{\left[0, \lambda\left(x, \eta_{s-}\right)\right]}(u) \mathbf{1}_{(t-s, \infty)}(r) N(\mathrm{~d} x, \mathrm{~d} s, \mathrm{~d} r, \mathrm{~d} u) \\
& +\int_{A \times[0, \infty)} \mathbf{1}_{\left(b_{0} t, \infty\right)}(r) \hat{\eta}_{0}(\mathrm{~d} x, \mathrm{~d} r) .
\end{aligned}
$$


The following two conditions given in Garcia and Kurtz (2006) guarantee that the integral on the right-hand side of (4.1) exists and determines an $\&$-valued process with sample paths in $D_{s}[0, \infty)$.

Condition 4.1. (Garcia and Kurtz (2006).) For each compact $\mathcal{K} \subset$,

$$
\sup _{\zeta \in \mathcal{K}} \int_{\mathbb{R}^{d}} c_{k}(x) \lambda(x, \zeta) \mathrm{d} x<\infty, \quad k=1,2, \ldots
$$

Condition 4.2. (Garcia and Kurtz (2006).) If $\lim _{n \rightarrow \infty} \int_{\mathbb{R}^{d}} c_{k}(x)\left|\zeta_{n}-\zeta\right|(\mathrm{d} x)=0$ for each $k=1,2, \ldots$ then $\lambda(x, \zeta)=\lim _{n \rightarrow \infty} \lambda\left(x, \zeta_{n}\right)$.

We say that $N$ is compatible with respect to a filtration $\left\{\mathscr{F}_{t}\right\}$ if, for any measurable subset $A$ in $\mathbb{R}^{d} \times[0, \infty)^{2}, N(A,[0, t])$ is $\mathcal{F}_{t}$-measurable and $N(A,(t, s])$ is independent of $\mathcal{F}_{t}$, where $0 \leq t<s$. A solution of (4.1) is a process satisfying the following properties.

(i) It is a process with sample paths in $D_{\S}[0, \infty)$.

(ii) It is adapted to a filtration $\left\{\mathcal{F}_{t}\right\}$ with respect to which $N$ is compatible such that (4.1) holds a.s. for all Borel subsets $A$ and $t \geq 0$ (allowing $\infty=\infty$ ).

The following theorem is a combination of Theorem 2.13 and Lemma 3.14 of Garcia and Kurtz (2006).

Theorem 4.1. (Garcia and Kurtz (2006).) Assume that $\lambda$ is translation invariant and satisfies Conditions 4.1 and 4.2, and that $\eta_{0}$ is translation invariant. Suppose that there exists a measurable function $a(x, y)$ on $\mathbb{R}^{d} \times \mathbb{R}^{d}$ satisfying

$$
a(x, y) \geq \sup _{\eta}\left|\lambda\left(x, \eta+\delta_{y}\right)-\lambda(x, \eta)\right|
$$

and that there exists a positive and bounded function $h(x)$ such that

$$
M^{\prime}=\sup _{x \in \mathbb{R}^{d}} \int_{\mathbb{R}^{d}} \frac{h(x) a(x, y)}{h(y)} \mathrm{d} y<\infty .
$$

Then there exists a unique solution to (4.1) and $\eta_{t}$ is translation invariant.

Remarks 4.1. (a) The boundedness of $h(x)$ was not included in Garcia and Kurtz (2006). However, in the proof of the above theorem in Garcia and Kurtz (2006) the finiteness of the supremum of the product of $h(x)$ and some integral was used implicitly, which cannot be derived without the boundedness of $h(x)$.

(b) The following inequality is Lemma 2.15 of Garcia and Kurtz (2006): for any $\eta^{1}, \eta^{2} \in \delta$,

$$
\left|\lambda\left(x, \eta^{1}\right)-\lambda\left(x, \eta^{2}\right)\right| \leq \int_{\mathbb{R}^{d}} a(x, y)\left|\eta^{1}-\eta^{2}\right|(\mathrm{d} y),
$$

where $\left|\eta^{1}-\eta^{2}\right|(\mathrm{d} y)$ denotes the total variation of $\eta^{1}-\eta^{2}$. We will use this inequality in the following. 
(c) In Theorem 2.2 we assume that $\lambda(x, \zeta)$ is bounded. We set $c_{k}(x)$ to be integrable. Then Condition 4.1 is satisfied. Moreover, we set $c_{1}(x)$ to be $1 /\left(1+|x|^{2 d+\delta}\right)$. For each $x$, we can find a number $k(x)$ which depends only on $x$, such that

$$
a(x, y) \leq k(x) c_{1}(y) \text { for all } y .
$$

Then Condition 4.2 is satisfied. In fact, if $\lim _{n \rightarrow \infty} \int_{S} c_{1}(x)\left|\zeta_{n}-\zeta\right|(\mathrm{d} x)=0$,

$$
\left|\lambda(x, \zeta)-\lambda\left(x, \zeta_{n}\right)\right| \leq \int_{S} a(x, y)\left|\zeta_{n}-\zeta\right|(\mathrm{d} y) \leq k(x) \int_{S} c_{1}(y)\left|\zeta_{n}-\zeta\right|(\mathrm{d} y) \rightarrow 0 .
$$

(d) From the proof in Garcia and Kurtz (2006), we can see that the unique solution is adapted to the filtration generated by $N$ and $\hat{\eta}$.

Here we only prove the theorem for a unit death rate because, for other positive constants, the proof is the same and, for the spatial pure-birth process, the proof is similar and simpler. Under the conditions of Theorem $2.2, \lambda$ is bounded by $L$ and $\sup _{x} \int_{\mathbb{R}^{d}} a(x, y) \mathrm{d} y<\infty$. Hence, we can set $h(x)=1$. Then we have $\sup _{x} \int_{\mathbb{R}^{d}}(h(x) a(x, y) / h(y)) \mathrm{d} y<\infty$ and, by Theorem 4.1 and Remarks 4.1, the stochastic equation (4.1) has a unique solution which has the same distribution as our spatial birth and death process $\eta_{t}$. We can just assume that $\eta_{t}$ is the solution of (4.1). By Remark 4.1(d), $\eta_{t}$ is adapted to the filtration generated by $N$ and $\hat{\eta}_{0}$. For any set $E \subset \mathbb{R}^{d} \times[0, \infty)^{3}$ (or $\mathbb{R}^{d} \times[0, \infty)$ ), we use $\left.N\right|_{E}\left(\right.$ or $\left.\left.\hat{\eta}_{0}\right|_{E}\right)$ to denote the restriction of $N$ (or $\hat{\eta}_{0}$ ) to $E$. Let $B_{0}=\prod_{i=1}^{d}\left[-\frac{1}{2}, \frac{1}{2}\right.$ ) be the unit cube with its center at the origin in $\mathbb{R}^{d}$. Then $B_{0}+z$ is the unit cube with its center at $z \in \mathbb{Z}^{d}$. We define a family of independent, identically distributed random elements $X=\left(X_{z}, z \in \mathbb{Z}^{d}\right)$, where

$$
X_{z}=\left(\tau_{-z}\left(\left.N\right|_{\left(B_{0}+z\right) \times[0, \infty)^{3}}\right), \tau_{-z}\left(\left.\hat{\eta}_{0}\right|_{\left(B_{0}+z\right) \times[0, \infty)}\right)\right)
$$

and $\tau_{-z}$ denotes the shift operator. Let $N_{0}$ be a Poisson random measure on $B_{0} \times[0, \infty)^{3}$ independent of $N$ which has the same distribution as $\left.N\right|_{B_{0} \times[0, \infty)^{3}}$, and let $N^{0}$ be the Poisson random measure obtained from $N$ by replacing $\left.N\right|_{B_{0} \times[0, \infty)^{3}}$ with $N_{0}$. Let $\hat{\eta}_{0,0}$ be a random measure independent of $\hat{\eta}_{0}$, having the same distribution as $\left.\hat{\eta}_{0}\right|_{B_{0} \times[0, \infty)}$, and let $\hat{\eta}_{0}^{0}$ be $\hat{\eta}_{0}$ with the restriction to $B_{0} \times[0, \infty)$ replaced by $\hat{\eta}_{0,0}$. Let $X_{*}=\left(N_{0}, \hat{\eta}_{0,0}\right)$. Then $X_{*}$ has the same distribution as $X_{0}$ and is independent of $\left(X_{z}\right)$. Let $X^{o}$ be the family $X$ with $X_{0}$ replaced by $X_{*}$ and all other entries the same as $X$.

Since the function $c(x)$ in the conditions of Theorem 2.2 is bounded in a neighborhood of the origin in $\mathbb{R}^{d}$, without loss of generality, we assume that $c(x)$ is bounded in $B_{0}$. Otherwise, we can replace the lattice $\mathbb{Z}^{d}$ by $\varepsilon \mathbb{Z}^{d}$ for a sufficiently small $\varepsilon>0$.

Let

$$
\xi_{t}^{(l)}(A)=\frac{1}{l^{d / 2}}\left(\eta_{t}(l A)-\mathrm{E}\left[\eta_{t}(l A)\right]\right),
$$

where $1 \leq l<\infty$. For any $0 \leq t_{1} \leq \cdots \leq t_{n}$ and Riemann measurable sets $A_{1}, \ldots, A_{n}$, we will use Theorem 2.1 to show that $\left(\xi_{t_{1}}^{(l)}\left(A_{1}\right), \ldots, \xi_{t_{n}}^{(l)}\left(A_{n}\right)\right)$ converges weakly to some multivariate normal distribution. Let $H_{l}(X, A)=\eta_{t}(l A)$, where $t \geq 0$ and $A$ is a Riemann measurable set in $\mathbb{R}^{d}$, and, for any $z \in \mathbb{Z}^{d}$, let $H_{l, z}(X, A)=H_{l}\left(\tau_{z}(X), A\right)=\eta_{t}(l A+z)$. Therefore,

$$
\Delta_{l, z}^{H}(A)=H_{l, z}(X, A)-H_{l, z}\left(X^{o}, A\right)=\eta_{t}(l A+z)-\eta_{t}^{0}(l A+z),
$$

where $\eta_{t}^{0}$ is the unique solution of (4.1) with $N$ replaced by $N^{0}$. We have to check that Theorem 2.1 satisfies Conditions 2.1 and 2.2. 
Lemma 4.1. Suppose that $\lambda$ is bounded by L and that $\sup _{x} \int_{\mathbb{R}^{d}} a(x, y) \mathrm{d} y<\infty$. Then, for any $t \geq 0$, we have

$$
\sup _{x \in \mathbb{R}^{d}} c(x) \mathrm{E}\left[\int_{\mathbb{R}^{d}} a(x, y)\left|\eta_{t}-\eta_{t}^{0}\right|(\mathrm{d} y)\right] \leq 2\left(L+\mu_{1}\right)\left[\sup _{z \in B_{0}} c(z)\right] \mathrm{e}^{t M}
$$

Proof. For any $x \in \mathbb{R}^{n}$, we have

$$
\begin{aligned}
& \mathrm{E}\left[\int a(x, y)\left|\eta_{t}-\eta_{t}^{0}\right|(\mathrm{d} y)\right] \\
& \leq \mathrm{E}\left[\int_{0}^{t} \int_{B_{0}^{\mathrm{c}} \times[0,+\infty)^{2}} a(x, y)\left|\mathbf{1}_{\left[0, \lambda\left(y, \eta_{s-}\right)\right]}(u)-\mathbf{1}_{\left[0, \lambda\left(y, \eta_{s-}^{0}\right)\right]}(u)\right|\right. \\
&\left.\times \mathbf{1}_{(t-s, \infty)}(r) N(\mathrm{~d} y, \mathrm{~d} s, \mathrm{~d} r, \mathrm{~d} u)\right] \\
& \quad+\mathrm{E}\left[\int_{0}^{t} \int_{B_{0} \times[0,+\infty)^{2}} a(x, y) \mathbf{1}_{\left[0, \lambda\left(y, \eta_{s-}\right)\right]}(u) \mathbf{1}_{(t-s, \infty)}(r) N(\mathrm{~d} y, \mathrm{~d} s, \mathrm{~d} r, \mathrm{~d} u)\right] \\
& \quad+\mathrm{E}\left[\int_{0}^{t} \int_{B_{0} \times[0,+\infty)^{2}} a(x, y) \mathbf{1}_{\left[0, \lambda\left(y, \eta_{s-}^{0}\right)\right]}(u) \mathbf{1}_{(t-s, \infty)}(r) N^{0}(\mathrm{~d} y, \mathrm{~d} s, \mathrm{~d} r, \mathrm{~d} u)\right] \\
& \quad+\mathrm{E}\left[\int_{B_{0} \times[0, \infty)} a(x, y) \mathbf{1}_{(t, \infty)}(r) \hat{\eta}_{0}(\mathrm{~d} y, \mathrm{~d} r)\right] \\
& \quad+\mathrm{E}\left[\int_{B_{0} \times[0, \infty)} a(x, y) \mathbf{1}_{(t, \infty)}(r) \hat{\eta}_{0}^{0}(\mathrm{~d} y, \mathrm{~d} r)\right] \\
& \leq \int_{0}^{t} \int_{\mathbb{R}^{d}} a(x, y) \mathrm{E}\left[\left|\lambda\left(y, \eta_{s}\right)-\lambda\left(y, \eta_{s}^{0}\right)\right|\right] \mathrm{e}^{-(t-s)} \mathrm{d} y \mathrm{~d} s \\
&+2 L \int_{0}^{t} \int_{B_{0}} a(x, y) \mathrm{e}^{-(t-s)} \mathrm{d} y \mathrm{~d} s \\
&+\mathrm{E}\left[\int_{B_{0}} a(x, y) \eta_{0}(\mathrm{~d} y)\right]+\mathrm{E}\left[\int_{B_{0}} a(x, y) \eta_{0}^{0}(\mathrm{~d} y)\right] \\
& \leq \int_{0}^{t} a(x, y) \mathrm{E}\left[\int a(y, z)\left|\eta_{s}-\eta_{s}^{0}\right|(\mathrm{d} z)\right] \mathrm{e}^{-(t-s)} \mathrm{d} y \mathrm{~d} s+2\left(L+\mu_{1}\right) \int_{B_{0}} a(x, y) \mathrm{d} y .
\end{aligned}
$$

By iteration, for any positive integer $n$, we have

$$
\begin{aligned}
& \mathrm{E}\left[\int a(x, y)\left|\eta_{t}-\eta_{t}^{0}\right|(\mathrm{d} y)\right] \\
& \leq 2\left(L+\mu_{1}\right)\left(\int a(x, y) \mathrm{d} y+\int_{0}^{t} \mathrm{e}^{-(t-s)} \mathrm{d} s \iint_{B_{0}} a(x, y) a\left(y, y_{1}\right) \mathrm{d} y_{1} \mathrm{~d} y+\cdots\right. \\
& \left.\quad+\int_{0}^{t} \frac{(t-s)^{n}}{n !} \mathrm{e}^{-(t-s)} \mathrm{d} s \int \cdots \int_{B_{0}} a(x, y) a\left(y, y_{1}\right) \cdots a\left(y_{n-1}, y_{n}\right) \mathrm{d} y_{n} \cdots \mathrm{d} y_{1} \mathrm{~d} y\right) \\
& \quad+R_{n},
\end{aligned}
$$


where

$$
\begin{aligned}
R_{n}= & \int \cdots \int_{B_{0}} a(x, y) a\left(y, y_{1}\right) \cdots a\left(y_{n-1}, y_{n}\right) \\
& \times \int_{0}^{t} \mathrm{E}\left[\int a\left(y_{n}, y_{n+1}\right)\left|\eta_{t}-\eta_{t}^{0}\right|\left(\mathrm{d} y_{n+1}\right)\right] \frac{(t-s)^{n}}{n !} \mathrm{e}^{-(t-s)} \mathrm{d} s \mathrm{~d} y_{n+1} \mathrm{~d} y_{n} \cdots \mathrm{d} y_{1} \mathrm{~d} y
\end{aligned}
$$

is the remainder term. We estimate $R_{n}$. Let $K=\sup _{x} \int_{\mathbb{R}^{d}} a(x, y) \mathrm{d} y<\infty$. Because

$$
\begin{aligned}
& \mathrm{E}\left[\int a\left(y_{n}, y_{n+1}\right)\left|\eta_{t}-\eta_{t}^{0}\right|\left(\mathrm{d} y_{n+1}\right)\right] \\
& \leq \mathrm{E}\left[\int a\left(y_{n}, y_{n+1}\right)\left(\eta_{t}\left(\mathrm{~d} y_{n+1}\right)+\eta_{t}^{0}\left(\mathrm{~d} y_{n+1}\right)\right)\right] \\
& \leq 2 \mathrm{E}\left[\int a\left(y_{n}, y_{n+1}\right) \eta_{t}\left(\mathrm{~d} y_{n+1}\right)\right] \\
&= \mathrm{E}\left[\int_{0}^{t} \int_{\mathbb{R}^{d} \times[0,+\infty)^{2}} a\left(y_{n}, y_{n+1}\right) \mathbf{1}_{\left[0, \lambda\left(y_{n+1}, \eta_{s-}\right)\right]}(u) \mathbf{1}_{(t-s, \infty)}(r) N\left(\mathrm{~d} y_{n+1}, \mathrm{~d} s, \mathrm{~d} r, \mathrm{~d} u\right)\right] \\
&+\mathrm{E}\left[\int_{\mathbb{R}^{d} \times[0, \infty)} a\left(y_{n}, y_{n+1}\right) \mathbf{1}_{(t, \infty)}(r) \hat{\eta}_{0}\left(\mathrm{~d} y_{n+1}, \mathrm{~d} r\right)\right] \\
& \leq\left(L+\mu_{1}\right) \int a\left(y_{n}, y_{n+1}\right) \mathrm{d} y_{n+1} \\
& \leq\left(L+\mu_{1}\right) K,
\end{aligned}
$$

we have

$$
\begin{aligned}
R_{n}= & \int \cdots \int_{B_{0}} a(x, y) a\left(y, y_{1}\right) \cdots a\left(y_{n-1}, y_{n}\right) \\
& \times \int_{0}^{t} \mathrm{E}\left[\int a\left(y_{n}, y_{n+1}\right)\left|\eta_{t}-\eta_{t}^{0}\right|\left(\mathrm{d} y_{n+1}\right)\right] \frac{(t-s)^{n}}{n !} \mathrm{e}^{-(t-s)} \mathrm{d} s \mathrm{~d} y_{n+1} \mathrm{~d} y_{n} \cdots \mathrm{d} y_{1} \mathrm{~d} y \\
\leq & K \int_{0}^{t} \frac{(t-s)^{n}}{n !} \mathrm{e}^{-(t-s)} \mathrm{d} s \int \cdots \int a(x, y) a\left(y, y_{1}\right) \cdots a\left(y_{n-1}, y_{n}\right) \mathrm{d} y_{n} \cdots \mathrm{d} y_{1} \mathrm{~d} y \\
\leq & K^{n+2} \int_{0}^{t} \frac{(t-s)^{n}}{n !} \mathrm{e}^{-(t-s)} \mathrm{d} s \\
\rightarrow & 0 \text { as } n \rightarrow \infty .
\end{aligned}
$$

For any $0 \leq k \leq n$,

$$
\begin{aligned}
\int \cdots & \int_{B_{0}} a(x, y) a\left(y, y_{1}\right) \cdots a\left(y_{k-1}, y_{k}\right) \mathrm{d} y_{k} \cdots \mathrm{d} y_{1} \mathrm{~d} y \\
\leq & \int \cdots \int a(x, y) a\left(y, y_{1}\right) \cdots a\left(y_{k-2}, y_{k-1}\right) \frac{1}{c\left(y_{k-1}\right)} \\
& \times \int_{B_{0}} \frac{c\left(y_{k-1}\right) a\left(y_{k-1}, y_{k}\right)}{c\left(y_{k}\right)} \mathrm{d} y_{k} \sup _{z \in B_{0}} c(z) \mathrm{d} y_{k-1} \cdots \mathrm{d} y_{1} \mathrm{~d} y
\end{aligned}
$$




$$
\begin{aligned}
& \leq M\left[\sup _{z \in B_{0}} c(z)\right] \int \cdots \int a(x, y) a\left(y, y_{1}\right) \cdots a\left(y_{k-3}, y_{k-2}\right) \frac{1}{c\left(y_{k-2}\right)} \\
& \qquad \times \int \frac{c\left(y_{k-2}\right) a\left(y_{k-2}, y_{k-1}\right)}{c\left(y_{k-1}\right)} \mathrm{d} y_{k-1} \mathrm{~d} y_{k-2} \cdots \mathrm{d} y_{1} \mathrm{~d} y \\
& \leq \cdots \\
& \leq M^{k}\left[\sup _{z \in B_{0}} c(z)\right] \frac{1}{c(x)} \int \frac{c(x) a(x, y)}{c(y)} \mathrm{d} y \\
& \leq M^{k+1}\left[\sup _{z \in B_{0}} c(z)\right] \frac{1}{c(x)}
\end{aligned}
$$

and

$$
\int_{0}^{t} \frac{(t-s)^{k}}{k !} \mathrm{e}^{-(t-s)} \mathrm{d} s \leq \int_{0}^{t} \frac{(t-s)^{k}}{k !} \mathrm{d} s=\frac{t^{k+1}}{(k+1) !}
$$

Therefore,

$$
\mathrm{E}\left[\int a(x, y)\left|\eta_{t}-\eta_{t}^{0}\right|(\mathrm{d} y)\right] \leq 2\left(L+\mu_{1}\right) \sum_{k=1}^{n} \frac{(t M)^{k+1}}{(k+1) !}\left[\sup _{z \in B_{0}} c(z)\right] \frac{1}{c(x)}+R_{n},
$$

and, hence,

$$
\begin{aligned}
\mathrm{E}\left[\int a(x, y)\left|\eta_{t}-\eta_{t}^{0}\right|(\mathrm{d} y)\right] & \leq 2\left(L+\mu_{1}\right) \sum_{k=1}^{\infty} \frac{(t M)^{k+1}}{(k+1) !}\left[\sup _{z \in B_{0}} c(z)\right] \frac{1}{c(x)} \\
& \leq 2\left(L+\mu_{1}\right) \mathrm{e}^{t M}\left[\sup _{z \in B_{0}} c(z)\right] \frac{1}{c(x)}
\end{aligned}
$$

Now we have

$$
\sup _{x \in \mathbb{R}^{d}} c(x) \mathrm{E}\left[\int_{\mathbb{R}^{d}} a(x, y)\left|\eta_{t}-\eta_{t}^{0}\right|(\mathrm{d} y)\right] \leq 2\left(L+\mu_{1}\right)\left[\sup _{z \in B_{0}} c(z)\right] \mathrm{e}^{t M} .
$$

Condition 2.1 follows by the next lemma.

Lemma 4.2. Under the condition $\int_{\mathbb{R}^{d}}\left(1 / c(x)^{1 / 3}\right) \mathrm{d} x<\infty$, we have

$$
\sup \left\{\mathrm{E}\left[\left(\left|\eta_{t}-\eta_{t}^{0}\right|(B)\right)^{3}\right]: \text { B is a bounded measurable set in } \mathbb{R}^{d}\right\}<\infty .
$$

Proof. Fix $t \geq 0$. For $0 \leq t^{\prime} \leq t$ and any bounded measurable subset $B$, we define

$$
\begin{aligned}
\zeta_{t^{\prime}}(B)= & \int_{B \times\left[0, t^{\prime}\right] \times[0,+\infty)^{2}} \mathbf{1}_{\left[0, \lambda\left(x, \eta_{s-}\right)\right]}(u) \mathbf{1}_{(t-s, \infty)}(r) N(\mathrm{~d} x, \mathrm{~d} s, \mathrm{~d} r, \mathrm{~d} u) \\
& +\int_{B \times[0, \infty)} \mathbf{1}_{(t, \infty)}(r) \hat{\eta}_{0}(\mathrm{~d} x, \mathrm{~d} r) \\
\zeta_{t^{\prime}}^{0}(B)= & \int_{B \times\left[0, t^{\prime}\right] \times[0,+\infty)^{2}} \mathbf{1}_{\left[0, \lambda\left(x, \eta_{s-}^{0}\right)\right]}(u) \mathbf{1}_{(t-s, \infty)}(r) N^{0}(\mathrm{~d} x, \mathrm{~d} s, \mathrm{~d} r, \mathrm{~d} u) \\
& +\int_{B \times[0, \infty)} \mathbf{1}_{(t, \infty)}(r) \hat{\eta}_{0}^{0}(\mathrm{~d} x, \mathrm{~d} r) .
\end{aligned}
$$


Note that $\zeta_{t^{\prime}}(B)$ denotes the number of points in $B$ born before and at time $t^{\prime}$ that are still alive at time $t$. Let $Z_{t^{\prime}}=\left|\zeta_{t^{\prime}}-\zeta_{t^{\prime}}^{0}\right|(B)$. Then $Z_{t^{\prime}}$ is increasing and $Z_{t}=\left|\zeta_{t}-\zeta_{t}^{0}\right|(B)=\left|\eta_{t}-\eta_{t}^{0}\right|(B)$. Since the region $B$ is bounded, $Z_{t^{\prime}}$ has only finitely many jumps and $\Delta Z_{t^{\prime}}=0$ or 1 a.s. We have

$$
\begin{aligned}
Z_{t}^{3} & =\sum_{0<s \leq t}\left[Z_{s}^{3}-Z_{s-}^{3}\right]+Z_{0}^{3} \\
& =\sum_{0<s \leq t} 3 Z_{s-}^{2} \Delta Z_{s}+\sum_{0<s \leq t} 3 Z_{s-}\left(\Delta Z_{s}\right)^{2}+\sum_{0<s \leq t}\left(\Delta Z_{s}\right)^{3}+Z_{0}^{3} \\
& =\sum_{0<s \leq t} 3 Z_{s-}^{2} \Delta Z_{s}+\sum_{0<s \leq t} 3 Z_{s-} \Delta Z_{s}+\sum_{0<s \leq t} \Delta Z_{s}+Z_{0}^{3} .
\end{aligned}
$$

We have $Z_{0} \leq \eta_{0}\left(B_{0} \cap B\right)+\eta_{0}^{0}\left(B_{0} \cap B\right)$, and the right-hand side has a Poisson distribution with mean $2 \mu_{1} m\left(B_{0} \cap B\right)$, where $m$ is the Lebesgue measure. Hence, $\mathrm{E}\left[Z_{0}^{3}\right] \leq 8 \mu_{1}^{3} m\left(B_{0} \cap\right.$ $B)^{3}+12 \mu_{1}^{2} m\left(B_{0} \cap B\right)^{2}+2 \mu_{1} m\left(B_{0} \cap B\right) \leq 8 \mu_{1}^{3}+12 \mu_{1}^{2}+2 \mu_{1}$. Now we estimate the first term on the right-hand side of (4.2):

$$
\begin{aligned}
& \mathrm{E}\left[\sum_{0<s \leq t} 3 Z_{s-}^{2} \Delta Z_{s}\right] \\
& =3 \mathrm{E}\left[\int_{0}^{t} Z_{s-}^{2}\right. \\
& \times \int_{B \cap B_{0}^{\mathrm{c}} \times[0,+\infty)^{2}}\left|\mathbf{1}_{\left[0, \lambda\left(x, \eta_{s-}\right)\right]}(u)-\mathbf{1}_{\left[0, \lambda\left(x, \eta_{s-}^{0}\right)\right]}(u)\right| \\
& \left.\times \mathbf{1}_{(t-s, \infty)}(r) N(\mathrm{~d} x, \mathrm{~d} s, \mathrm{~d} r, \mathrm{~d} u)\right] \\
& +3 \mathrm{E}\left[\int_{0}^{t} Z_{s-}^{2} \int_{B \cap B_{0} \times[0,+\infty)^{2}} \mathbf{1}_{\left[0, \lambda\left(x, \eta_{s-}\right)\right]}(u) \mathbf{1}_{(t-s, \infty)}(r)\right] N(\mathrm{~d} x, \mathrm{~d} s, \mathrm{~d} r, \mathrm{~d} u) \\
& +3 \mathrm{E}\left[\int_{0}^{t} Z_{s-}^{2} \int_{B \cap B_{0} \times[0,+\infty)^{2}} \mathbf{1}_{\left[0, \lambda\left(x, \eta_{s-}^{0}\right)\right]}(u) \mathbf{1}_{(t-s, \infty)}(r) N_{0}(\mathrm{~d} x, \mathrm{~d} s, \mathrm{~d} r, \mathrm{~d} u)\right] \\
& =3 \mathrm{E}\left[\int_{0}^{t} Z_{s-}^{2}\right. \\
& \times \int_{B \cap B_{0}^{\mathrm{c}} \times[0,+\infty)^{2}}\left|\mathbf{1}_{\left[0, \lambda\left(x, \eta_{s-}\right)\right]}(u)-\mathbf{1}_{\left[0, \lambda\left(x, \eta_{s-}^{0}\right)\right]}(u)\right| \\
& \left.\times \mathbf{1}_{(t-s, \infty)}(r) \mathrm{d} x \mathrm{~d} s \mathrm{e}^{-r} \mathrm{~d} r \mathrm{~d} u\right] \\
& +3 \mathrm{E}\left[\int_{0}^{t} Z_{s-}^{2} \int_{B \cap B_{0} \times[0,+\infty)^{2}} \mathbf{1}_{\left[0, \lambda\left(x, \eta_{s-}\right)\right]}(u) \mathbf{1}_{(t-s, \infty)}(r) \mathrm{d} x \mathrm{~d} s \mathrm{e}^{-r} \mathrm{~d} r \mathrm{~d} u\right] \\
& +3 \mathrm{E}\left[\int_{0}^{t} Z_{s-}^{2} \int_{B \cap B_{0} \times[0,+\infty)^{2}} \mathbf{1}_{\left[0, \lambda\left(x, \eta_{s-}^{0}\right)\right]}(u) \mathbf{1}_{(t-s, \infty)}(r) \mathrm{d} x \mathrm{~d} s \mathrm{e}^{-r} \mathrm{~d} r \mathrm{~d} u\right] \\
& \leq 3 \mathrm{E}\left[\int_{0}^{t} Z_{s}^{2} \int_{B}\left|\lambda\left(x, \eta_{s}\right)-\lambda\left(x, \eta_{s}^{0}\right)\right| \mathrm{e}^{-(t-s)} \mathrm{d} x \mathrm{~d} s\right]+6 L \mathrm{E}\left[\int_{0}^{t} Z_{s}^{2} \mathrm{e}^{-(t-s)} \mathrm{d} s\right]
\end{aligned}
$$




$$
\begin{aligned}
& \leq 3 \int_{0}^{t} \int_{B} \mathrm{E}\left[Z_{t}^{2}\left|\lambda\left(x, \eta_{s}\right)-\lambda\left(x, \eta_{s}^{0}\right)\right|\right] \mathrm{e}^{-(t-s)} \mathrm{d} x \mathrm{~d} s+6 L \mathrm{E}\left[Z_{t}^{2}\right] \\
& \leq 3\left(\mathrm{E}\left[Z_{t}^{3}\right]\right)^{2 / 3} \int_{0}^{t} \int_{B}\left(\mathrm{E}\left|\lambda\left(x, \eta_{s}\right)-\lambda\left(x, \eta_{s}^{0}\right)\right|^{3}\right)^{1 / 3} \mathrm{e}^{-(t-s)} \mathrm{d} x \mathrm{~d} s+6 L\left(\mathrm{E}\left[Z_{t}^{3}\right]\right)^{2 / 3},
\end{aligned}
$$

Similarly, for the second term and the third term on the right-hand side of (4.2), we have

$$
\begin{aligned}
& 3 \mathrm{E}\left[\sum_{0 \leq s \leq t} 3 Z_{s-} \Delta Z_{s}\right] \\
& \quad \leq 3\left(\mathrm{E}\left[Z_{t}^{3}\right]\right)^{1 / 3} \int_{0}^{t} \int_{B}\left(\mathrm{E}\left|\lambda\left(x, \eta_{s}\right)-\lambda\left(x, \eta_{s}^{0}\right)\right|^{3 / 2}\right)^{2 / 3} \mathrm{e}^{-(t-s)} \mathrm{d} x \mathrm{~d} s+6 L\left(\mathrm{E}\left[Z_{t}^{3}\right]\right)^{1 / 3} \\
& \quad \leq 3\left(\mathrm{E}\left[Z_{t}^{3}\right]\right)^{1 / 3} \int_{0}^{t} \int_{B}\left(\mathrm{E}\left|\lambda\left(x, \eta_{s}\right)-\lambda\left(x, \eta_{s}^{0}\right)\right|^{3}\right)^{1 / 3} \mathrm{e}^{-(t-s)} \mathrm{d} x \mathrm{~d} s+6 L\left(\mathrm{E}\left[Z_{t}^{3}\right]\right)^{1 / 3}
\end{aligned}
$$

and

$$
\begin{aligned}
\mathrm{E}\left[\sum_{0 \leq s \leq t} \Delta Z_{s}\right] & \leq \int_{0}^{t} \int_{B} \mathrm{E}\left[\left|\lambda\left(x, \eta_{s}\right)-\lambda\left(x, \eta_{s}^{0}\right)\right|\right] \mathrm{e}^{-(t-s)} \mathrm{d} x \mathrm{~d} s+2 L \\
& \leq \int_{0}^{t} \int_{B}\left(\mathrm{E}\left|\lambda\left(x, \eta_{s}\right)-\lambda\left(x, \eta_{s}^{0}\right)\right|^{3}\right)^{1 / 3} \mathrm{e}^{-(t-s)} \mathrm{d} x \mathrm{~d} s+2 L .
\end{aligned}
$$

Now, we can estimate $\mathrm{E}\left[Z_{t}^{3}\right]$ :

$$
\begin{aligned}
\mathrm{E}\left[Z_{t}^{3}\right] \leq & 3\left(\mathrm{E}\left[Z_{t}^{3}\right]\right)^{2 / 3} \int_{0}^{t} \int_{B}\left(\mathrm{E}\left|\lambda\left(x, \eta_{s}\right)-\lambda\left(x, \eta_{s}^{0}\right)\right|^{3}\right)^{1 / 3} \mathrm{e}^{-(t-s)} \mathrm{d} x \mathrm{~d} s+6 L\left(\mathrm{E}\left[Z_{t}^{3}\right]\right)^{2 / 3} \\
& +3\left(\mathrm{E}\left[Z_{t}^{3}\right]\right)^{1 / 3} \int_{0}^{t} \int_{B}\left(\mathrm{E}\left|\lambda\left(x, \eta_{s}\right)-\lambda\left(x, \eta_{s}^{0}\right)\right|^{3}\right)^{1 / 3} \mathrm{e}^{-(t-s)} \mathrm{d} x \mathrm{~d} s+6 L\left(\mathrm{E}\left[Z_{t}^{3}\right]\right)^{1 / 3} \\
& +\int_{0}^{t} \int_{B}\left(\mathrm{E}\left|\lambda\left(x, \eta_{s}\right)-\lambda\left(x, \eta_{s}^{0}\right)\right|^{3}\right)^{1 / 3} \mathrm{e}^{-(t-s)} \mathrm{d} x \mathrm{~d} s+2 L+\mathrm{E}\left[Z_{0}^{3}\right] .
\end{aligned}
$$

Let $x=\left(\mathrm{E}\left[Z_{t}^{3}\right]\right)^{1 / 3}, a_{0}=b_{0}=3 \int_{0}^{t} \int_{B}\left(\mathrm{E}\left|\lambda\left(x, \eta_{s}\right)-\lambda\left(x, \eta_{s}^{0}\right)\right|^{3}\right)^{1 / 3} \mathrm{e}^{-(t-s)} \mathrm{d} x \mathrm{~d} s+6 L$, and $c_{0}=a_{0} / 3+\mathrm{E}\left[Z_{0}^{3}\right]$. Here $x, a_{0}, b_{0}$, and $c_{0}$ all depend on $B$. Now we have the inequality $x^{3} \leq a_{0} x^{2}+b_{0} x+c_{0}$. If we can show that $a_{0}, b_{0}$, and $c_{0}$ are all uniformly bounded for all $B$ then $x$ is uniformly bounded for all $B$, that is, we have Condition 2.2.

So we calculate

$$
\begin{aligned}
\int_{0}^{t} \int_{B}\left(\mathrm{E}\left|\lambda\left(x, \eta_{s}\right)-\lambda\left(x, \eta_{s}^{0}\right)\right|^{3}\right)^{1 / 3} \mathrm{e}^{-(t-s)} \mathrm{d} x \mathrm{~d} s \\
\quad \leq(2 L)^{2 / 3} \int_{0}^{t} \int_{B}\left(\mathrm{E}\left|\lambda\left(x, \eta_{s}\right)-\lambda\left(x, \eta_{s}^{0}\right)\right|\right)^{1 / 3} \mathrm{e}^{-(t-s)} \mathrm{d} x \mathrm{~d} s \\
\quad \leq(2 L)^{2 / 3} \int_{0}^{t} \int_{B}\left(\mathrm{E}\left[\int a(x, y)\left|\eta_{s}-\eta_{s}^{0}\right|(\mathrm{d} y)\right]\right)^{1 / 3} \mathrm{e}^{-(t-s)} \mathrm{d} x \mathrm{~d} s \\
\quad \leq(2 L)^{2 / 3} \int_{0}^{t} \int_{B}\left(\frac{1}{c(x)} \sup _{z} c(z) \mathrm{E}\left[\int a(z, y)\left|\eta_{s}-\eta_{s}^{0}\right|(\mathrm{d} y)\right]\right)^{1 / 3} \mathrm{e}^{-(t-s)} \mathrm{d} x \mathrm{~d} s \\
\quad \leq(2 L)^{2 / 3} \int_{B} \frac{1}{c(x)^{1 / 3}} \mathrm{~d} x \int_{0}^{t}\left(2\left(L+\mu_{1}\right)\left[\sup _{y \in B_{0}} c(y)\right] \mathrm{e}^{s M}\right)^{1 / 3} \mathrm{e}^{-(t-s)} \mathrm{d} s
\end{aligned}
$$




$$
\begin{aligned}
& \leq(2 L)^{2 / 3} \int_{B} \frac{1}{c(x)^{1 / 3}} \mathrm{~d} x \int_{0}^{t}\left(2\left(L+\mu_{1}\right) \sup _{y \in B_{0}} c(y)\right)^{1 / 3} \mathrm{e}^{s M / 3} \mathrm{e}^{-(t-s)} \mathrm{d} s \\
& \leq(2 L)^{2 / 3}\left(\int_{\mathbb{R}^{d}} \frac{1}{c(x)^{1 / 3}} \mathrm{~d} x\right) t\left(2\left(L+\mu_{1}\right) \sup _{y \in B_{0}} c(y)\right)^{1 / 3} \mathrm{e}^{t M / 3},
\end{aligned}
$$

which is uniformly bounded for all $B$, where the fourth inequality follows from Lemma 4.1. This completes the proof.

Next, we show that Condition 2.1 is satisfied. By a similar calculation as in the proof of Lemma 4.2, we have

$$
\begin{aligned}
\mathrm{E}\left[\left|\eta_{t}-\eta_{t}^{0}\right|(B)\right] \leq & \int_{0}^{t} \int_{B} \mathrm{E}\left[\left|\lambda\left(x, \eta_{s}\right)-\lambda\left(x, \eta_{s}^{0}\right)\right|\right] \mathrm{e}^{-(t-s)} \mathrm{d} x \mathrm{~d} s+2\left(L+\mu_{1}\right) \int_{B \cap B_{0}} \mathrm{~d} x \\
\leq & \int_{0}^{t} \int_{B} \mathrm{E}\left[\int a(x, y)\left|\eta_{s}-\eta_{s}^{0}\right|(\mathrm{d} y)\right] \mathrm{e}^{-(t-s)} \mathrm{d} s \mathrm{~d} x+2\left(L+\mu_{1}\right) \int_{B \cap B_{0}} \mathrm{~d} x \\
\leq & \int_{0}^{t} \int_{B} \frac{1}{c(x)} \sup _{z} c(z) \mathrm{E}\left[\int a(z, y)\left|\eta_{s}-\eta_{s}^{0}\right|(\mathrm{d} y)\right] \mathrm{e}^{-(t-s)} \mathrm{d} x \mathrm{~d} s \\
& +2\left(L+\mu_{1}\right) \int_{B \cap B_{0}} \mathrm{~d} x \\
\leq & J_{t}^{(1)}\left(\int_{B} \frac{1}{c(x)} \mathrm{d} x+\int_{B \cap B_{0}} \mathrm{~d} x\right)
\end{aligned}
$$

where $J_{t}^{(1)}$ is a constant which depends on $t$, but does not depend on $B$. By an argument similar to the proof of Lemma 4.2, we can show that $\left(\mathrm{E}\left[\left(\left|\eta_{t}-\eta_{t}^{0}\right|(B)\right)^{2}\right]\right)^{1 / 2}$ is less than a number $\alpha$ that satisfies the following inequality:

$$
\alpha^{2} \leq \alpha J_{t}^{\prime} \int_{B} \frac{1}{c(x)^{1 / 2}} \mathrm{~d} x+J_{t}^{\prime \prime}\left(\int_{B} \frac{1}{c(x)^{1 / 2}} \mathrm{~d} x+\int_{B \cap B_{0}} \mathrm{~d} x+\left(\int_{B \cap B_{0}} \mathrm{~d} x\right)^{2}\right) .
$$

Hence, we have

$\mathrm{E}\left[\left(\left|\eta_{t}-\eta_{t}^{0}\right|(B)\right)^{2}\right] \leq J_{t}^{(2)}\left(\left(\int_{B} \frac{1}{c(x)^{1 / 2}} \mathrm{~d} x\right)^{2}+\int_{B} \frac{1}{c(x)^{1 / 2}} \mathrm{~d} x+\int_{B \cap B_{0}} \mathrm{~d} x+\left(\int_{B \cap B_{0}} \mathrm{~d} x\right)^{2}\right)$,

where $J_{t}^{(1)}, J_{t}^{(2)}$, and $J_{t}^{\prime}, J_{t}^{\prime \prime}$ are all constants which depend on $t$, but do not depend on $B$.

Let $\left\{C_{n}\right\}$ be a sequence of bounded measurable subsets of $\mathbb{R}^{d}$ satisfying $\lim _{n \rightarrow \infty} C_{n}=$ $\mathbb{R}^{d}$. We will show that $\left\{\eta_{t}\left(C_{n}\right)-\eta_{t}^{0}\left(C_{n}\right) \mid n=1,2,3, \ldots\right\}$ is a Cauchy sequence in $L^{1}(\mathrm{P})$. For any two positive integers $m$ and $n$, by (4.3),

$$
\begin{aligned}
& \left|\mathrm{E}\left[\left(\eta_{t}\left(C_{n}\right)-\eta_{t}^{0}\left(C_{n}\right)\right)-\left(\eta_{t}\left(C_{m}\right)-\eta_{t}^{0}\left(C_{m}\right)\right)\right]\right| \\
& \quad \leq \mathrm{E}\left[\left|\eta_{t}-\eta_{t}^{0}\right|\left(C_{n} \backslash C_{m}\right)\right]+\mathrm{E}\left[\left|\eta_{t}-\eta_{t}^{0}\right|\left(C_{m} \backslash C_{n}\right)\right] \\
& \quad \leq J_{t}^{(1)}\left(\int_{C_{n} \Delta C_{m}} \frac{1}{c(x)} \mathrm{d} x+\int_{\left(C_{n} \Delta C_{m}\right) \cap B_{0}} \mathrm{~d} x\right),
\end{aligned}
$$

where $C_{n} \Delta C_{m}$ is the symmetric difference of $C_{n}$ and $C_{m}$ which converges to $\varnothing$ as $m, n \rightarrow \infty$. Therefore, $\left\{\eta_{t}\left(C_{n}\right)-\eta_{t}^{0}\left(C_{n}\right) \mid n=1,2,3, \ldots\right\}$ converges in $L^{1}(\mathrm{P})$. This proves the first 
stabilization condition by Remark 2.1(b). The second stabilization condition can be proved by the same argument.

Now we show that the third stabilization condition is true. Let $A$ be any given Riemann measurable subset in $\mathbb{R}^{d}$. Choose a large positive number $K_{A}$ such that $\sup _{x \in A}\|x\|<K_{A} / 2$. For any $l \geq 1$ and any $y \in \mathbb{Z}^{d}$ such that $\|y\| \geq l K_{A}$, we have $(l A+y) \cap\left\{x \in \mathbb{R}^{d}:\|x\| \leq\right.$ $\left.l K_{A} / 2\right\}=\varnothing$. Hence, for all large enough $l$, we have $(l A+y) \cap B_{0}=\varnothing$ and

$$
\int_{l A+y} \frac{1}{c(x)^{1 / 2}} \mathrm{~d} x \leq \int_{\|x\| \geq l K_{A} / 2} \frac{1}{c(x)^{1 / 2}} \mathrm{~d} x \leq 1 .
$$

By (4.4),

$$
\begin{aligned}
& \frac{1}{l^{d}} \sum_{\substack{y \in \mathbb{Z}^{d} \\
\|y\| \geq l K_{A}}} \mathrm{E}\left[\left(\Delta_{l, y}^{H}(A)\right)^{2}\right] \leq \frac{1}{l^{d}} \sum_{\substack{y \in \mathbb{Z}^{d} \\
\|y\| \geq l K_{A}}} \mathrm{E}\left[\left(\left|\eta_{t}-\eta_{t}^{0}\right|(l A+y)\right)^{2}\right] \\
& \leq \frac{1}{l^{d}} \sum_{\substack{y \in \mathbb{Z}^{d} \\
\|y\| \geq l K_{A}}} J_{t}^{(2)}\left(\left(\int_{l A+y} \frac{1}{c(x)^{1 / 2}} \mathrm{~d} x\right)^{2}+\int_{l A+y} \frac{1}{c(x)^{1 / 2}} \mathrm{~d} x\right. \\
& \left.+\int_{(l A+y) \cap B_{0}} \mathrm{~d} x+\left(\int_{(l A+y) \cap B_{0}} \mathrm{~d} x\right)^{2}\right) \\
& \leq \frac{1}{l^{d}} \sum_{\substack{y \in \mathbb{Z}^{d} \\
\|y\| \geq l K_{A}}} J_{t}^{(2)}\left(\int_{l A+y} \frac{1}{c(x)^{1 / 2}} \mathrm{~d} x+\int_{l A+y} \frac{1}{c(x)^{1 / 2}} \mathrm{~d} x\right) \\
& \leq \frac{2}{l^{d}} \sum_{\substack{y \in \mathbb{Z}^{d} \\
\|y\| \geq l K_{A}}} J_{t}^{(2)} \int_{l A+y} \frac{1}{c(x)^{1 / 2}} \mathrm{~d} x \\
& \leq \frac{2 J_{t}^{(2)}}{l^{d}} \sum_{\substack{y \in \mathbb{Z}^{d} \\
\|y\| \geq l K_{A}}} \sum_{\substack{z \in \mathbb{Z}^{d} \\
\left(z+B_{0}\right) \cap l A \neq \varnothing}} \int_{B_{0}+z+y} \frac{1}{c(x)^{1 / 2}} \mathrm{~d} x \\
& =\frac{2 J_{t}^{(2)}}{l^{d}} \sum_{\substack{z \in \mathbb{Z}^{d} \\
\left(z+B_{0}\right) \cap l A \neq \varnothing}} \sum_{\substack{y \in \mathbb{Z}^{d} \\
\|y\| \geq l K_{A}}} \int_{B_{0}+z+y} \frac{1}{c(x)^{1 / 2}} \mathrm{~d} x \\
& \leq \frac{2 J_{t}^{(2)}}{l^{d}} \sum_{\substack{z \in \mathbb{Z}^{d} \\
\left(z+B_{0}\right) \cap l A \neq \varnothing}} \int_{\|x\| \geq l K_{A} / 2-2} \frac{1}{c(x)^{1 / 2}} \mathrm{~d} x \\
& \leq \frac{2 J_{t}^{(2)}}{l^{d}}\left(l K_{A}+2\right)^{d} \int_{\|x\| \geq l K_{A} / 2-2} \frac{1}{c(x)^{1 / 2}} \mathrm{~d} x \\
& \rightarrow 0 \text { as } l \rightarrow \infty \text {, }
\end{aligned}
$$

where the penultimate inequality is true because, for any $x \in B_{0}+z+y,\|x\| \geq\|y\|-\|z\|-1 \geq$ $l K_{A} / 2-2$. Hence, the third stabilization condition is true. Now we can derive the following theorem from Theorem 2.1. 
Theorem 4.2. Assume that the birth rate $\lambda$ is bounded by some positive number $L$ and that it is translation invariant. Suppose that $\eta_{0}$ is a Poisson random measure on $\mathbb{R}^{d}$ with constant intensity and that there exists a positive function $c(x)$ such that (2.6) is satisfied. The function $c(x)$ is bounded in a neighborhood of the origin in $\mathbb{R}^{d}$, i.e. $\int_{\mathbb{R}^{d}}(1 / c(x)) \mathrm{d} x<\infty$ and $\int_{\mathbb{R}^{d}}\left(1 / c(x)^{1 / 3}\right) \mathrm{d} x<\infty$. Suppose that

$$
\sup _{x} \int_{\mathbb{R}^{d}} a(x, y) \mathrm{d} y<\infty .
$$

Let $\xi_{t}^{(l)}(A)=\left(1 / l^{d / 2}\right)\left(\eta_{t}(l A)-\mathrm{E}\left[\eta_{t}(l A)\right]\right)$, where $1 \leq l<\infty$. For any $0 \leq t_{1} \leq \cdots \leq t_{n}$ and Riemann measurable sets $A_{1}, \ldots, A_{n},\left(\xi_{t_{1}}^{(l)}\left(A_{1}\right), \ldots, \xi_{t_{n}}^{(l)}\left(A_{n}\right)\right)$ converges weakly to $a$ multivariate normal distribution and the variance-covariance matrix of the limit distribution can be given in terms of $\Delta_{\infty}$, as in Theorem 2.1.

Recall that we defined $Y^{(l)}, l \geq 1$, as

$$
Y^{(l)}(f, t)=\frac{1}{l^{d / 2}}\left(\int_{\mathbb{R}^{d}} f\left(\frac{x}{l}\right) \eta_{t}(\mathrm{~d} x)-\mathrm{E}\left[\int_{\mathbb{R}^{d}} f\left(\frac{x}{l}\right) \eta_{t}(\mathrm{~d} x)\right]\right)
$$

for $t \geq 0$ and $f \in L^{1}\left(\mathbb{R}^{d}\right) \cap L^{\infty}\left(\mathbb{R}^{d}\right)$.

We will show, by using Theorem 8.6 of Ethier and Kurtz (1985), that, for any sequence $\left\{l_{n}\right\}$ such that $l_{n} \rightarrow \infty$ as $n \rightarrow \infty$, the sequence $\left\{Y^{\left(l_{n}\right)}(f, \cdot): n \geq 1\right\}$ is relatively compact in $D_{\mathbb{R}}[0, \infty)$. Let $\mathcal{F}_{t}$ be the $\sigma$-field generated by $\left.N\right|_{\mathbb{R}^{d} \times[0, t] \times[0, \infty)^{2}}$ and $\left.\hat{\eta}_{0}\right|_{\mathbb{R}^{d} \times[0, t]}$.

Lemma 4.3.

$$
\mathrm{e}^{t} \int_{\mathbb{R}^{d} \times[0, \infty)} f\left(\frac{x}{l}\right) \mathbf{1}_{(t, \infty)}(r) \hat{\eta}_{0}(\mathrm{~d} x, \mathrm{~d} r), \quad t \geq 0,
$$

is an $\left(\mathcal{F}_{t}\right)$-martingale for any $l \geq 1$. Its quadratic variation is

$$
\int_{\mathbb{R}^{d} \times[0, \infty)} \mathrm{e}^{2 r} f^{2}\left(\frac{x}{l}\right) \mathbf{1}_{[0, t]}(r) \hat{\eta}_{0}(\mathrm{~d} x, \mathrm{~d} r), \quad t \geq 0 .
$$

Proof. Let $\left\{\tau_{x}: x \in \mathbb{R}^{d}\right\}$ be a family of independent unit exponentials, independent of $\eta_{0}$ and $N$. Then $\hat{\eta}_{0}=\sum_{x \in \eta_{0}} \delta_{\left(x, \tau_{x}\right)}$. Let $Z_{t}^{x}=\mathbf{1}_{\left[\tau_{x}>t\right]}$. Then

$$
\mathrm{e}^{t} \int_{\mathbb{R}^{d} \times[0, \infty)} f\left(\frac{x}{l}\right) \mathbf{1}_{(t, \infty)}(r) \hat{\eta}_{0}(\mathrm{~d} x, \mathrm{~d} r)=\sum_{x \in \eta_{0}} f\left(\frac{x}{l}\right) \mathrm{e}^{t} Z_{t}^{x} .
$$

Because $\int_{\mathbb{R}^{d}}|f(x / l)| \eta_{0}(\mathrm{~d} x)$ is an integrable random variable, the series on the right-hand side is absolutely convergent. On the other hand, we have

$$
\mathcal{F}_{t} \subseteq \sigma\left\{\left.N\right|_{\mathbb{R}^{d} \times[0, t] \times[0, \infty)^{2}}\right\} \vee \sigma\left\{\eta_{0}\right\} \vee \sigma\left\{Z_{r}^{x}: r \leq t, x \in \mathbb{R}^{d}\right\} .
$$

It is easy to check that $\mathrm{e}^{t} Z_{t}^{x}$ is a $\sigma\left\{Z_{r}^{x}: r \leq t\right\}$-martingale and, hence, that it is a $\sigma\left\{Z_{r}^{x}: r \leq\right.$ $\left.t, x \in \mathbb{R}^{d}\right\}$-martingale. Let $\mathcal{M}_{t}$ denote $\sigma\left\{Z_{r}^{x}: r \leq t, x \in \mathbb{R}^{d}\right\}$. Let $0 \leq s<t$. Since $\sigma\left\{\eta_{0}\right\}$ and $\mathcal{M}_{s}$ are independent, for any $E \in \sigma\left\{\eta_{0}\right\}$ and $F \in \mathcal{M}_{s}$, we have

$$
\begin{aligned}
\int_{E \cap F} \sum_{x \in \eta_{0}} f\left(\frac{x}{l}\right) \mathrm{e}^{t} Z_{t}^{x} \mathrm{dP} & \left.=\int_{E \cap F} \sum_{x \in \eta_{0}} f\left(\frac{x}{l}\right) \mathrm{E}^{t} \mathrm{e}^{t} Z_{t}^{x} \mid \mathcal{M}_{s}\right] \mathrm{dP} \\
& =\int_{E \cap F} \sum_{x \in \eta_{0}} f\left(\frac{x}{l}\right) \mathrm{e}^{s} Z_{s}^{x} \mathrm{dP} .
\end{aligned}
$$


By the $\pi-\lambda$ theorem,

$$
\mathrm{E}\left[\sum_{x \in \eta_{0}} f\left(\frac{x}{l}\right) \mathrm{e}^{t} Z_{t}^{x} \mid \sigma\left\{\eta_{0}\right\} \vee \sigma\left\{Z_{r}^{x}: r \leq t, x \in \mathbb{R}^{d}\right\}\right]=\sum_{x \in \eta_{0}} f\left(\frac{x}{l}\right) \mathrm{e}^{s} Z_{s}^{x} .
$$

Since $\sigma\left\{\left.N\right|_{\mathbb{R}^{d} \times[0, t] \times[0, \infty)^{2}}\right\}, \sigma\left\{\eta_{0}\right\}$, and $\sigma\left\{Z_{r}^{x}: r \leq t, x \in \mathbb{R}^{d}\right\}$ are independent, we have

$$
\begin{gathered}
\mathrm{E}\left[\sum_{x \in \eta_{0}} f\left(\frac{x}{l}\right) \mathrm{e}^{t} Z_{t}^{x} \mid \sigma\left\{\left.N\right|_{\left.\left.\mathbb{R}^{d} \times[0, t] \times[0, \infty)^{2}\right\} \vee \sigma\left\{\eta_{0}\right\} \vee \sigma\left\{Z_{r}^{x}: r \leq t, x \in \mathbb{R}^{d}\right\}\right]}\right.\right. \\
=\mathrm{E}\left[\sum_{x \in \eta_{0}} f\left(\frac{x}{l}\right) \mathrm{e}^{t} Z_{t}^{x} \mid \sigma\left\{\eta_{0}\right\} \vee \sigma\left\{Z_{r}^{x}: r \leq t, x \in \mathbb{R}^{d}\right\}\right] \\
=\sum_{x \in \eta_{0}} f\left(\frac{x}{l}\right) \mathrm{e}^{s} Z_{s}^{x} .
\end{gathered}
$$

Hence,

$$
\begin{aligned}
& \mathrm{E}\left[\sum_{x \in \eta_{0}} f\left(\frac{x}{l}\right) \mathrm{e}^{t} Z_{t}^{x} \mid \mathcal{F}_{s}\right] \\
& =\mathrm{E}\left[\mathrm { E } \left[\sum_{x \in \eta_{0}} f\left(\frac{x}{l}\right) \mathrm{e}^{t} Z_{t}^{x} \mid \sigma\left\{\left.N\right|_{\left.\mathbb{R}^{d} \times[0, t] \times[0, \infty)^{2}\right\} \vee \sigma\left\{\eta_{0}\right\}}\right.\right.\right. \\
& \left.\left.\qquad \vee \sigma\left\{Z_{r}^{x}: r \leq t, x \in \mathbb{R}^{d}\right\}\right] \mid \mathcal{F}_{s}\right] \\
& =\mathrm{E}\left[\sum_{x \in \eta_{0}} f\left(\frac{x}{l}\right) \mathrm{e}^{s} Z_{s}^{x} \mid \mathcal{F}_{s}\right] \\
& =\sum_{x \in \eta_{0}} f\left(\frac{x}{l}\right) \mathrm{e}^{s} Z_{s}^{x} .
\end{aligned}
$$

Now we have proved that $\mathrm{e}^{t} \int_{\mathbb{R}^{d} \times[0, \infty)} f(x / l) \mathbf{1}_{(t, \infty)}(r) \hat{\eta}_{0}(\mathrm{~d} x, \mathrm{~d} r)$ is an $\mathcal{F}_{t}$-martingale. Furthermore, it is a purely discontinuous martingale, so its quadratic variation is just the sum of its squared jumps.

Lemma 4.4. Suppose that $\lambda$ is bounded by a constant $L$ and that

$$
a(x, y) \leq \frac{b}{1+|x-y|^{2 d+\delta}}
$$

for some constants $b>0$ and $\delta>0$. Then

$$
\sup _{\substack{x, y \in \mathbb{R}^{d} \\ s, t \leq T}}\left(1 \vee|x-y|^{d+\delta}\right)\left|\mathrm{E}\left[\left(\lambda\left(x, \eta_{s}\right)-\mathrm{E}\left[\lambda\left(x, \eta_{s}\right)\right]\right)\left(\lambda\left(y, \eta_{t}\right)-\mathrm{E}\left[\lambda\left(y, \eta_{t}\right)\right]\right)\right]\right|<\infty
$$

for any $T>0$, where $1 \vee|x-y|^{d+\delta}$ denotes $\max \left\{1,|x-y|^{d+\delta}\right\}$.

Proof. Let $x \neq y \in \mathbb{R}^{d}$, and let

$$
C_{x y}=\left\{z \in \mathbb{R}^{d}:|z-x|<|z-y|\right\} .
$$


Then $C_{x y}$ is a half-space in $\mathbb{R}^{d}$, which is closer to $x$ than to $y$. Similarly, we define $C_{y x}$, the half-space in $\mathbb{R}^{d}$, which is closer to $y$ than to $x$. Let $U_{x}$ and $U_{y}$ be the $\sigma$-fields generated by the restriction of $N$ to $C_{x y} \times[0, \infty)^{3}$ and $C_{y x} \times[0, \infty)^{3}$, respectively, and the restriction of $\hat{\eta}_{0}$ to $C_{x y} \times[0, \infty)$ and $C_{y x} \times[0, \infty)$, respectively. Let $\eta_{t}^{\prime}$ be the unique solution of the stochastic differential equation (4.1) with $N$ replaced by another Poisson random measure $N^{\prime}$, which is obtained by replacing $\left.N\right|_{C_{x y}^{c} \times[0, \infty)^{3}}$ by an independent copy and keeping $\left.N\right|_{C_{x y} \times[0, \infty)^{3}}$ the same, and with $\hat{\eta}_{0}$ replaced by $\hat{\eta}_{0}^{\prime}$, which is obtained by replacing $\left.\hat{\eta}_{0}\right|_{C_{x y}^{c} \times[0, \infty)}$ by an independent copy and keeping $\left.\hat{\eta}_{0}\right|_{C_{x y} \times[0, \infty)}$ the same. Let $\eta_{t}^{\prime \prime}$ be the unique solution of the stochastic differential equation (4.1) with $N$ replaced by another Poisson random measure $N^{\prime \prime}$, which is obtained by replacing $\left.N\right|_{C_{y x}^{c} \times[0, \infty)^{3}}$ by an independent copy and keeping the restriction of $N$ to $C_{y x} \times[0, \infty)^{3}$ the same, and with $\hat{\eta}_{0}$ replaced by $\hat{\eta}_{0}^{\prime \prime}$, which is obtained by replacing $\left.\hat{\eta}_{0}\right|_{C_{y x}^{\mathrm{c}} \times[0, \infty)}$ by an independent copy and keeping the restriction of $\hat{\eta}_{0}$ to $C_{y x} \times[0, \infty)$ the same. Then we have

$$
\begin{aligned}
& \mathrm{E}\left[\left(\lambda\left(x, \eta_{s}\right)-\mathrm{E}\left[\lambda\left(x, \eta_{s}\right)\right]\right)\left(\lambda\left(y, \eta_{t}\right)-\mathrm{E}\left[\lambda\left(y, \eta_{t}\right)\right]\right)\right] \\
&= \mathrm{E}\left[\left(\lambda\left(x, \eta_{s}\right)-\mathrm{E}\left[\lambda\left(x, \eta_{s}\right) \mid u_{x}\right]+\mathrm{E}\left[\lambda\left(x, \eta_{s}\right) \mid u_{x}\right]\right.\right. \\
&\left.\left.-\mathrm{E}\left[\lambda\left(x, \eta_{s}\right)\right]\right)\left(\lambda\left(y, \eta_{t}\right)-\mathrm{E}\left[\lambda\left(y, \eta_{t}\right)\right]\right)\right] \\
&= \mathrm{E}\left[\left(\lambda\left(x, \eta_{s}\right)-\mathrm{E}\left[\lambda\left(x, \eta_{s}\right) \mid u_{x}\right]\right)\left(\lambda\left(y, \eta_{t}\right)-\mathrm{E}\left[\lambda\left(y, \eta_{t}\right)\right]\right)\right] \\
&+\mathrm{E}\left[\left(\mathrm{E}\left[\lambda\left(x, \eta_{s}\right) \mid u_{x}\right]-\mathrm{E}\left[\lambda\left(x, \eta_{s}\right)\right]\right)\left(\lambda\left(y, \eta_{t}\right)-\mathrm{E}\left[\lambda\left(y, \eta_{t}\right)\right]\right)\right] \\
&= \mathrm{E}\left[\left(\lambda\left(x, \eta_{s}\right)-\mathrm{E}\left[\lambda\left(x, \eta_{s}\right) \mid u_{x}\right]\right)\left(\lambda\left(y, \eta_{t}\right)-\mathrm{E}\left[\lambda\left(y, \eta_{t}\right)\right]\right)\right] \\
&+\mathrm{E}\left[\mathrm{E}\left[\left(\mathrm{E}\left[\lambda\left(x, \eta_{s}\right) \mid u_{x}\right]-\mathrm{E}\left[\lambda\left(x, \eta_{s}\right)\right]\right)\left(\lambda\left(y, \eta_{t}\right)-\mathrm{E}\left[\lambda\left(y, \eta_{t}\right)\right]\right) \mid u_{x}\right]\right] \\
&= \mathrm{E}\left[\left(\lambda\left(x, \eta_{s}\right)-\mathrm{E}\left[\lambda\left(x, \eta_{s}\right) \mid u_{x}\right]\right)\left(\lambda\left(y, \eta_{t}\right)-\mathrm{E}\left[\lambda\left(y, \eta_{t}\right)\right]\right)\right] \\
&+\mathrm{E}\left[\left(\mathrm{E}\left[\lambda\left(x, \eta_{s}\right) \mid u_{x}\right]-\mathrm{E}\left[\lambda\left(x, \eta_{s}\right)\right]\right) \mathrm{E}\left[\left(\lambda\left(y, \eta_{t}\right)-\mathrm{E}\left[\lambda\left(y, \eta_{t}\right)\right]\right) \mid u_{x}\right]\right] \\
&= \mathrm{E}\left[\left(\lambda\left(x, \eta_{s}\right)-\mathrm{E}\left[\lambda\left(x, \eta_{s}\right) \mid u_{x}\right]\right)\left(\lambda\left(y, \eta_{t}\right)-\mathrm{E}\left[\lambda\left(y, \eta_{t}\right)\right]\right)\right] \\
&+\mathrm{E}\left[\left(\mathrm{E}\left[\lambda\left(x, \eta_{s}\right) \mid u_{x}\right]-\mathrm{E}\left[\lambda\left(x, \eta_{s}\right)\right]\right) \mathrm{E}\left[\left(\lambda\left(y, \eta_{t}\right)-\mathrm{E}\left[\lambda\left(y, \eta_{t}\right) \mid u_{y}\right]\right) \mid u\right]\right] \\
&= \mathrm{E}\left[\left(\lambda\left(x, \eta_{s}\right)-\mathrm{E}\left[\lambda\left(x, \eta_{s}\right) \mid u_{x}\right]\right)\left(\lambda\left(y, \eta_{t}\right)-\mathrm{E}\left[\lambda\left(y, \eta_{t}\right)\right]\right)\right] \\
&+\mathrm{E}\left[\mathrm{E}\left[\left(\mathrm{E}\left[\lambda\left(x, \eta_{s}\right) \mid u_{x}\right]-\mathrm{E}\left[\lambda\left(x, \eta_{s}\right)\right]\right)\left(\lambda\left(y, \eta_{t}\right)-\mathrm{E}\left[\lambda\left(y, \eta_{t}\right) \mid u_{y}\right]\right) \mid u_{x}\right]\right] \\
&= \mathrm{E}\left[\left(\lambda\left(x, \eta_{s}\right)-\mathrm{E}\left[\lambda\left(x, \eta_{s}\right) \mid u_{x}\right]\right)\left(\lambda\left(y, \eta_{t}\right)-\mathrm{E}\left[\lambda\left(y, \eta_{t}\right)\right]\right)\right] \\
&+\mathrm{E}\left[\left(\mathrm{E}\left[\lambda\left(x, \eta_{s}\right) \mid u_{x}\right]-\mathrm{E}\left[\lambda\left(x, \eta_{s}\right)\right]\right)\left(\lambda\left(y, \eta_{t}\right)-\mathrm{E}\left[\lambda\left(y, \eta_{t}\right) \mid u_{y}\right]\right)\right] \\
&= \mathrm{E}\left[\left(\lambda\left(x, \eta_{s}\right)-\lambda\left(x, \eta_{s}^{\prime}\right)\right)\left(\lambda\left(y, \eta_{t}\right)-\mathrm{E}\left[\lambda\left(y, \eta_{t}\right)\right]\right)\right] \\
&+\mathrm{E}\left[\left(\mathrm{E}\left[\lambda\left(x, \eta_{s}\right) \mid u_{x}\right]-\mathrm{E}\left[\lambda\left(x, \eta_{s}\right)\right]\right)\left(\lambda\left(y, \eta_{t}\right)-\lambda\left(y, \eta_{t}^{\prime \prime}\right)\right)\right],
\end{aligned}
$$

where the fifth equality is true because $U_{y}$ and $u_{x}$ are independent, hence,

$$
\mathrm{E}\left[\mathrm{E}\left[\lambda\left(y, \eta_{t}\right) \mid \mathcal{u}_{y}\right] \mid \mathcal{u}_{x}\right]=\mathrm{E}\left[\lambda\left(y, \eta_{t}\right)\right] .
$$

Therefore,

$$
\begin{aligned}
\left|\mathrm{E}\left[\left(\lambda\left(x, \eta_{s}\right)-\mathrm{E}\left[\lambda\left(x, \eta_{s}\right)\right]\right)\left(\lambda\left(y, \eta_{t}\right)-\mathrm{E}\left[\lambda\left(y, \eta_{t}\right)\right]\right)\right]\right| \\
\quad=\left|\mathrm{E}\left[\left(\lambda\left(x, \eta_{s}\right)-\lambda\left(x, \eta_{s}^{\prime}\right)\right)\left(\lambda\left(y, \eta_{t}\right)-\mathrm{E}\left[\lambda\left(y, \eta_{t}\right)\right]\right)\right]\right| \\
\quad+\left|\mathrm{E}\left[\left(\mathrm{E}\left[\lambda\left(x, \eta_{s}\right) \mid \mathcal{u}_{x}\right]-\mathrm{E}\left[\lambda\left(x, \eta_{s}\right)\right]\right)\left(\lambda\left(y, \eta_{t}\right)-\lambda\left(y, \eta_{t}^{\prime \prime}\right)\right)\right]\right| \\
\quad \leq 2 L \mathrm{E}\left[\left|\lambda\left(x, \eta_{s}\right)-\lambda\left(x, \eta_{s}^{\prime}\right)\right|\right]+2 L \mathrm{E}\left[\left|\lambda\left(y, \eta_{t}\right)-\lambda\left(y, \eta_{t}^{\prime \prime}\right)\right|\right] .
\end{aligned}
$$

Define

$$
f(z, s)=\mathrm{E}\left[\left|\lambda\left(z, \eta_{s}\right)-\lambda\left(z, \eta_{s}^{\prime}\right)\right|\right]
$$


Note that $|f(z, s)| \leq L$ for all $(z, s)$ pairs. Then we have

$$
\begin{aligned}
& f(z, s)=\mathrm{E}\left[\left|\lambda\left(z, \eta_{s}\right)-\lambda\left(z, \eta_{s}^{\prime}\right)\right|\right] \\
& \leq \mathrm{E}\left[\int_{\mathbb{R}^{d}} a\left(z, z_{1}\right)\left|\eta_{s}-\eta_{s}^{\prime}\right|\left(\mathrm{d} z_{1}\right)\right] \\
& =\mathrm{E}\left[\int_{0}^{s} \int_{C_{x y} \times[0, \infty)^{2}} a\left(z, z_{1}\right)\left|\mathbf{1}_{\left[0, \lambda\left(z, \eta_{s_{1}}\right)\right]}(u)-\mathbf{1}_{\left[0, \lambda\left(z, \eta_{s_{1}}^{\prime}\right)\right]}(u)\right|\right. \\
& \left.\times \mathbf{1}_{\left(s-s_{1}, \infty\right)}(r) \mathrm{d} z_{1} \mathrm{~d} s_{1} \mathrm{e}^{-r} \mathrm{~d} r \mathrm{~d} u\right] \\
& +\mathrm{E}\left[\int _ { 0 } ^ { s } \int _ { C _ { x y } ^ { \mathrm { c } } \times [ 0 , \infty ) ^ { 2 } } a ( z , z _ { 1 } ) \left(\mathbf{1}_{\left[0, \lambda\left(z, \eta_{s_{1}}\right)\right]}(u)\right.\right. \\
& \left.\left.+\mathbf{1}_{\left[0, \lambda\left(z, \eta_{s_{1}}^{\prime}\right)\right]}(u)\right) \mathbf{1}_{\left(s-s_{1}, \infty\right)}(r) \mathrm{d} z_{1} \mathrm{~d} s_{1} \mathrm{e}^{-r} \mathrm{~d} r \mathrm{~d} u\right] \\
& +\mathrm{E}\left[\int_{C_{x y}^{\mathrm{c}} \times[0, \infty)} a\left(z, z_{1}\right) \mathbf{1}_{(s, \infty)}(r) \hat{\eta}_{0}(\mathrm{~d} x, \mathrm{~d} r)\right] \\
& +\mathrm{E}\left[\int_{C_{x y}^{\mathrm{c}} \times[0, \infty)} a\left(z, z_{1}\right) \mathbf{1}_{(s, \infty)}(r) \hat{\eta}_{0}^{\prime}(\mathrm{d} x, \mathrm{~d} r)\right] \\
& =\mathrm{E}\left[\int_{0}^{s} \int_{C_{x y}} a\left(z, z_{1}\right)\left|\lambda\left(z, \eta_{s_{1}}\right)-\lambda\left(z, \eta_{s_{1}}^{\prime}\right)\right| \exp \left(-\left(s-s_{1}\right)\right) \mathrm{d} z_{1} \mathrm{~d} s_{1}\right] \\
& +\mathrm{E}\left[\int_{0}^{s} \int_{C_{x y}^{\mathrm{c}}} a\left(z, z_{1}\right)\left(\lambda\left(z, \eta_{s_{1}}\right)+\lambda\left(z, \eta_{s_{1}}^{\prime}\right)\right) \exp \left(-\left(s-s_{1}\right)\right) \mathrm{d} z_{1} \mathrm{~d} s_{1}\right] \\
& +\mathrm{E}\left[\int_{C_{x y}^{\mathrm{c}} \times[0, \infty)} a\left(z, z_{1}\right) \mathbf{1}_{(s, \infty)}(r) \hat{\eta}_{0}\left(\mathrm{~d} z_{1}, \mathrm{~d} r\right)\right] \\
& +\mathrm{E}\left[\int_{C_{x y}^{\mathrm{c}} \times[0, \infty)} a\left(z, z_{1}\right) \mathbf{1}_{(s, \infty)}(r) \hat{\eta}_{0}^{\prime}\left(\mathrm{d} z_{1}, \mathrm{~d} r\right)\right] \\
& \leq \mathrm{E}\left[\int_{0}^{s} \int_{C_{x y}} a\left(z, z_{1}\right)\left|\lambda\left(z, \eta_{s_{1}}\right)-\lambda\left(z, \eta_{s_{1}}^{\prime}\right)\right| \exp \left(-\left(s-s_{1}\right)\right) \mathrm{d} z_{1} \mathrm{~d} s_{1}\right] \\
& +2 L \int_{C_{x y}^{\mathrm{c}}} a\left(z, z_{1}\right) \mathrm{d} z_{1}+\mathrm{E}\left[\int_{C_{x y}^{\mathrm{c}}} a\left(z, z_{1}\right) \eta_{0}\left(\mathrm{~d} z_{1}\right)\right]+\mathrm{E}\left[\int_{C_{x y}^{\mathrm{c}}} a\left(z, z_{1}\right) \eta_{0}^{\prime}\left(\mathrm{d} z_{1}\right)\right] \\
& =2\left(L+\mu_{1}\right) \int_{C_{x y}^{\mathrm{c}}} a\left(z, z_{1}\right) \mathrm{d} z_{1}+\int_{0}^{s} \int_{C_{x y}} a\left(z, z_{1}\right) f\left(z_{1}, s_{1}\right) \exp \left(-\left(s-s_{1}\right)\right) \mathrm{d} z_{1} \mathrm{~d} s_{1} .
\end{aligned}
$$

By iteration, we obtain

$$
\begin{aligned}
& f(x, s)=\mathrm{E}\left[\left|\lambda\left(x, \eta_{s}\right)-\lambda\left(x, \hat{\eta}_{s}^{\prime}\right)\right|\right] \\
& \leq 2\left(L+\mu_{1}\right)\left(\int_{C_{x y}^{\mathrm{c}}} a\left(x, z_{1}\right) \mathrm{d} z_{1}\right.+\int_{C_{x y}} \int_{C_{x y}^{\mathrm{c}}} a\left(x, z_{1}\right) a\left(z_{1}, z_{2}\right) \mathrm{d} z_{2} \mathrm{~d} z_{1} \\
&+\frac{1}{(n-1) !} \int_{C_{x y}} \cdots \int_{C_{x y}} \int_{C_{x y}^{\mathrm{c}}} a\left(x, z_{1}\right) \cdots a\left(z_{n-2}, z_{n-1}\right) \\
&\left.\times a\left(z_{n-1}, z_{n}\right) \mathrm{d} z_{n} \mathrm{~d} z_{n-1} \cdots \mathrm{d} z_{1}\right)+R_{n},
\end{aligned}
$$


where the reminder term

$$
\begin{aligned}
R_{n} & =\frac{1}{(n-1) !} \int_{0}^{s} \int_{C_{x y}} \cdots \int_{C_{x y}} a\left(x, z_{1}\right) \cdots a\left(z_{n-1}, z_{n}\right) f\left(z_{n}, s_{n}\right) \exp \left(-\left(s-s_{n}\right)\right) \\
& \times\left(s-s_{n}\right)^{n-1} \mathrm{~d} z_{n} \mathrm{~d} z_{n-1} \cdots \mathrm{d} z_{1} \mathrm{~d} s_{n} \\
& \leq \frac{1}{(n-1) !}\left(\sup _{z} \int_{\mathbb{R}^{d}} a\left(z, z_{1}\right) \mathrm{d} z_{1}\right)^{n} L \int_{0}^{s} \exp \left(-\left(s-s_{n}\right)\right)\left(s-s_{n}\right)^{n-1} \mathrm{~d} s_{n} \\
& \leq \frac{1}{(n-1) !}\left(\int_{\mathbb{R}^{d}} \frac{b}{1+|x|^{2 d+\delta}} \mathrm{d} x\right)^{n} L \int_{0}^{s} \exp \left(-\left(s-s_{n}\right)\right)\left(s-s_{n}\right)^{n-1} \mathrm{~d} s_{n} \\
& \rightarrow 0 \text { as } n \rightarrow \infty
\end{aligned}
$$

Now we estimate the other terms in (4.5). In order to simplify the notation, we just calculate the term for $n=3$. Let $K_{n}=|x-y| / 2 n$, and recall that we have defined $K=$ $\sup _{z} \int_{\mathbb{R}^{d}} a\left(z, z_{1}\right) \mathrm{d} z_{1}$ in the proof of Lemma 4.1. Since it is easy to check that

$\left\{z_{1} \in C_{x y}, z_{2} \in C_{x y}, z_{3} \in C_{x y}^{\mathrm{c}}\right\} \subset\left\{\left|z_{1}-x\right| \geq K_{3}\right\} \cup\left\{\left|z_{2}-z_{1}\right| \geq K_{3}\right\} \cup\left\{\left|z_{3}-z_{2}\right| \geq K_{3}\right\}$,

we have

$$
\begin{aligned}
& \frac{1}{2 !} \int_{C_{x y}} \int_{C_{x y}} \int_{C_{x y}^{\mathrm{c}}} a\left(x, z_{1}\right) a\left(z_{1}, z_{2}\right) a\left(z_{2}, z_{3}\right) \mathrm{d} z_{3} \mathrm{~d} z_{2} \mathrm{~d} z_{1} \\
& \leq \frac{1}{2 !} \int_{\mathbb{R}^{d}} a\left(x, z_{1}\right) \int_{\mathbb{R}^{d}} a\left(z_{1}, z_{2}\right) \int_{\left|z_{3}-z_{2}\right| \geq K_{3}} a\left(z_{2}, z_{3}\right) \mathrm{d} z_{3} \mathrm{~d} z_{2} \mathrm{~d} z_{1} \\
&+\frac{1}{2 !} \int_{\mathbb{R}^{d}} a\left(x, z_{1}\right) \int_{\left|z_{2}-z_{1}\right| \geq K_{3}} a\left(z_{1}, z_{2}\right) \int_{\mathbb{R}^{d}} a\left(z_{2}, z_{3}\right) \mathrm{d} z_{3} \mathrm{~d} z_{2} \mathrm{~d} z_{1} \\
&+\frac{1}{2 !} \int_{\left|z_{1}-x\right| \geq K_{3}} a\left(x, z_{1}\right) \int_{\mathbb{R}^{d}} a\left(z_{1}, z_{2}\right) \int_{\mathbb{R}^{d}} a\left(z_{2}, z_{3}\right) \mathrm{d} z_{3} \mathrm{~d} z_{2} \mathrm{~d} z_{1} \\
& \leq \frac{1}{2 !} \int_{\mathbb{R}^{d}} a\left(x, z_{1}\right) \int_{\mathbb{R}^{d}} a\left(z_{1}, z_{2}\right) \int_{\left|z_{3}-z_{2}\right| \geq K_{3}} \frac{b}{\left|z_{3}-z_{2}\right|^{2 d+\delta}} \mathrm{d} z_{3} \mathrm{~d} z_{2} \mathrm{~d} z_{1} \\
&+\frac{1}{2 !} K \int_{\mathbb{R}^{d}} a\left(x, z_{1}\right) \int_{\left|z_{2}-z_{1}\right| \geq K_{3}} \frac{b}{\left|z_{1}-x\right|^{2 d+\delta}} \mathrm{d} z_{2} \mathrm{~d} z_{1} \\
&+\frac{1}{2 !} K^{2} \int_{\left|z_{1}-x\right| \geq K_{3}} \frac{b}{\left|z_{1}-x\right|^{2 d+\delta}} \mathrm{d} z_{1} \\
& \leq \frac{1}{2 !} \frac{w_{d} b}{(d+\delta) K_{3}^{d+\delta}} \int_{\mathbb{R}^{d}} a\left(x, z_{1}\right) \int_{\mathbb{R}^{d}} a\left(z_{1}, z_{2}\right) \mathrm{d} z_{2} \mathrm{~d} z_{1} \\
&+\frac{1}{2 !} \frac{w_{d} b}{(d+\delta) K_{3}^{d+\delta}} K \int_{\mathbb{R}^{d}} a\left(x, z_{1}\right) \mathrm{d} z_{1}+\frac{1}{2 !} \frac{w_{d} b}{(d+\delta) K_{3}^{d+\delta}} K^{2} \\
& \leq \frac{1}{2 !} \frac{3 w_{d} b K^{2}}{(d+\delta) K_{3}^{d+\delta}},
\end{aligned}
$$

where $w_{d}$ is the surface area of the unit ball in $\mathbb{R}^{d}$. Similarly, the $n$th term is less than

$$
\frac{1}{(n-1) !} \frac{n w_{d} b K^{n-1}}{(d+\delta) K_{n}^{d+\delta}}=\frac{1}{(n-1) !} \frac{2^{d+\delta} n^{d+\delta+1} w_{d} b K^{n-1}}{(d+\delta)|x-y|^{d+\delta}} .
$$


Therefore, we have

$$
\mathrm{E}\left[\left|\lambda\left(x, \eta_{s}\right)-\lambda\left(x, \eta_{s}^{\prime}\right)\right|\right] \leq \sum_{n=1}^{\infty} \frac{2\left(L+\mu_{1}\right)}{(n-1) !} \frac{2^{d+\delta} n^{d+\delta+1} w_{d} b K^{n-1}}{(d+\delta)|x-y|^{d+\delta}}=\frac{b_{1}}{|x-y|^{d+\delta}},
$$

where

$$
b_{1}=\sum_{n=1}^{\infty} \frac{\left(L+\mu_{1}\right) 2^{d+1+\delta} n^{d+1+\delta} w_{d} b K^{n-1}}{(d+\delta)(n-1) !} .
$$

Similarly, we can prove the same inequality for $\mathrm{E}\left[\left|\lambda\left(y, \eta_{t}\right)-\lambda\left(y, \eta_{t}^{\prime \prime}\right)\right|\right]$ and, hence, we have

$$
\begin{aligned}
& \left|\mathrm{E}\left[\left(\lambda\left(x, \eta_{s}\right)-\mathrm{E}\left[\lambda\left(x, \eta_{s}\right)\right]\right)\left(\lambda\left(y, \eta_{t}\right)-\mathrm{E}\left[\lambda\left(y, \eta_{t}\right)\right]\right)\right]\right| \\
& \quad \leq 2\left(L+\mu_{1}\right) \mathrm{E}\left[\left|\lambda\left(x, \eta_{s}\right)-\lambda\left(x, \eta_{s}^{\prime}\right)\right|\right]+2\left(L+\mu_{1}\right) \mathrm{E}\left[\left|\lambda\left(y, \eta_{t}\right)-\lambda\left(y, \eta_{t}^{\prime \prime}\right)\right|\right] \\
& \quad \leq \frac{4\left(L+\mu_{1}\right) b_{1}}{|x-y|^{d}} .
\end{aligned}
$$

Since $\lambda$ is bounded by $L, \mathrm{E}\left[\left(\lambda\left(x, \eta_{s}\right)-\mathrm{E}\left[\lambda\left(x, \eta_{s}\right)\right]\right)\left(\lambda\left(y, \eta_{t}\right)-\mathrm{E}\left[\lambda\left(y, \eta_{t}\right)\right]\right)\right]$ is bounded by $4 L^{2}$. This completes the proof.

Lemma 4.5. Suppose that $a(x, y) \leq b /\left(1+|x-y|^{2 d+\delta}\right)$, where $b>0$ and $\delta>0$ are constants. Let $f(x)$ be a bounded and integrable measurable function in $\mathbb{R}^{d}$ with respect to the Lebesgue measure. Then, for any $T>0$,

$$
\begin{aligned}
& \sup _{\substack{l \geq 1 \\
s, t \leq T}}\left|\frac{1}{l^{d}} \int_{\mathbb{R}^{d}} \int_{\mathbb{R}^{d}} f\left(\frac{x}{l}\right) f\left(\frac{y}{l}\right) \mathrm{E}\left[\left(\lambda\left(x, \eta_{s}\right)-\mathrm{E}\left[\lambda\left(x, \eta_{s}\right)\right]\right)\left(\lambda\left(y, \eta_{t}\right)-\mathrm{E}\left[\lambda\left(y, \eta_{t}\right)\right]\right)\right] \mathrm{d} x \mathrm{~d} y\right| \\
& \quad \leq w_{d} b_{1}\|f\|\left(\frac{1}{d}+\frac{1}{\delta}\right) \int_{\mathbb{R}^{d}}|f(y)| \mathrm{d} y,
\end{aligned}
$$

where $b_{1}$ is a constant which does not depend on $f, w_{d}$ is the surface area of the unit ball in $\mathbb{R}^{d}$, and $\|f\|=\sup _{x \in \mathbb{R}^{d}}|f(x)|$.

Proof. Fix $T>0$. Then, by Lemma 4.4, there exists a constant $b_{1}$ such that

$$
\left|\mathrm{E}\left[\left(\lambda\left(x, \eta_{s}\right)-\mathrm{E}\left[\lambda\left(x, \eta_{s}\right)\right]\right)\left(\lambda\left(y, \eta_{t}\right)-\mathrm{E}\left[\lambda\left(y, \eta_{t}\right)\right]\right)\right]\right| \leq \frac{b_{1}}{|x-y|^{d+\delta} \vee 1} .
$$

Then we have,

$$
\begin{aligned}
\left|\frac{1}{l^{d}} \int_{\mathbb{R}^{d}} \int_{\mathbb{R}^{d}} f\left(\frac{x}{l}\right) f\left(\frac{y}{l}\right) \mathrm{E}\left[\left(\lambda\left(x, \eta_{s}\right)-\mathrm{E}\left[\lambda\left(x, \eta_{s}\right)\right]\right)\left(\lambda\left(y, \eta_{t}\right)-\mathrm{E}\left[\lambda\left(y, \eta_{t}\right)\right]\right)\right] \mathrm{d} x \mathrm{~d} y\right| \\
\leq \frac{1}{l^{d}} \int_{\mathbb{R}^{d}} \int_{\mathbb{R}^{d}} \frac{b_{1}|f(x / l)||f(y / l)|}{|x-y|^{d+\delta} \vee 1} \mathrm{~d} x \mathrm{~d} y \\
=\frac{b_{1}}{l^{d}} \iint_{|x-y| \leq 1}\left|f\left(\frac{x}{l}\right)\right|\left|f\left(\frac{y}{l}\right)\right| \mathrm{d} x \mathrm{~d} y+\frac{b_{1}}{l^{d}} \iint_{|x-y|>1} \frac{|f(x / l)||f(y / l)|}{|x-y|^{d+\delta}} \mathrm{d} x \mathrm{~d} y \\
\leq \frac{b_{1}}{l^{d}} \iint_{|x-y| \leq 1}\|f\|\left|f\left(\frac{y}{l}\right)\right| \mathrm{d} x \mathrm{~d} y \\
\quad+b_{1} l^{d} \iint_{|x-y|>1} \frac{|f(x / l)||f(y / l)|}{|x-y|^{d+\delta}} \mathrm{d}\left(\frac{x}{l}\right) \mathrm{d}\left(\frac{y}{l}\right)
\end{aligned}
$$




$$
\begin{aligned}
& =\frac{w_{d} b_{1}}{d}\|f\| \int_{\mathbb{R}^{d}}|f(y)| \mathrm{d} y+b_{1} l^{d} \iint_{|x-y|>1 / l} \frac{|f(x)||f(y)|}{l^{d+\delta}|x-y|^{d+\delta}} \mathrm{d} x \mathrm{~d} y \\
& =\frac{w_{d} b_{1}}{d}\|f\| \int_{\mathbb{R}^{d}}|f(y)| \mathrm{d} y+b_{1} l^{-\delta} \int_{\mathbb{R}^{d}}|f(x)| \int_{|x-y|>1 / l} \frac{\|f\|}{|x-y|^{d+\delta}} \mathrm{d} y \mathrm{~d} x \\
& =\frac{w_{d} b_{1}}{d}\|f\| \int_{\mathbb{R}^{d}}|f(y)| \mathrm{d} y+b_{1} l^{-\delta} \int_{\mathbb{R}^{d}}|f(x)| \int_{r>1 / l} \frac{\|f\| w_{d}}{r^{d+\delta}} r^{d-1} \mathrm{~d} r \mathrm{~d} x \\
& =\frac{w_{d} b_{1}}{d}\|f\| \int_{\mathbb{R}^{d}}|f(y)| \mathrm{d} y+b_{1} l^{-\delta} \int_{\mathbb{R}^{d}}|f(x)| \frac{\|f\| w_{d}}{\delta l^{-\delta}} \mathrm{d} x \\
& =w_{d} b_{1}\|f\|\left(\frac{1}{d}+\frac{1}{\delta}\right) \int_{\mathbb{R}^{d}}|f(y)| \mathrm{d} y .
\end{aligned}
$$

Lemma 4.6. Fix $n$. For $k=1, \ldots, n$, let $\left\{Z^{(k, m)}: m=1,2, \ldots\right\}$ be a sequence of stochastic processes whose paths are elements in $D_{\mathbb{R}}[0, \infty)$ endowed with the Skorokhod topology. If, for each $1 \leq k \leq n,\left\{Z^{(k, m)}\right\}$ is relatively compact in $D_{\mathbb{R}}[0, \infty)$ and all its limits have continuous paths a.s., then the sequence $Z^{(m)}=\left(Z^{(1, m)}, \ldots, Z^{(n, m)}\right)$ is relatively compact in $D_{\mathbb{R}^{n}}[0, \infty)$ and, hence, $Z^{(1, m)}+\cdots+Z^{(n, m)}$ is relatively compact in $D_{\mathbb{R}}[0, \infty)$.

Proof. For every $\delta>0, T>0$, and $x \in D_{\mathbb{R}}[0, \infty)$ or $D_{\mathbb{R}^{n}}[0, \infty)$, we define

$$
\begin{aligned}
\omega^{\prime}(\delta, x, T)=\inf \left\{\max _{1 \leq i \leq r} \sup _{t_{i-1} \leq s \leq t<t_{i}}\left|x_{t}-x_{s}\right|: 0=t_{0}<\cdots<t_{r-1}\right. & <T \leq t_{r}, \\
& \left.\min _{1 \leq i \leq r}\left(t_{i}-t_{i-1}\right)>\delta\right\} .
\end{aligned}
$$

Let $X^{(m)}$ be $Z^{(m)}$ or $Z^{(k, m)}$ for some $k$. Then $X^{(m)}$ is relatively compact if and only if

$$
\lim _{M_{3} \rightarrow \infty} \sup _{m} \mathrm{P}\left(\left|X_{t}^{(m)}\right|>M_{3}\right)=0 \text { and } \lim _{\delta \rightarrow 0} \sup _{m} \mathrm{P}\left(\omega^{\prime}\left(\delta, X^{(m)}, T\right)>\varepsilon\right)=0
$$

for any $t \geq 0, T>0$, and $\varepsilon>0$. The first condition can be checked easily for $Z^{(m)}$. Now we check the second condition for $Z^{(m)}$. We have

$$
\begin{aligned}
\omega^{\prime}\left(\delta, Z^{(m)}, T\right) & \leq 2 \sup _{\substack{0 \leq s, t \leq(T+1) \\
|t-s| \leq \delta}}\left|Z_{t}^{(m)}-Z_{s}^{(m)}\right| \\
& \leq \sum_{k=1}^{n} 2 \sup _{\substack{0 \leq s, t \leq(T+1) \\
|t-s| \leq \delta}}\left|Z_{t}^{(k, m)}-Z_{s}^{(k, m)}\right| \\
& \leq \sum_{k=1}^{n} 2\left(2 \omega^{\prime}\left(\delta, Z^{(k, m)}, T+1\right)+\sup _{0 \leq t \leq(T+1)}\left|\Delta Z_{t}^{(k, m)}\right|\right) \\
& \leq \sum_{k=1}^{n}\left(4 \omega^{\prime}\left(\delta, Z^{(k, m)}, T+1\right)+2 \sup _{0 \leq t \leq T+1}\left|\Delta Z_{t}^{(k, m)}\right|\right),
\end{aligned}
$$

and $\sup _{0 \leq t \leq T+1}\left|\Delta x_{t}\right|$ is a measurable function in $D_{\mathbb{R}}[0, \infty)$ and continuous at any $x \in$ $C_{\mathbb{R}}[0, \infty)$. Because, for each $k$, all the limits of $\left\{Z^{(k, m)}\right\}$ have continuous paths a.s.,

$$
\sup _{0 \leq t \leq T+1}\left|\Delta Z_{t}^{(k, m)}\right|
$$


converges to 0 in distribution and, hence, in probability. Therefore, we have

$$
\begin{aligned}
& \lim _{n} \sup _{m} \mathrm{P}\left(\omega^{\prime}\left(\delta, Z^{(m)}, L\right)>\varepsilon\right) \leq \sum_{k=1}^{n}\left(\limsup _{n} \mathrm{P}\left(\omega^{\prime}\left(\delta, Z^{(k, m)}, L\right)>\frac{\varepsilon}{8 n}\right)\right. \\
&\left.\quad+\limsup _{n} \sup _{m}\left(\sup _{0 \leq t \leq L}\left|\Delta Z_{t}^{(k, m)}\right|>\frac{\varepsilon}{4 n}\right)\right) \\
&=0 .
\end{aligned}
$$

Therefore, $Z^{(m)}$ is relatively compact.

Recall that we defined $Y^{(l)}, l \geq 1$, as

$$
Y^{(l)}(f, t)=\frac{1}{l^{d / 2}}\left(\int_{\mathbb{R}^{d}} f\left(\frac{x}{l}\right) \eta_{t}(\mathrm{~d} x)-\mathrm{E}\left[\int_{\mathbb{R}^{d}} f\left(\frac{x}{l}\right) \eta_{t}(\mathrm{~d} x)\right]\right)
$$

for $t \geq 0$ and $f \in L^{1}\left(\mathbb{R}^{d}\right) \cap L^{\infty}\left(\mathbb{R}^{d}\right)$.

Let

$$
\begin{aligned}
\tilde{U}_{t}^{(l)}= & \frac{1}{l^{d / 2}} \int_{0}^{t} \int_{\mathbb{R}^{d} \times[0,+\infty)^{2}} f\left(\frac{x}{l}\right) \mathbf{1}_{\left[0, \lambda\left(x, \eta_{s-}\right)\right]}(u) N(\mathrm{~d} x, \mathrm{~d} s, \mathrm{~d} r, \mathrm{~d} u), \\
\tilde{V}_{t}^{(l)}= & \frac{1}{l^{d / 2}} \int_{0}^{t} \int_{\mathbb{R}^{d} \times[0,+\infty)^{2}} f\left(\frac{x}{l}\right) \mathbf{1}_{\left[0, \lambda\left(x, \eta_{s-}\right)\right]}(u) \mathbf{1}_{[0, t-s]}(r) N(\mathrm{~d} x, \mathrm{~d} s, \mathrm{~d} r, \mathrm{~d} u) \\
= & \frac{1}{l^{d / 2}} \int_{\{(x, s, r, u): r \geq 0, s \geq 0, s+r \leq t\}} f\left(\frac{x}{l}\right) \mathbf{1}_{\left[0, \lambda\left(x, \eta_{s-}\right)\right]}(u) N(\mathrm{~d} x, \mathrm{~d} s, \mathrm{~d} r, \mathrm{~d} u), \\
H_{t}^{(l)}= & \frac{1}{l^{d / 2}}\left(\int_{\mathbb{R}^{d} \times[0, \infty)} f\left(\frac{x}{l}\right) \mathbf{1}_{(t, \infty)}(r) \hat{\eta}_{0}(\mathrm{~d} x, \mathrm{~d} r)\right. \\
& \left.\quad-\mathrm{E}\left[\int_{\mathbb{R}^{d} \times[0, \infty)} f\left(\frac{x}{l}\right) \mathbf{1}_{(t, \infty)}(r) \hat{\eta}_{0}(\mathrm{~d} x, \mathrm{~d} r)\right]\right),
\end{aligned}
$$

and

$$
U_{t}^{(l)}=\tilde{U}_{t}^{(l)}-\mathrm{E}\left[\tilde{U}_{t}^{(l)}\right], \quad V_{t}^{(l)}=\tilde{V}_{t}^{(l)}-\mathrm{E}\left[\tilde{V}_{t}^{(l)}\right] .
$$

Note that $\int_{0}^{t} \int_{A \times[0,+\infty)^{2}} \mathbf{1}_{\left[0, \lambda\left(x, \eta_{s-}\right)\right]}(u) N(\mathrm{~d} x, \mathrm{~d} s, \mathrm{~d} r, \mathrm{~d} u)$ denotes the total number of points which are born before and at time $t$ in $A$ : it is actually a spatial pure-birth process. We denote by

$$
\int_{0}^{t} \int_{A \times[0,+\infty)^{2}} \mathbf{1}_{\left[0, \lambda\left(x, \eta_{s-}\right)\right]}(u) \mathbf{1}_{[0, t-s]}(r) N(\mathrm{~d} x, \mathrm{~d} s, \mathrm{~d} r, \mathrm{~d} u)
$$

the total number of points which are not only born but also die before time $t$ in $A$. We have

$$
Y^{(l)}(f, t)=H_{t}^{(l)}+U_{t}^{(l)}-V_{t}^{(l)} .
$$

Lemma 4.7. Suppose that $\lambda$ is bounded by $L$ and that $a(x, y) \leq b /\left(1+|x-y|^{2 d+\delta}\right)$, where $b>0$ and $\varrho>0$ are constants. Then, for each $T>0$, there exist two families $\left\{\gamma_{l}^{(i)}(\varrho): 0<\right.$ $\varrho<1, l \geq 1\}, i=1,2$, of nonnegative random variables satisfying

$$
\begin{aligned}
& \mathrm{E}\left[\left(H_{t+h}^{(l)}-H_{t}^{(l)}\right)^{2} \mid \mathcal{F}_{t}\right] \leq \mathrm{E}\left[\gamma_{l}^{(1)}(\varrho) \mid \mathcal{F}_{t}\right], \\
& \mathrm{E}\left[\left(U_{t+h}^{(l)}-U_{t}^{(l)}\right)^{2} \mid \mathcal{F}_{t}\right] \leq \mathrm{E}\left[\gamma_{l}^{(2)}(\varrho) \mid \mathcal{F}_{t}\right],
\end{aligned}
$$


for all $0 \leq t \leq T$ and $0 \leq h \leq \varrho$; in addition,

$$
\lim _{\varrho \rightarrow 0} \sup _{l} \mathrm{E}\left[\gamma_{l}^{(i)}(\varrho)\right]=0
$$

Proof. First, we estimate $\left(H_{t+h}^{(l)}-H_{t}^{(l)}\right)^{2}$. By Lemma 4.3, $\mathrm{e}^{t} H_{t}^{(l)}$ is an $\left(\mathcal{F}_{t}\right)$-martingale and its quadratic variation is

$$
\frac{1}{l^{d}} \int_{\mathbb{R}^{d} \times[0, \infty)} \mathrm{e}^{2 r} f^{2}\left(\frac{x}{l}\right) \mathbf{1}_{[0, t]}(r) \hat{\eta}_{0}(\mathrm{~d} x, \mathrm{~d} r) .
$$

Therefore,

$$
\begin{aligned}
& \mathrm{E}\left[\left(H_{t+h}^{(l)}-H_{t}^{(l)}\right)^{2} \mid \mathcal{F}_{t}\right] \\
&=\mathrm{e}^{-2(t+h)} \mathrm{E}\left[\left(\mathrm{e}^{t+h} H_{t+h}^{(l)}-\mathrm{e}^{t+h} H_{t}^{(l)}\right)^{2} \mid \mathcal{F}_{t}\right] \\
&=\mathrm{e}^{-2(t+h)} \mathrm{E}\left[\left(\mathrm{e}^{t+h} H_{t+h}^{(l)}-\mathrm{e}^{t} H_{t}^{(l)}+\mathrm{e}^{t} H_{t}^{(l)}-\mathrm{e}^{t+h} H_{t}^{(l)}\right)^{2} \mid \mathcal{F}_{t}\right] \\
& \leq 2 \mathrm{e}^{-2(t+h)} \mathrm{E}\left[\left(\mathrm{e}^{t+h} H_{t+h}^{(l)}-\mathrm{e}^{t} H_{t}^{(l)}\right)^{2} \mid \mathcal{F}_{t}\right]+2 \mathrm{e}^{-2(t+h)} \mathrm{E}\left[\left(\mathrm{e}^{t} H_{t}^{(l)}-\mathrm{e}^{t+h} H_{t}^{(l)}\right)^{2} \mid \mathcal{F}_{t}\right] \\
&= \frac{2 \mathrm{e}^{-2(t+h)}}{l^{d}} \mathrm{E}\left[\int_{\mathbb{R}^{d} \times[0, \infty)} \mathrm{e}^{2 r} f^{2}\left(\frac{x}{l}\right) \mathbf{1}_{(t, t+h]}(r) \hat{\eta}_{0}(\mathrm{~d} x, \mathrm{~d} r) \mid \mathcal{F}_{t}\right] \\
&+2\left(1-\mathrm{e}^{-h}\right)^{2}\left(H_{t}^{(l)}\right)^{2} \\
& \leq \frac{2}{l^{d}} \mathrm{E}\left[\int_{\mathbb{R}^{d} \times[0, \infty)} f^{2}\left(\frac{x}{l}\right) \mathbf{1}_{(t, t+h]}(r) \hat{\eta}_{0}(\mathrm{~d} x, \mathrm{~d} r) \mid \mathcal{F}_{t}\right]+2\left(1-\mathrm{e}^{-h}\right)^{2} \mathrm{e}^{2 t}\left(H_{t}^{(l)}\right)^{2} \\
& \leq \frac{2\left(1-\mathrm{e}^{-h}\right)}{l^{d}} \int_{\mathbb{R}^{d}} f^{2}\left(\frac{x}{l}\right) \eta_{0}(\mathrm{~d} x)+2\left(1-\mathrm{e}^{-h}\right)^{2} \mathrm{e}^{2 t}\left(H_{t}^{(l)}\right)^{2} .
\end{aligned}
$$

Let

$$
\gamma_{l}^{(1)}(\varrho)=\frac{2\left(1-\mathrm{e}^{-\varrho}\right)}{l^{d}} \int_{\mathbb{R}^{d}} f^{2}\left(\frac{x}{l}\right) \eta_{0}(\mathrm{~d} x)+2\left(1-\mathrm{e}^{-\varrho}\right)^{2} \sup _{0 \leq t \leq T} \mathrm{e}^{2 t}\left(H_{t}^{(l)}\right)^{2} .
$$

Then we have

$$
\mathrm{E}\left[\left(H_{t+h}^{(l)}-H_{t}^{(l)}\right)^{2} \mid \mathcal{F}_{t}\right] \leq \mathrm{E}\left[\gamma_{l}^{(1)}(\varrho) \mid \mathcal{F}_{t}\right]
$$

for all $0 \leq t \leq T$ and $0 \leq h \leq \varrho$, and

$$
\begin{aligned}
\mathrm{E}\left[\gamma_{l}^{(1)}(\varrho)\right] & =2 \mu_{1}\left(1-\mathrm{e}^{-\varrho}\right) \int_{\mathbb{R}^{d}} f^{2}(x) \mathrm{d} x+2\left(1-\mathrm{e}^{-\varrho}\right)^{2} \mathrm{E}\left[\sup _{0 \leq t \leq T} \mathrm{e}^{2 t}\left(H_{t}^{(l)}\right)^{2}\right] \\
& \leq 2 \mu_{1}\left(1-\mathrm{e}^{-\varrho}\right) \int_{\mathbb{R}^{d}} f^{2}(x) \mathrm{d} x+8\left(1-\mathrm{e}^{-\varrho}\right)^{2} \mathrm{E}\left[\mathrm{e}^{2 T}\left(H_{T}^{(l)}\right)^{2}\right] \\
& \leq 2 \mu_{1}\left(1-\mathrm{e}^{-\varrho}\right) \int_{\mathbb{R}^{d}} f^{2}(x) \mathrm{d} x+8 \mu_{1} T\left(1-\mathrm{e}^{-\varrho}\right)^{2} \mathrm{e}^{2 T} \int_{\mathbb{R}^{d}} f^{2}(x) \mathrm{d} x
\end{aligned}
$$

Hence, $\lim _{\varrho \rightarrow 0} \sup _{l} \mathrm{E}\left[\gamma_{l}^{(1)}(\varrho)\right]=0$.

Second, we estimate $\left(U_{t+h}^{(l)}-U_{t}^{(l)}\right)^{2}$. For each $l$, we define a square-integrable martingale $Z_{t}^{(l)}=\frac{1}{l^{d / 2}} \int_{0}^{t} \int_{\mathbb{R}^{d} \times[0,+\infty)^{2}} f\left(\frac{x}{l}\right) \mathbf{1}_{\left[0, \lambda\left(x, \eta_{s-}\right)\right]}(u)\left(N(\mathrm{~d} x, \mathrm{~d} s, \mathrm{~d} r, \mathrm{~d} u)-\mathrm{d} x \mathrm{e}^{-r} \mathrm{~d} r \mathrm{~d} u \mathrm{~d} s\right)$ 
and a continuous process

$$
A_{t}^{(l)}=\frac{1}{l^{d / 2}} \int_{0}^{t} \int_{\mathbb{R}^{d}} f\left(\frac{x}{l}\right)\left(\lambda\left(x, \eta_{s-}\right)-\mathrm{E}\left[\lambda\left(x, \eta_{s-}\right)\right]\right) \mathrm{d} x \mathrm{~d} s .
$$

Then

$$
U_{t+h}^{(l)}-U_{t}^{(l)}=\left(Z_{t+h}^{(l)}-Z_{t}^{(l)}\right)+\left(A_{t+h}^{(l)}-A_{t}^{(l)}\right) .
$$

Since the quadratic variation of $Z_{t}^{(l)}$ is

$$
\left[Z^{(l)}\right]_{t}=\frac{1}{l^{d}} \int_{0}^{t} \int_{\mathbb{R}^{d} \times[0,+\infty)^{2}} f\left(\frac{x}{l}\right)^{2} \mathbf{1}_{\left[0, \lambda\left(x, \eta_{s-}\right)\right]}(u) N(\mathrm{~d} x, \mathrm{~d} s, \mathrm{~d} r, \mathrm{~d} u),
$$

we have

$$
\begin{aligned}
\mathrm{E}\left[\left(Z_{t+h}^{(l)}-Z_{t}^{(l)}\right)^{2} \mid \mathcal{F}_{t}\right] & =\mathrm{E}\left[\left(Z_{t+h}^{(l)}\right)^{2}-\left(Z_{t}^{(l)}\right)^{2} \mid \mathcal{F}_{t}\right] \\
& =\mathrm{E}\left[\left[Z^{(l)}\right]_{t+h}-\left[Z^{(l)}\right]_{t} \mid \mathcal{F}_{t}\right] \\
& =\frac{1}{l^{d}} \mathrm{E}\left[\int_{t}^{t+h} \int_{\mathbb{R}^{d}} f\left(\frac{x}{l}\right)^{2} \lambda\left(x, \eta_{s-}\right) \mathrm{d} x \mathrm{~d} s \mid \mathcal{F}_{t}\right] \\
& \leq h L \int f^{2}(x) \mathrm{d} x
\end{aligned}
$$

By Lemma 4.5, let

$$
\begin{aligned}
& \sup _{\substack{l \geq 1 \\
s, t \leq T+1}} \mid \frac{1}{l^{d}} \int_{\mathbb{R}^{d}} \int_{\mathbb{R}^{d}} f\left(\frac{x}{l}\right) f\left(\frac{y}{l}\right) \\
& \quad \times \mathrm{E}\left[\left(\lambda\left(x, \eta_{s}\right)-\mathrm{E}\left[\lambda\left(x, \eta_{s}\right)\right]\right)\left(\lambda\left(y, \eta_{t}\right)-\mathrm{E}\left[\lambda\left(y, \eta_{t}\right)\right]\right)\right] \mathrm{d} x \mathrm{~d} y \mid \\
& \quad=K_{5} \\
& \quad<\infty
\end{aligned}
$$

and, hence, we have

$$
\begin{aligned}
\mathrm{E}\left[\left(A_{t+h}^{(l)}-A_{t}^{(l)}\right)^{2}\right]= & \mathrm{E}\left[\left(\frac{1}{l^{d / 2}} \int_{t}^{t+h} \int_{\mathbb{R}^{d}} f\left(\frac{x}{l}\right)\left(\lambda\left(x, \eta_{s-}\right)-\mathrm{E}\left[\lambda\left(x, \eta_{s-}\right)\right]\right) \mathrm{d} x \mathrm{~d} s\right)^{2}\right] \\
= & \frac{1}{l^{d}} \int_{t}^{t+h} \int_{t}^{t+h} \int_{\mathbb{R}^{d}} \int_{\mathbb{R}^{d}} f\left(\frac{x}{l}\right) f\left(\frac{y}{l}\right) \\
& \times \mathrm{E}\left[\left(\lambda\left(x, \eta_{r}\right)-\mathrm{E}\left[\lambda\left(x, \eta_{r}\right)\right]\right)\left(\lambda\left(y, \eta_{s}\right)-\mathrm{E}\left[\lambda\left(y, \eta_{s}\right)\right]\right)\right] \mathrm{d} x \mathrm{~d} y \mathrm{~d} s \mathrm{~d} r \\
\leq & K_{5} h^{2},
\end{aligned}
$$

and the expectation of the square of the variation of $A^{(l)}$ is

$$
\left(\mathrm{E}\left[\left(\int_{0}^{t}\left|\mathrm{~d} A_{t}^{(l)}\right|\right)^{2}\right]\right)^{1 / 2} \leq \lim _{n \rightarrow \infty} \sum_{k=1}^{2^{n}}\left(\mathrm{E}\left[\left(A_{k t / 2^{n}}^{(l)}-A_{(k-1) t / 2^{n}}^{(l)}\right)^{2}\right]\right)^{1 / 2} \leq \sqrt{K_{5}} t .
$$

By the Kolmogorov criterion (see (2.1) Theorem, Chapter I of Revuz and Yor (2004)),

$$
\mathrm{E}\left[\left(\sup _{\substack{s, t \leq T+1 \\ s \neq t}} \frac{\left|A_{t}^{(l)}-A_{s}^{(l)}\right|}{|t-s|^{1 / 3}}\right)^{2}\right]<\infty
$$


and the expectations can be uniformly bounded by a constant which only depends on $K_{5}$ and $T$ and does not depend on $l$. Now let

$$
\gamma_{l}^{(2)}(\varrho)=2 \varrho L \int_{\mathbb{R}^{d}} f^{2}(x) \mathrm{d} x+2 \varrho^{2 / 3}\left(\sup _{\substack{s, t \leq T+1 \\ s \neq t}} \frac{\left|A_{t}^{(l)}-A_{s}^{(l)}\right|}{|t-s|^{1 / 3}}\right)^{2} .
$$

Then

$$
\begin{aligned}
\mathrm{E}\left[\left(U_{t, h}^{(l)}-\mathrm{E}\left[U_{t, h}^{(l)}\right]\right)^{2} \mid \mathcal{F}_{t}\right] & \leq 2 \mathrm{E}\left[\left(Z_{t+h}^{(l)}-Z_{t}^{(l)}\right)^{2} \mid \mathcal{F}_{t}\right]+2 \mathrm{E}\left[\left(A_{t+h}^{(l)}-A_{t}^{(l)}\right)^{2} \mid \mathcal{F}_{t}\right] \\
& \leq \mathrm{E}\left[\gamma_{l}^{(2)}(\varrho) \mid \mathcal{F}_{t}\right]
\end{aligned}
$$

and

$$
\lim _{\varrho \rightarrow 0} \sup _{l} \mathrm{E}\left[\gamma_{l}^{(2)}(\varrho)\right]=0
$$

For any $x, y \in \mathbb{R}^{d}$, we define $q(x, y)=|x-y| \wedge 1$.

Lemma 4.8. Suppose that $\lambda$ is bounded by $L$ and that $a(x, y) \leq b /\left(1+|x-y|^{2 d+\delta}\right)$, where $b>0$ and $\delta>0$ are constants. Fix $T>0$. Then there exists $C>0$ such that

$$
\mathrm{E}\left[q^{2}\left(V_{t+h}^{(l)}, V_{t}^{(l)}\right) q^{2}\left(V_{t}^{(l)}, V_{t-h}^{(l)}\right)\right] \leq C h^{2}
$$

for all $0 \leq t \leq T+1$ and $0 \leq h \leq t$. In addition,

$$
\lim _{h \rightarrow 0} \sup _{l} \mathrm{E}\left[\left(V_{h}^{(l)}\right)^{2}\right]=0 .
$$

Proof. Fix $t, h$, and $l$. For $0 \leq t^{\prime} \leq t+h$, let

$$
\begin{aligned}
& X_{t^{\prime}}=\frac{1}{l^{d / 2}} \int_{\left\{(x, s, r, u) \mid t \leq r+s \leq t+h, r \geq 0,0 \leq s \leq t^{\prime}\right\}} f\left(\frac{x}{l}\right) \\
& \times \mathbf{1}_{\left[0, \lambda\left(x, \eta_{s-}\right)\right]}(u)\left(N(\mathrm{~d} x, \mathrm{~d} s, \mathrm{~d} r, \mathrm{~d} u)-\mathrm{d} x \mathrm{~d} s \mathrm{e}^{-r} \mathrm{~d} r \mathrm{~d} u\right), \\
& Y_{t^{\prime}}=\frac{1}{l^{d / 2}} \int_{\left\{(x, s, r, u) \mid t-h \leq r+s \leq t, r \geq 0,0 \leq s \leq t^{\prime}\right\}} f\left(\frac{x}{l}\right) \\
& \times \mathbf{1}_{\left[0, \lambda\left(x, \eta_{s-}\right)\right]}(u)\left(N(\mathrm{~d} x, \mathrm{~d} s, \mathrm{~d} r, \mathrm{~d} u)-\mathrm{d} x \mathrm{~d} s \mathrm{e}^{-r} \mathrm{~d} r \mathrm{~d} u\right) .
\end{aligned}
$$

Then $\left\{X_{t^{\prime}}, Y_{t^{\prime}}: 0 \leq t^{\prime} \leq t+h\right\}$ are two martingales. Let

$$
\begin{aligned}
B_{+} & =\frac{1}{l^{d / 2}} \int_{0}^{t+h} \int_{\mathbb{R}^{d}} f\left(\frac{x}{l}\right)\left(\lambda\left(x, \eta_{s-}\right)-\mathrm{E}\left[\lambda\left(x, \eta_{s-}\right)\right]\right)\left(\mathrm{e}^{-(t-s)} \wedge 1-\mathrm{e}^{-(t+h-s)}\right) \mathrm{d} x \mathrm{~d} s \\
& =\int_{0}^{t+h}\left(\mathrm{e}^{-(t-s)} \wedge 1-\mathrm{e}^{-(t+h-s)}\right) \mathrm{d} A_{s}^{(l)}, \\
B_{-} & =\frac{1}{l^{d / 2}} \int_{0}^{t} \int_{\mathbb{R}^{d}} f\left(\frac{x}{l}\right)\left(\lambda\left(x, \eta_{s-}\right)-\mathrm{E}\left[\lambda\left(x, \eta_{s-}\right)\right]\right)\left(\mathrm{e}^{-(t-h-s)} \wedge 1-\mathrm{e}^{-(t-s)}\right) \mathrm{d} x \mathrm{~d} s \\
& =\int_{0}^{t}\left(\mathrm{e}^{-(t-h-s)} \wedge 1-\mathrm{e}^{-(t-s)}\right) \mathrm{d} A_{s}^{(l)} .
\end{aligned}
$$


Then we have

$$
V_{t+h}^{(l)}-V_{t}^{(l)}=X_{t+h}+B_{+} \quad \text { and } \quad V_{t}^{(l)}-V_{t-h}^{(l)}=Y_{t+h}+B_{-}=Y_{t}+B_{-} .
$$

Therefore,

$$
\begin{aligned}
q^{2}\left(V_{t+h}^{(l)}, V_{t}^{(l)}\right) q^{2}\left(V_{t}^{(l)}, V_{t-h}^{(l)}\right) & =\left(\left(X_{t+h}+B_{+}\right)^{2} \wedge 1\right)\left(\left(Y_{t+h}+B_{-}\right)^{2} \wedge 1\right) \\
& \leq 4\left(X_{t+h}^{2} \wedge 1+B_{+}^{2} \wedge 1\right)\left(Y_{t+h}^{2} \wedge 1+B_{-}^{2} \wedge 1\right) \\
& \leq 4\left(X_{t+h}^{2} \wedge 1\right)\left(Y_{t+h}^{2} \wedge 1\right)+8 B_{+}^{2} \wedge 1+8 B_{-}^{2} \wedge 1
\end{aligned}
$$

By (4.6) we have

$$
\begin{aligned}
\mathrm{E}\left[B_{+}^{2}\right] & =\mathrm{E}\left[\int_{0}^{t+h}\left(\mathrm{e}^{-(t-s)} \wedge 1-\mathrm{e}^{-(t+h-s)}\right) \mathrm{d} A_{s}^{(l)}\right]^{2} \\
& \leq\left(1-\mathrm{e}^{-h}\right)^{2} \mathrm{E}\left[\left(\int_{0}^{t+h}\left|\mathrm{~d} A_{t}^{(l)}\right|\right)^{2}\right] \\
& \leq K_{5}(t+h)^{2} h^{2} \\
& \leq K_{5}(2 T+2)^{2} h^{2}
\end{aligned}
$$

Similarly, $\mathrm{E}\left[B_{-}^{2}\right] \leq K_{5}(2 T+2)^{2} h^{2}$.

We define two stopping times

$$
S_{1}=\inf \left\{t^{\prime}:\left|X_{t^{\prime}}\right| \geq 1\right\} \wedge(t+h) \quad \text { and } \quad S_{2}=\inf \left\{t^{\prime}:\left|Y_{t^{\prime}}\right| \geq 1\right\} \wedge(t+h) .
$$

Because $X_{0}=Y_{0}=0$ and the jumps of $X_{t^{\prime}}$ and $Y_{t^{\prime}}$ are bounded by $\|f\| / l^{d / 2}$, the stopped processes $X_{S_{1} \wedge t^{\prime}}$ and $Y_{S_{2} \wedge t^{\prime}}$ are bounded martingales and

$$
\left(X_{t+h}^{2} \wedge 1\right)\left(Y_{t+h}^{2} \wedge 1\right) \leq X_{S_{1} \wedge(t+h)}^{2} Y_{S_{2} \wedge(t+h)}^{2} .
$$

By Itô's formula,

$$
\begin{aligned}
X_{S_{1} \wedge(t+h)}^{2} Y_{S_{2} \wedge(t+h)}^{2} & \int_{0}^{S_{1} \wedge(t+h)} 2 X_{S_{1} \wedge t^{\prime}-} Y_{S_{2} \wedge t^{\prime}-}^{2} \mathrm{~d} X_{t^{\prime}}+\int_{0}^{S_{2} \wedge(t+h)} 2 X_{S_{1} \wedge t^{\prime}-}^{2} Y_{S_{2} \wedge t^{\prime}-} \mathrm{d} Y_{t^{\prime}} \\
& +\sum_{t^{\prime} \leq(t+h) \wedge\left(S_{1} \vee S_{2}\right)}\left(X_{t^{\prime}}^{2} Y_{t^{\prime}}^{2}-X_{t^{\prime}-}^{2} Y_{t^{\prime}-}^{2}-2 X_{t^{\prime}-} Y_{t^{\prime}-}^{2} \Delta X_{t^{\prime}}-2 X_{t^{\prime}-}^{2} Y_{t^{\prime}-} \Delta Y_{t^{\prime}}\right) .
\end{aligned}
$$

We have

$$
\begin{aligned}
X_{t^{\prime}}^{2} Y_{t^{\prime}}^{2}- & X_{t^{\prime}-}^{2} Y_{t^{\prime}-}^{2}-2 X_{t^{\prime}-} Y_{t^{\prime}-}^{2} \Delta X_{t^{\prime}}-2 X_{t^{\prime}-}^{2} Y_{t^{\prime}-} \Delta Y_{t^{\prime}} \\
= & \left(X_{t^{\prime}-}+\Delta X_{t^{\prime}}\right)^{2}\left(Y_{t^{\prime}-}+\Delta Y_{t^{\prime}}\right)^{2}-X_{t^{\prime}-}^{2} Y_{t^{\prime}-}^{2}-2 X_{t^{\prime}-} Y_{t^{\prime}-}^{2} \Delta X_{t^{\prime}}-2 X_{t^{\prime}-}^{2} Y_{t^{\prime}-} \Delta Y_{t^{\prime}} \\
= & \left(\Delta X_{t^{\prime}}\right)^{2} Y_{t^{\prime}-}^{2}+\left(\Delta Y_{t^{\prime}}\right)^{2} X_{t^{\prime}-}^{2}+\left(\Delta X_{t^{\prime}}\right)^{2}\left(\Delta Y_{t^{\prime}}\right)^{2} \\
& +2 X_{t^{\prime}-}\left(\Delta Y_{t^{\prime}-}\right)^{2} \Delta X_{t^{\prime}}+2 Y_{t^{\prime}-}\left(\Delta X_{t^{\prime}-}\right)^{2} \Delta Y_{t^{\prime}}+4 \Delta X_{t^{\prime}} \Delta Y_{t^{\prime}} X_{t^{\prime}-} Y_{t^{\prime}-} \\
= & \left(\Delta X_{t^{\prime}}\right)^{2} Y_{t^{\prime}-}^{2}+\left(\Delta Y_{t^{\prime}}\right)^{2} X_{t^{\prime}-}^{2},
\end{aligned}
$$

where the last equality is true because $X_{t^{\prime}}$ and $Y_{t^{\prime}}$ have no common jumps. Since

$$
\int_{0}^{S_{1} \wedge \cdot} 2 X_{S_{1} \wedge t^{\prime}-} Y_{S_{2} \wedge t^{\prime}-}^{2} \mathrm{~d} X_{t^{\prime}} \text { and } \int_{0}^{S_{1} \wedge \cdot} 2 X_{S_{1} \wedge t^{\prime}-}^{2} Y_{S_{2} \wedge t^{\prime}-} \mathrm{d} Y_{t^{\prime}}
$$


are martingales,

$$
\begin{aligned}
\mathrm{E}\left[X_{S_{1} \wedge(t+h)}^{2} Y_{S_{2} \wedge(t+h)}^{2}\right] & \\
= & \mathrm{E}\left[\int_{0}^{S_{1} \wedge(t+h)} Y_{S_{2} \wedge\left(t^{\prime}\right)-}^{2} \mathrm{~d}[X]_{t^{\prime}}\right]+\mathrm{E}\left[\int_{0}^{S_{2} \wedge(t+h)} X_{S_{1} \wedge\left(t^{\prime}\right)-}^{2} \mathrm{~d}[Y]_{t^{\prime}}\right] \\
= & \frac{1}{l^{d}} \mathrm{E}\left[\int_{0}^{S_{1} \wedge(t+h)} \int_{\mathbb{R}^{d} \times[0,+\infty)^{2}} Y_{S_{2} \wedge s-}^{2} f^{2}\left(\frac{x}{l}\right) \mathbf{1}_{\left[0, \lambda\left(x, \eta_{s-}\right)\right]}(u)\right. \\
& \left.\times \mathbf{1}_{(t-s, t+h-s]}(r) N(\mathrm{~d} x, \mathrm{~d} s, \mathrm{~d} r, \mathrm{~d} u)\right] \\
& +\frac{1}{l^{d}} \mathrm{E}\left[\int_{0}^{S_{2} \wedge(t+h)} \int_{\mathbb{R}^{d} \times[0,+\infty)^{2}} X_{S_{1} \wedge s-}^{2} f^{2}\left(\frac{x}{l}\right) \mathbf{1}_{\left[0, \lambda\left(x, \eta_{s-}\right)\right]}(u)\right. \\
\leq & L\left(1-\mathrm{e}^{-h}\right) \int_{\mathbb{R}^{d}} f^{2}(x) \mathrm{d} x \int_{0}^{t+h} \mathrm{E}\left[Y_{S_{2} \wedge s}^{2}\right] \mathrm{d} s \\
& +L\left(1-\mathrm{e}^{-h}\right) \int_{\mathbb{R}^{d}} f^{2}(x) \mathrm{d} x \int_{0}^{t+h} \mathrm{E}\left[X_{S_{1} \wedge s}^{2}\right] \mathrm{d} s .
\end{aligned}
$$

Because

$$
\begin{aligned}
\mathrm{E}\left[Y_{S_{2} \wedge s}^{2}\right] \leq & \mathrm{E}\left[Y_{t+h}^{2}\right] \\
\leq & \frac{1}{l^{d}} \mathrm{E}\left[\int_{0}^{t+h} \int_{\mathbb{R}^{d} \times[0,+\infty)^{2}} f^{2}\left(\frac{x}{l}\right)\right. \\
& \left.\quad \times \mathbf{1}_{\left[0, \lambda\left(x, \eta_{s-}\right)\right]}(u) \mathbf{1}_{(t-h-s, t-s]}(r) N(\mathrm{~d} x, \mathrm{~d} s, \mathrm{~d} r, \mathrm{~d} u)\right] \\
\leq & L\left(1-\mathrm{e}^{-h}\right) \int_{\mathbb{R}^{d}} f^{2}(x) \mathrm{d} x
\end{aligned}
$$

and, similarly,

$$
\mathrm{E}\left[X_{S_{1} \wedge s}^{2}\right] \leq \mathrm{E}\left[X_{t+h}^{2}\right] \leq L\left(1-\mathrm{e}^{-h}\right) \int_{\mathbb{R}^{d}} f^{2}(x) \mathrm{d} x,
$$

we have

$$
\begin{aligned}
& \mathrm{E}\left[X_{S_{1} \wedge(t+h)}^{2} Y_{S_{2} \wedge(t+h)}^{2}\right] \\
& \leq L\left(1-\mathrm{e}^{-h}\right) \int_{\mathbb{R}^{d}} f^{2}(x) \mathrm{d} x \int_{0}^{t+h} \mathrm{E}\left[Y_{S_{2} \wedge s}^{2}\right] \mathrm{d} s \\
&+L\left(1-\mathrm{e}^{-h}\right) \int_{\mathbb{R}^{d}} f^{2}(x) \mathrm{d} x \int_{0}^{t+h} \mathrm{E}\left[X_{S_{1} \wedge s}^{2}\right] \mathrm{d} s . \\
& \leq 2(t+h) L^{2}\left(1-\mathrm{e}^{-h}\right)^{2}\left(\int_{\mathbb{R}^{d}} f^{2}(x) \mathrm{d} x\right)^{2} \\
& \leq 2(T+1) L^{2}\left(\int_{\mathbb{R}^{d}} f^{2}(x) \mathrm{d} x\right)^{2} h^{2} .
\end{aligned}
$$

Now by (4.7) we have

$$
\mathrm{E}\left[q^{2}\left(V_{t+h}^{(l)}, V_{t}^{(l)}\right) q^{2}\left(V_{t}^{(l)}, V_{t-h}^{(l)}\right)\right] \leq C h^{2},
$$


where $C$ depends only on $T$. We can also prove that

$$
\lim _{h \rightarrow 0} \sup _{l} \mathrm{E}\left[\left(V_{h}^{(l)}\right)^{2}\right]=0 .
$$

Lemma 4.9. Suppose that $\left\{W_{l}^{(n)}: n \in \mathbb{N}, l \in \mathbb{R}, l \geq 1\right\}$ and $\left\{W_{l}: l \in \mathbb{R}, l \geq 1\right\}$ are two families of random variables such that, for each $n,\left\{W_{l}^{(n)}: l \geq 1\right\}$ converges weakly to a random variable $V^{(n)}$ with normal distribution as $l \rightarrow \infty$ and

$$
\lim _{n \rightarrow \infty} \sup _{l} \mathrm{E}\left[\left(W_{l}^{(n)}-W_{l}\right)^{2}\right]=0 .
$$

Then $\left\{W_{l}: l \in \mathbb{R}, l \geq 1\right\}$ converges weakly to a normal distribution.

Proof. First, we show that $\left\{V^{(n)}\right\}$ converges weakly. For any $t \in \mathbb{R}$ and any $m, n \in \mathbb{N}$, we have

$$
\begin{aligned}
\left|\mathrm{E}\left[\exp \left(\mathrm{i} t V^{(m)}\right)\right]-\mathrm{E}\left[\exp \left(\mathrm{i} t V^{(n)}\right)\right]\right| & =\lim _{l \rightarrow \infty}\left|\mathrm{E}\left[\exp \left(\mathrm{i} t W_{l}^{(m)}\right)\right]-\mathrm{E}\left[\exp \left(\mathrm{i} t W_{l}^{(n)}\right)\right]\right| \\
& \leq \sup _{l} \mathrm{E}\left[\left|\exp \left(\mathrm{i} t W_{l}^{(m)}\right)-\exp \left(\mathrm{i} t W_{l}^{(n)}\right)\right|\right] \\
& \leq \sup _{l} \mathrm{E}\left[\left|\exp \left(\mathrm{i} t W_{l}^{(m)}\right)\left(1-\exp \left(\mathrm{i} t\left(W_{l}^{(n)}-W_{l}^{(m)}\right)\right)\right)\right|\right] \\
& =\sup _{l} \mathrm{E}\left[\left|1-\exp \left(\mathrm{i} t\left(W_{l}^{(n)}-W_{l}^{(m)}\right)\right)\right|\right] \\
& \leq \sup _{l} t \mathrm{E}\left[\left|W_{l}^{(n)}-W_{l}^{(m)}\right|\right] \\
& \leq \sup _{l} t\left(\mathrm{E}\left[\left(W_{l}^{(n)}-W_{l}^{(m)}\right)^{2}\right]\right)^{1 / 2} \\
& \rightarrow 0 \quad \text { as } m, n \rightarrow \infty .
\end{aligned}
$$

Note that the above convergence is uniform for $t$ on any finite interval. Hence, $\left\{V^{(n)}\right\}$ converges weakly. Since all the $\left\{V^{(n)}\right\}$ have normal distributions, the limit has a normal distribution also. Let $V$ denote a random variable with the limit normal distribution. We want to show that $\left\{W_{l}: l \in \mathbb{R}, l \geq 1\right\}$ converges weakly to $V$. Fix $t \in \mathbb{R}$. For any $n$,

$$
\begin{aligned}
\left|\mathrm{E}\left[\exp \left(\mathrm{i} t W_{l}\right)\right]-\mathrm{E}\left[\mathrm{e}^{\mathrm{i} t V}\right]\right| \leq & \left|\mathrm{E}\left[\exp \left(\mathrm{i} t W_{l}\right)\right]-\mathrm{E}\left[\exp \left(\mathrm{i} t W_{l}^{(n)}\right)\right]\right| \\
& +\left|\mathrm{E}\left[\exp \left(\mathrm{i} t W_{l}^{(n)}\right)\right]-\mathrm{E}\left[\exp \left(\mathrm{i} t V^{(n)}\right)\right]\right| \\
& +\left|\mathrm{E}\left[\exp \left(\mathrm{i} t V^{(n)}\right)\right]-\mathrm{E}\left[\mathrm{e}^{\mathrm{i} t V}\right]\right| \\
\leq & \sup _{l} t\left(\mathrm{E}\left[\left(W_{l}-W_{l}^{(n)}\right)^{2}\right]\right)^{1 / 2}+\left|\mathrm{E}\left[\exp \left(\mathrm{i} t W_{l}^{(n)}\right)\right]-\mathrm{E}\left[\exp \left(\mathrm{i} t V^{(n)}\right)\right]\right| \\
& +\left|\mathrm{E}\left[\exp \left(\mathrm{i} t V^{(n)}\right)\right]-\mathrm{E}\left[\mathrm{e}^{\mathrm{i} t V}\right]\right| .
\end{aligned}
$$

Let $l \rightarrow \infty$. Then the second term in the last line disappears. We have $\limsup _{l \rightarrow \infty}\left|\mathrm{E}\left[\exp \left(\mathrm{i} t W_{l}\right)\right]-\mathrm{E}\left[\mathrm{e}^{\mathrm{i} t V}\right]\right| \leq \sup _{l} t\left(\mathrm{E}\left[\left(W_{l}-W_{l}^{(n)}\right)^{2}\right]\right)^{1 / 2}+\left|\mathrm{E}\left[\exp \left(\mathrm{i} t V^{(n)}\right)\right]-\mathrm{E}\left[\mathrm{e}^{\mathrm{i} t V}\right]\right|$.

Since the left-hand side of the above inequality does not depend on $n$, we have, letting $n \rightarrow \infty$,

$$
\limsup _{l \rightarrow \infty}\left|\mathrm{E}\left[\exp \left(\mathrm{i} t W_{l}\right)\right]-\mathrm{E}\left[\mathrm{e}^{\mathrm{i} t V}\right]\right| \rightarrow 0 .
$$

This completes the proof. 
Proof of Theorem 2.2. First we prove that $H^{(l)}, U^{(l)}$, and $V^{(l)}$ are relatively compact. Using the estimates in Lemmas 4.7 and 4.8, by Theorems 8.6 and 8.8 of Ethier and Kurtz (1985), it remains to show that, for each $t \geq 0$, the families $\left\{H_{t}^{(l)}: l \geq 1\right\},\left\{U_{t}^{(l)}: l \geq 1\right\}$, and $\left\{V_{t}^{(l)}: l \geq 1\right\}$ are relatively compact. In order to do this, we show that the finite-dimensional distributions of $Y^{(l)}(f, t)$ converge weakly to multivariate normal distributions. By Theorem 4.2, if $f(x)$ is the linear combination of indicator functions of Riemann measurable sets in $\mathbb{R}^{d}$, then the finitedimensional distributions of $Y^{(l)}(f, \cdot)$ converge weakly to multivariate normal distributions. If $f$ is a bounded integrable function, we can find a sequence $\left\{g_{n}(x): n=1, \ldots\right\}$ such that $g_{n}$ is a linear combination of indicator functions of Riemann measurable sets, and

$$
\sup _{n}\left\|g_{n}\right\|<\infty \quad \text { and } \quad \lim _{n} \int_{\mathbb{R}^{d}}\left|g_{n}(x)-f(x)\right| \mathrm{d} x=0
$$

Equation (4.8) actually implies that

$$
\lim _{n} \int_{\mathbb{R}^{d}}\left(g_{n}(x)-f(x)\right)^{2} \mathrm{~d} x=0 .
$$

Now if we can show that, for any $t \geq 0$,

$$
\begin{gathered}
\sup _{l} \mathrm{E}\left[\frac{1}{l^{d / 2}}\left(\int_{\mathbb{R}^{d}} f\left(\frac{x}{l}\right) \eta_{t}(\mathrm{~d} x)-\mathrm{E}\left[\int_{\mathbb{R}^{d}} f\left(\frac{x}{l}\right) \eta_{t}(\mathrm{~d} x)\right]\right)\right. \\
\left.\quad-\frac{1}{l^{d / 2}}\left(\int_{\mathbb{R}^{d}} g_{n}\left(\frac{x}{l}\right) \eta_{t}(\mathrm{~d} x)-\mathrm{E}\left[\int_{\mathbb{R}^{d}} g_{n}\left(\frac{x}{l}\right) \eta_{t}(\mathrm{~d} x)\right]\right)\right]^{2} \\
=\sup _{l} \mathrm{E}\left[\frac { 1 } { l ^ { d } } \left(\int_{\mathbb{R}^{d}}\left(f\left(\frac{x}{l}\right)-g_{n}\left(\frac{x}{l}\right)\right) \eta_{t}(\mathrm{~d} x)\right.\right. \\
\left.\left.\quad-\mathrm{E}\left[\int_{\mathbb{R}^{d}}\left(f\left(\frac{x}{l}\right)-g_{n}\left(\frac{x}{l}\right)\right) \eta_{t}(\mathrm{~d} x)\right]\right)^{2}\right] \\
\rightarrow 0 \quad \text { as } n \rightarrow \infty
\end{gathered}
$$

then, by Lemma 4.9, the finite-dimensional distributions of $Y^{(l)}(f, \cdot)$ converge weakly to multivariate normal distributions. We have

$$
\begin{gathered}
\mathrm{E}\left[\frac{1}{l^{d}}\left(\int_{\mathbb{R}^{d}}\left(f\left(\frac{x}{l}\right)-g_{n}\left(\frac{x}{l}\right)\right) \eta_{t}(\mathrm{~d} x)-\mathrm{E}\left[\int_{\mathbb{R}^{d}}\left(f\left(\frac{x}{l}\right)-g_{n}\left(\frac{x}{l}\right)\right) \eta_{t}(\mathrm{~d} x)\right]\right)^{2}\right] \\
\leq \frac{3}{l^{d}} \mathrm{E}\left[\left(\int_{0}^{t} \int_{\mathbb{R}^{d} \times[0,+\infty)^{2}}\left(f\left(\frac{x}{l}\right)-g_{n}\left(\frac{x}{l}\right)\right) \mathbf{1}_{\left[0, \lambda\left(x, \eta_{s-}\right)\right]}(u) \mathbf{1}_{(t-s, \infty)}(r)\right.\right. \\
\left.\left.\times\left(N(\mathrm{~d} x, \mathrm{~d} s, \mathrm{~d} r, \mathrm{~d} u)-\mathrm{d} x \mathrm{~d} s \mathrm{e}^{-r} \mathrm{~d} r \mathrm{~d} u\right)\right)^{2}\right] \\
+\frac{3}{l^{d}} \mathrm{E}\left[\left(\int_{0}^{t} \int_{\mathbb{R}^{d}}\left(f\left(\frac{x}{l}\right)-g_{n}\left(\frac{x}{l}\right)\right)\left(\lambda\left(x, \eta_{s-}\right)-\mathrm{E}\left[\lambda\left(x, \eta_{s-}\right)\right] \mathrm{e}^{-(t-s)} \mathrm{d} x \mathrm{~d} s\right)^{2}\right]\right. \\
+\frac{3}{l^{d}} \mathrm{E}\left[\left(\int_{\mathbb{R}^{d} \times[0,+\infty)}\left(f\left(\frac{x}{l}\right)-g_{n}\left(\frac{x}{l}\right)\right) \mathbf{1}_{(t, \infty)}(r) \hat{\eta}(\mathrm{d} x, \mathrm{~d} r)\right.\right. \\
\left.\left.-\mathrm{E}\left[\int_{\mathbb{R}^{d} \times[0,+\infty)}\left(f\left(\frac{x}{l}\right)-g_{n}\left(\frac{x}{l}\right)\right) \mathbf{1}_{(t, \infty)}(r) \hat{\eta}(\mathrm{d} x, \mathrm{~d} r)\right]\right)^{2}\right] .
\end{gathered}
$$


In order to compute the first term on the right-hand side of (4.9), we fix $t$ and define a squareintegrable martingale with respect to its natural filtration,

$$
\begin{aligned}
X_{t^{\prime}}^{(l)}=\frac{3}{l^{d / 2}} \int_{0}^{t^{\prime}} \int_{\mathbb{R}^{d} \times[0,+\infty)^{2}} & \left(f\left(\frac{x}{l}\right)-g_{n}\left(\frac{x}{l}\right)\right) \mathbf{1}_{\left[0, \lambda\left(x, \eta_{s-}\right)\right]}(u) \mathbf{1}_{(t-s, \infty)}(r) \\
& \times\left(N(\mathrm{~d} x, \mathrm{~d} s, \mathrm{~d} r, \mathrm{~d} u)-\mathrm{d} x \mathrm{~d} s \mathrm{e}^{-r} \mathrm{~d} r \mathrm{~d} u\right),
\end{aligned}
$$

where $0 \leq t^{\prime} \leq t$. Then the first term on the right-hand-side of (4.9) satisfies

$$
\begin{aligned}
\mathrm{E}\left[\left(X_{t}^{(l)}\right)^{2}\right] & =\frac{3}{l^{d}} \int_{0}^{t} \int_{\mathbb{R}^{d} \times[0,+\infty)^{2}}\left(f\left(\frac{x}{l}\right)-g_{n}\left(\frac{x}{l}\right)\right)^{2} \\
& \times \mathbf{1}_{\left[0, \lambda\left(x, \eta_{s-}\right)\right]}(u) \mathbf{1}_{(t-s, \infty)}(r) N(\mathrm{~d} x, \mathrm{~d} s, \mathrm{~d} r, \mathrm{~d} u) \\
& \leq \frac{3 L}{l^{d}} \int_{0}^{t} \int_{\mathbb{R}^{d}}\left(f\left(\frac{x}{l}\right)-g_{n}\left(\frac{x}{l}\right)\right)^{2} \mathrm{e}^{-(t-s)} \mathrm{d} x \mathrm{~d} s \\
& \leq 3 L \int_{\mathbb{R}^{d}}\left(f(x)-g_{n}(x)\right)^{2} \mathrm{~d} x \\
& \rightarrow 0 \quad \text { as } n \rightarrow \infty
\end{aligned}
$$

uniformly in $l$. By Lemma 4.5, the second term on the right-hand side of (4.9) is less than $3 t^{2} w_{d} b_{1}\left\|f-g_{n}\right\|(1 / d+1 / \delta) \int_{\mathbb{R}^{d}}\left|f(y)-g_{n}(y)\right| \mathrm{d} y$, which converges to 0 as $n \rightarrow \infty$ uniformly in $l$. By Lemma 4.3, the third term on the right-hand side of (4.9) is less than

$$
\begin{aligned}
\frac{3}{l^{d}} \mathrm{E}\left[\int_{\mathbb{R}^{d} \times[0, \infty)}\left(f-g_{n}\right)^{2}\left(\frac{x}{l}\right) \mathbf{1}_{[0, t]}(r) \hat{\eta}_{0}(\mathrm{~d} x, \mathrm{~d} r)\right] & \leq \frac{3}{l^{d}} \mathrm{E}\left[\int_{\mathbb{R}^{d}}\left(f-g_{n}\right)^{2}\left(\frac{x}{l}\right) \eta_{0}(\mathrm{~d} x)\right] \\
& =\frac{3 \mu_{1}}{l^{d}} \int_{\mathbb{R}^{d}}\left(f-g_{n}\right)^{2}\left(\frac{x}{l}\right) \mathrm{d} x \\
& =3 \mu_{1} \int_{\mathbb{R}^{d}}\left(f(x)-g_{n}(x)\right)^{2} \mathrm{~d} x \\
& \rightarrow 0 \quad \text { as } n \rightarrow \infty
\end{aligned}
$$

uniformly in $l$. Therefore, we have proved that the finite-dimensional distributions of $Y^{(l)}(f, \cdot)$ converge weakly to multivariate normal distributions. Since we can use similar arguments to prove the same result as Theorem 4.2 for spatial pure-birth processes, the finite-dimensional distributions of $U^{(l)}$ converge weakly to multivariate normal distributions. By Lemma 4.3, $\mathrm{e}^{t} H^{(l)}$ is an $\mathcal{F}_{t}$-martingale, we can obtain

$$
\mathrm{E}\left[\left(H_{t}^{(l)}\right)^{2}\right] \leq \mu_{1} \int_{\mathbb{R}^{d}} f^{2}(x) \mathrm{d} x .
$$

So $\left\{H_{t}^{(l)}: l \geq 1\right\}$ is relatively compact. Now we know that $\left\{H_{t}^{(l)}: l \geq 1\right\},\left\{U_{t}^{(l)}: l \geq 1\right\}$, and $\left\{Y^{(l)}(f, t): l \geq 1\right\}$ are relatively compact. Because $Y^{(l)}(f, t)=H_{t}^{(l)}+U_{t}^{(l)}-V_{t}^{(l)},\left\{V_{t}^{(l)}: l \geq\right.$ $1\}$ is relatively compact. Hence, $H^{(l)}, U^{(l)}$, and $V^{(l)}$ are relatively compact in $D_{\mathbb{R}}[0, \infty)$. For any sequence $\left\{l_{n}\right\}$ such that $l_{n} \rightarrow \infty$ as $n \rightarrow \infty$, the jump sizes of $H^{\left(l_{n}\right)}, U^{\left(l_{n}\right)}$, and $V^{\left(l_{n}\right)}$ are bounded by $\|f\| / l_{n}^{-d / 2} \rightarrow 0$; so all the limits of $H^{\left(l_{n}\right)}, U^{\left(l_{n}\right)}$, and $V^{\left(l_{n}\right)}$ have continuous paths. By Lemma 4.6, $\left\{Y^{\left(l_{n}\right)}(f, \cdot)\right\}$ is relatively compact and the limit of $\left\{Y^{\left(l_{n}\right)}(f, \cdot)\right\}$ has continuous paths. So $Y^{(l)}(f, \cdot)$ converges weakly to a continuous Gaussian process. By Lemma 4.6, $\left(Y^{(l)}\left(f_{1}, \cdot\right), \ldots, Y^{(l)}\left(f_{n}, \cdot\right)\right)$ converges weakly to a continuous Gaussian process. This completes the proof. 


\section{An example from spatial statistics}

Ripley (1979) addressed the simulation problem by developing a Markov chain Monte Carlo algorithm based on identifying a spatial birth and death process whose stationary distribution gives the desired spatial point process. Baddeley (2000) provided an analogous solution to the parameter estimation problem with the introduction of his time-invariance estimators.

Let $\left\{\pi_{\theta}, \theta \in \Theta\right\}$ be a parametric family of models. Typically, $\Theta$ is a subset of $\mathcal{R}^{k}$, but that is not necessary. Our problem is to estimate $\theta$ from a single observation drawn from $\pi_{\theta}$. For each $\theta$, let $\mathcal{A}_{\theta}$ be the generator of a Markov process for which $\pi_{\theta}$ is the unique stationary distribution. Typically, $\pi_{\theta}$ is characterized as the unique probability measure satisfying

$$
\int \mathscr{A}_{\theta} f \mathrm{~d} \pi_{\theta}=0 \text { for all } f \in \mathscr{D}\left(\mathscr{A}_{\theta}\right) \text {. }
$$

The domain $\mathscr{D}\left(\mathcal{A}_{\theta}\right)$ of $\mathscr{A}_{\theta}$ is usually a subset of the bounded measurable functions. For a onedimensional parameter $\theta$, we choose a function $f \in \bigcap_{\theta} \mathscr{D}\left(\mathcal{A}_{\theta}\right)$, and estimate $\theta$ by solving

$$
\mathcal{A}_{\theta} f(\eta)=0,
$$

where $\eta$ is the single observation drawn from $\pi_{\theta}$. Baddeley (2000) called (5.2) a time-invariance estimating equation, and called the solution of (5.2) a time-invariance estimator for $\theta$. Since (5.1) holds, (5.2) is an unbiased estimating equation.

In general, for a multidimensional parameter $\theta$, we choose a collection $f_{i}, i=1,2, \ldots, k$, where $f_{i} \in \cap_{\theta} \mathscr{D}\left(\mathcal{A}_{\theta}\right)$ and $k$ is the dimension of $\theta$. The estimator for $\theta$ then satisfies

$$
\mathcal{A}_{\theta} f_{i}(\eta)=0, \quad i=1, \ldots, k .
$$

We must choose $\left\{f_{i}, i=1,2, \ldots, k\right\}$ carefully to avoid inconsistencies among these $k$ equations.

For spatial point processes, the relationships between time-invariance and other methods of estimation (pseudo-likelihood and Takacs-Fiksel) have been discussed in Baddeley (2000). Baddeley gave some general discussion on the choice of the functions $f_{i}$; however, he did not provide any systematic discussion of the properties of particular classes of functions. Kurtz and $\mathrm{Li} \mathrm{(2003)} \mathrm{considered} \mathrm{the} \mathrm{following} \mathrm{two-parameter} \mathrm{families.}$

Nearest-neighbor interactions model. Let the parameter $\theta=\left(c_{1}, c_{2}\right)$, and let

$$
\lambda_{\theta}(x, \eta)=c_{1}+c_{2} \rho\left(\frac{\min _{x_{j} \in \eta}\left|x-x_{j}\right|}{t_{0}}\right) \text { and } b_{\theta}(x, \eta)=1
$$

be the birth rate and death rate of the spatial birth and death processes, respectively, where $c_{1}>0, c_{1}+c_{2} k>0, k$ is a positive constant, $\rho$ is a nonnegative function bounded by $k$, and $t_{0}$ is also a known positive constant. Suppose that $\eta$ has the stationary distribution of the spatial birth and death process. Kurtz and Li (2003) gave the conditions under which the stationary distribution was unique. They chose the functions $f_{1}$ and $f_{2}$ in the time-invariance estimation equations to be of the form

$$
f_{1}(\eta)=|\eta|, \quad f_{2}(\eta)=\frac{1}{|\eta|(|\eta|-1)} \sum_{\substack{i \neq j \\ i, j=1, \ldots,|\eta|}} \rho^{*}\left(\frac{\left|x_{i}-x_{j}\right|}{t_{0}}\right),
$$


where $|\eta|$ denotes the total variation of $\eta$ and $\rho^{*}$ is a measurable function. Then the timeinvariance estimators can be written as

$$
\begin{aligned}
& \hat{c}_{1}=g_{1}\left(Y_{1}, Y_{2}, Y_{3}, Y_{4}, Y_{5}\right):=\frac{\left(\left(Y_{1}-1 /|B|\right) Y_{4}-Y_{2} Y_{3}\right) Y_{1}}{\left(Y_{1}-1 /|B|\right)\left(Y_{4}-Y_{3} Y_{5}\right)}, \\
& \hat{c}_{2}=g_{2}\left(Y_{1}, Y_{2}, Y_{3}, Y_{4}, Y_{5}\right):=\frac{\left(Y_{1} Y_{2}-\left(Y_{1}-1 /|B|\right) Y_{5}\right) Y_{1}}{\left(Y_{1}-1 /|B|\right)\left(Y_{4}-Y_{3} Y_{5}\right)},
\end{aligned}
$$

where $B$ is the observation window and $|B|$ is its Lebesgue measure, and

$$
\begin{aligned}
Y_{1} & =\frac{|\eta|}{|B|}=\frac{\eta(B)}{|B|} \\
Y_{2} & =\frac{1}{|B|} \sum_{x \in \eta} \sum_{y \in \eta} \rho^{*}\left(\frac{|x-y|}{t_{0}}\right) \\
& =\frac{1}{|B|} \int_{B} \int_{B} \rho^{*}\left(\frac{|x-y|}{t_{0}}\right) \eta(\mathrm{d} x) \eta(\mathrm{d} y) \\
Y_{3} & =\frac{1}{|B|} \int_{B} \rho\left(\frac{\min _{x_{i} \in \eta}\left|u-x_{i}\right|}{t_{0}}\right) \mathrm{d} u, \\
Y_{4} & =\frac{1}{|B|} \int_{B} \sum_{j=1}^{|\eta|} \rho^{*}\left(\frac{\left|u-x_{j}\right|}{t_{0}}\right) \rho\left(\frac{\min _{x_{i} \in \eta}\left|u-x_{i}\right|}{t_{0}}\right) \mathrm{d} u \\
& =\frac{1}{|B|} \int_{B} \int_{B} \rho^{*}\left(\frac{|u-x|}{t_{0}}\right) \eta(\mathrm{d} x) \rho\left(\frac{\min _{x_{i} \in \eta}\left|u-x_{i}\right|}{t_{0}}\right) \mathrm{d} u \\
Y_{5} & =\frac{1}{|B|} \int_{B} \sum_{j=1}^{|\eta|} \rho^{*}\left(\frac{\left|u-x_{j}\right|}{t_{0}}\right) \mathrm{d} u=\int_{B} \int_{B} \rho^{*}\left(\frac{|u-x|}{t_{0}}\right) \eta(\mathrm{d} x) \mathrm{d} u .
\end{aligned}
$$

Typically this model does not have explicit forms for the density function. Consequently, it would be difficult to apply classical methods of estimation to these models. Kurtz and Li (2003) considered the following problem concerning the consistency of time-invariance estimators. Assume that the data comes by observing the point process in a finite subregion of an infinite region. The consistency question then becomes whether or not the parameter estimates converge to the correct value as data is collected from larger and larger subregions. They proved the consistency. The asymptotic normality is given in Qi (2007), where the ideas in this paper were used to prove the CLTs for the following functionals of $\eta$ :

$$
\eta(B), \quad \int_{B} \int_{B} \rho^{*}\left(\frac{|x-y|}{t_{0}}\right) \eta(\mathrm{d} x) \eta(\mathrm{d} y), \quad \int_{B} \rho\left(\frac{\min _{x_{i} \in \eta}\left|u-x_{i}\right|}{t_{0}}\right) \mathrm{d} u,
$$

and

$$
\int_{B} \int_{B} \rho^{*}\left(\frac{u-x}{t_{0}}\right) \eta(\mathrm{d} x) \rho\left(\frac{\min _{x_{i} \in \eta}\left|u-x_{i}\right|}{t_{0}}\right) \mathrm{d} u, \quad \int_{B} \int_{B} \rho^{*}\left(\frac{|u-x|}{t_{0}}\right) \eta(\mathrm{d} x) \mathrm{d} u .
$$

As the observation window $B$ goes to the whole space, then we can apply the delta method to give the asymptotic normality of the time-invariance estimators in (5.3). 


\section{Acknowledgements}

This paper is part of the author's $\mathrm{PhD}$ thesis written under the supervision of Professor Thomas G. Kurtz, whom the author would like to thank for suggesting the problem and helping to find its solution. I also thank the anonymous referees and the Editors for their stimulating remarks.

\section{References}

Baddeley, A. J. (2000). Time-invariance estimating equations. Bernoulli 6, 783-808.

Ethier, S. N. And Kurtz, T. G. (1985). Markov Processes: Characterization and Convergence. John Wiley, New York.

Garcia, N. L. And Kurtz, T. G. (2006). Spatial birth and death processes as solutions of stochastic equations. Alea 1, 281-303.

Holley, R. And Stroock, D. W. (1979). Central limit phenomena of various interacting systems. Ann. Math. 110, 333-393.

Kesten, H. And LeE, S. (1996). The central limit theorem for weighted minimal spanning trees on random points. Ann. Appl. Prob. 6, 495-527.

Kurtz, T. And Li, S. (2003). Time-invariance modeling and estimation for spatial point processes: general theory. Res. Rep., University of Wisconsin-Madison. Available at http://www.math.wisc.edu/ kurtz/papers/shun.pdf.

LeE, S. (1997). The central limit theorem for Euclidean minimal spanning trees. I. Ann. Appl. Probab. 7, 996-1020.

McLeish, D. L. (1974). Dependent central limit theorems and invariance principles. Ann. Prob. 2, 620-628.

Mitoma, I. (1983). Tightness of probabilities on c $\left([0,1] ; \psi^{\prime}\right)$ and $\mathrm{d}\left([0,1] ; \psi^{\prime}\right)$. Ann. Prob. 4, 989-999.

Penrose, M. D. (2001). A central limit theorem with applications to percolation, epidemics and Boolean models. Ann. Probab. 11, 1005-1041.

Penrose, M. D. (2005). Multivariate spatial central limit theorems with applications to percolation and spatial graphs. Ann. Prob. 33, 1945-1991.

Penrose, M. D. (2008). Existence and spatial limit theorems for lattice and continuum particle systems. Prob. Surveys 5, 1-36.

QI, X. (2007). The central limit theorems for space-time point processes. Doctoral Thesis, University of WisconsinMadison.

Revuz, D. AND Yor, M. (2004). Continuous Martingales and Brownian Motion, 3rd edn. Springer, Berlin.

RIPLEY, B. D. (1979). Algorithm AS 137: Simulating spatial patterns: dependent samples from a multivariate density. Appl. Statist. 28, 109-112. 